\title{
Um estudo sobre incompletude de geodésicas semi-Riemannianas
}

\author{
Lucas de Faccio Nunes
}

DisSERTAÇÃO APRESENTADA

$\mathrm{AO}$

Instituto DE MATEMÁtica E EstatísticA

DA

Universidade DE SÃo PAUlo

PARA

OBTENÇÃO DO TÍTULO

$\mathrm{DE}$

Mestre em CiênCIAS

Programa: Mestrado em Matemática

Orientador: Profa. Dra. Barbara Corominas Valério

São Paulo, agosto de 2019 


\section{Um estudo sobre incompletude de geodésicas semi-Riemannianas}

Esta versão da dissertação contém as correções e alterações sugeridas pela Comissão Julgadora durante a defesa da versão original do trabalho, realizada em 15/08/2019. Uma cópia da versão original está disponível no Instituto de Matemática e Estatística da Universidade de São Paulo.

Comissão Julgadora:

- Profa. Dra. Barbara Corominas Valério (orientadora) - IME-USP

- Prof. Dr. José Antonio Moraes Vilhena - UFPA

- Prof. Dr. Luiz Amancio Machado de Sousa Junior - UNIRIO 


\section{Agradecimentos}

Logo ao início da Ainulindalë, ao primeiro florescer frenético do canto, entre as fusas e terças menores, surgiu da voz o fundamental instrumento, a mais prolixa ferramenta, capaz de tudo descrever e enaltecer. Com ela tudo foi feito com precisão e maestria, sem que nada não pudesse ser explicado. E após eras de mundo consegui contemplar e apreciar parte diminuta dessa grande criação, vivenciar o pouco que meus olhos puderam enxergar e minha mente pôde compreender. O resultado desse vislumbre se apresenta nas páginas seguintes e nutro a humilde esperança de que elas possam um dia inspirar alguém a penetrar nessa ciência onde estruturas são perfeitas, curvas são suaves e resultados são primorosos.

E dentro de tão resoluto universo, não me é possível esquivar de mencionar a participação de alguns que suportaram minha jornada. Aos meus pais que, com um brilhantismo sem igual, me guiaram pelas mais nebulosas montanhas e confiaram em mim sem nunca pestanejarem, sendo ambos como o Sol para um andarilho perdido. Ao meu irmão que, com maestria única, sempre foi capaz de provocar ou acalmar o mar revolto que reside em mim, sendo ele como o Zéfiro para um navegante desavisado. Ao meu amigo que, com majoritária simplicidade, me inspirava a concluir essa jornada por dois, sendo ele como a Lua para um poeta confuso. À minha namorada que, com afeto ímpar, me mostrava minha força e me dava estímulo para seguir, sendo ela como o escudo de Aquiles para um soldado desarmado. E à minha vó que, mesmo relutando em aceitar sua importância, com suas histórias e relatos mais simples, sempre me inspirou a nunca fraquejar e nunca esperar que as coisas se resolvam sozinhas, sendo ela como o mar, a terra e o ar para um ser humano qualquer.

Mas nem mesmo a pessoa mais inspirada pode concluir algo sem saber o que fazer, então agradeço imensamente à minha orientadora por ter me mostrado onde começar, por onde deveria ir e aonde chegar. Sinto agora que sei um pouco mais sobre Matemática do que sabia quando entrei e devo isso à ela.

Uma parte da jornada é o fim e aqui atinjo o meu, quiçá aqui também seja o começo para um outro alguém.

Non omnis moriar multaque pars mei vitabit Libitinam

(Não morrerei de todo, e de mim a maior parte escapará a Libitina)

Horácio, Odes, III, 30. 


\section{Resumo}

NUNES, L. F. Um estudo sobre incompletude de geodésicas semi-Riemannianas. 2019. 97 f. Dissertação (Mestrado) - Instituto de Matemática e Estatística, Universidade de São Paulo, São Paulo, 2019.

Nesse trabalho apresentaremos alguns exemplos clássicos que evidenciam as diferenças entre a geometria Riemanniana e a semi-Riemanniana (Lorentziana) quanto à completude de geodésicas. Para isso, revisitaremos conceitos básicos de Geometria, seguido de uma introdução aos espaços vetoriais de Lorentz e um estudo inicial sobre o grupo de Lorentz $O_{1}(n)$. Nos capítulos finais discutiremos sobre completude de geodésicas e como se distanciam do caso Riemanniano.

Palavras-chave: Geodésica, Métricas de Lorentz, Completude de geodésicas. 


\section{Abstract}

NUNES, L. F. A study on uncompleteness of semi-Riemannian geodesics. 2019.

97 f. Dissertação (Mestrado) - Instituto de Matemática e Estatística, Universidade de São Paulo, São Paulo, 2019.

In this work we intend to present some classical examples that display the differences between Riemannian and semi-Riemannian (Lorentzian) geometry in relation to the completeness of geodesics. For this, we will revisit basic Geometry concepts followed by an introduction to the vector spaces of Lorentz and a simple study on the Lorentz group $O_{1}(n)$. In the final chapters we will discuss about the completeness of geodesics and how it distances itself from the Riemannian case.

Keywords: Geodesic, Lorentz metrics, Completeness of geodesics. 


\section{Sumário}

Lista de Figuras $\quad$ vi

1 Introdução 1

2 Conceitos preliminares $\quad 3$

2.1 Espaço dual e bidual . . . . . . . . . . . . . . . . . . 3

2.2 Tensores . . . . . . . . . . . . . . . . . . . . . . . . 4

2.2.1 Espaço de tensores . . . . . . . . . . . . . . . . . . . . 4

2.2.2 Produto Tensorial . . . . . . . . . . . . . . . . . . . 5

2.3 Formas bilineares simétricas e produtos escalares . . . . . . . . . . . . 6

2.4 Os isomorfismos flat e sharp . . . . . . . . . . . . . . . . 14

2.5 Derivada covariante . . . . . . . . . . . . . . . . 16

2.6 Derivada covariante ao longo de uma curva . . . . . . . . . . . . . . . . 25

2.7 Geodésicas . . . . . . . . . . . . . . . . . . 26

2.8 Aplicação exponencial . . . . . . . . . . . . . . . . . . . . . . . . . . 29

2.9 Campo de Killing . . . . . . . . . . . . . . . . . . . . . . . . . . . . . . 30

2.10 Relação entre formas bilineares e produtos escalares . . . . . . . . . . . . . 33

3 Espaços vetoriais de Lorentz 35

3.1 Cones tipo-tempo . . . . . . . . . . . . . . . . . . . . . . . 35

3.2 Desigualdades reversas . . . . . . . . . . . . . . . . . . 37

3.3 Cones causais . . . . . . . . . . . . . . . . . . . . . . . . 39

3.4 Subespaços Lorentzianos . . . . . . . . . . . . . . . . . . . . . . . . 40

3.5 Grupo de Lorentz . . . . . . . . . . . . . . . . . . . . . . . . . . . . . 42

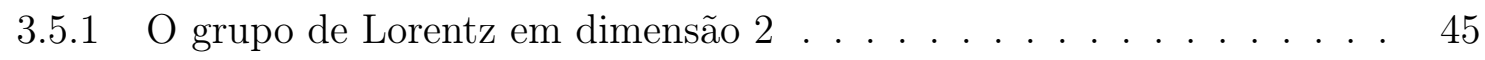

3.5.2 Grupos de Lorentz em dimensões maiores . . . . . . . . . . . . . . . 49

4 Geodésicas fechadas incompletas $\quad 57$

4.1 Um pouco sobre ações de grupo e espaço de órbitas . . . . . . . . . . . . . . . . . 57

4.2 Espaços homogêneos e geodésicas fechadas . . . . . . . . . . . . . . . . . 62 
5 Completude por caráter causal $\quad 69$

5.1 Métricas conformes e pré-geodésicas . . . . . . . . . . . . . . . . . . . . . . . . . . 69

5.2 Independência de caráter de completude . . . . . . . . . . . . 72

$\begin{array}{lll}6 & \text { Variedades compactas incompletas } & 78\end{array}$

6.1 Família de métricas com campo de Killing . . . . . . . . . . . . . . 78

6.2 O toro de Clifton-Pohl . . . . . . . . . . . . . . . . 84

$\begin{array}{ll}\text { Referências Bibliográficas } & 88\end{array}$ 


\section{Lista de Figuras}

2.1 Geodésica $\gamma$ com condições iniciais $\gamma(0)=p$ e $\gamma^{\prime}(0)=v \ldots$. . . . . . . . 27

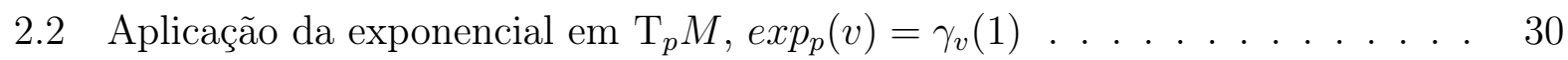

2.3 A exponecial é um difeomorfismo de $\tilde{U} \in \mathrm{T}_{p} M$ em $U \in M \ldots$. . . . . . . . . 30

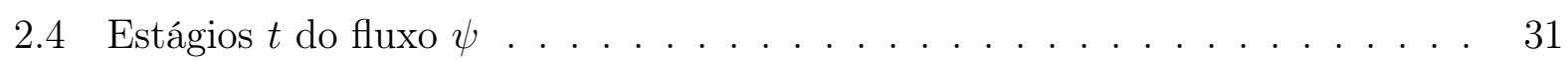

3.1 Os cones temporais em $\mathbb{L}^{3} \ldots \ldots \ldots \ldots$. . . . . . . . . . . . . . . . . . .

3.2 Representação da desigualdade triangular nos casos Euclidiano e não-Euclidiano 38

4.1 Representação de $\gamma \operatorname{com} \gamma^{\prime}(b)=-\gamma^{\prime}(a) \ldots \ldots$. . . . . . . . . . . 64

4.2 Representação "aberta" de $\gamma$ e reparametrização por $\alpha$ e $\beta$. . . . . . . . 65

4.3 Transformação de $C$ em $M$ a partir do grupo $G$ e relação de equivalência $\sim \quad 67$

4.4 Transformação de $\tilde{\gamma}$ em $\gamma$ pelo grupo $G$ e relação de equivalência $\sim$. . . . . 68

5.1 Local de definição dos vetores tangentes e representação da imagem da função $u 74$

5.2 Esboço aproximado de uma geodésica $\alpha \ldots \ldots$. . . . . . . . . . 75

5.3 Local de definição dos vetores tangentes e representação da imagem da função $u 76$

5.4 Esboço aproximado de uma geodésica $\alpha \ldots \ldots$. . . . . . . . . 76

6.1 O toro de Clifton-Pohl ainda sem a identificação das fronteiras . . . . . . . . 86 


\section{Capítulo 1}

\section{Introdução}

Os princípios da Geometria de Lorentz estão intimamente associados com o florescer das teorias da Relatividade. Os resultados iniciais de Einstein levaram à confirmação das transformações de Lorentz, que já faziam parte do grupo algébrico homônimo, chamado assim por Poincaré pouco tempo antes. De modo que o desenvolvimento referente à Relatividade e ao Grupo de Lorentz se suportaram mutuamente durante os anos. Entretanto, os constantes estudos por parte da comunidade matemática e o encontro dessa matéria com os conceitos já conhecidos das Geometrias não-Euclidianas levou ao desenvolvimento da Geometria semi-Riemanniana, em particular da Lorentziana. A partir de então essa disciplina conquistou seu próprio espaço dentro da Matemática e cresceu de modo independente à Física, embora seja mais uma das diversas pontes entre as duas áreas.

A principal referência desse trabalho foi [VC10], que serviu como um guia onde pudemos nos basear e, a partir dele, encontrar mais referências sobre o assunto. As influências desse livro podem ser observadas em toda a dissertação, embora sejam mais veementes no Capítulo 3. No desenrolar desse trabalho muitas referências se fizeram necessárias em cada um dos capítulos.

No Capítulo 2 estabelecemos alguns conceitos básicos e estruturamos os resultados mais essenciais de Geometria que utilizaremos no resto da dissertação. A maioria dos resultados valem independentes do caráter Riemanniano ou semi-Riemanniano da métrica considerada. É imprescindível destacar a importância de [O'N83], uma referência clássica quando em estudos de Geometria semi-Riemmaniana, porém também destacamos [Bie19] e [Pet06] que nos auxiliaram na construção dos resultados preliminares.

O capítulo 3 introduz os espaços vetorias de Lorentz que surgem ao se tomar um produto interno não necessariamente positivo definido. Trataremos um pouco sobre a base de um espaço com essa configuração e, consequentemente, sobre a matriz de transformações lineares nesses espaços. Veremos um pouco sobre os cones causais e alguns resultados pouco intuitivos quando comparados com suas versões Euclidianas. Terminaremos falando sobre o grupo de Lorentz, onde iremos construir todos os elementos de $O_{1}(2)$ e depois falaremos sobre a versão genérica $O_{1}(n)$.

Feito isso, teremos o que é necessário para estudarmos algumas questões sobre a incompletude de geodésicas. No capítulo 4 falaremos um pouco sobre ações de grupo e espaço de órbitas e como referência temos [KN63] e [Mun00]. Depois lidaremos com espaços homogêneos, quando evidenciaremos a invalidade do Teorema de Hopf-Rinow para variedades semi-Riemannianas e mostraremos uma versão mais adequada proveniente de [Mar73]. No final, construiremos uma variedade com geodésicas fechadas incompletas baseada em [CC08].

As variedades semi-Riemannianas permitem distinguir vetores pelo caráter causal, que 
será visto no capítulo 3, a mesma distinção será feita com geodésicas. No capítulo 5 falaremos sobre métricas conformes e pré-geodésicas e construiremos exemplos de métricas que podem ser completas e incompletas dependendo do caráter causal da geodésica. A referência principal foi [Ger68], porém [BEE96] auxiliou na construção do exemplo, sendo ambos baseados em [Kun63].

Encerraremos esse trabalho complementando uma discussão do capítulo 4 ao apresentarmos uma variedade compacta, mas não homogênea e, dessa forma, não completa. Faremos isso usando o toro $\mathrm{T}^{2}$ e a família de métricas construída em [Caj97], apresentando primeiro o caso genérico seguido de um caso particular. Seguindo [CR94], falaremos também do toro de Clifton-Pohl e geodésicas tipo-luz. 


\section{Capítulo 2}

\section{Conceitos preliminares}

\subsection{Espaço dual e bidual}

Nesse capítulo iremos apresentar alguns conceitos básicos que serão utilizados ao longo do trabalho, muitos desses são resultados essenciais em qualquer estudo de geometria e, como poderá ser observado, são independentes do tipo de métrica tomada, seja ela Riemanniana ou não-Riemanniana.

Definição 2.1. Seja $V$ um espaço vetorial real de dimensão finita. $O$ espaço dual $V^{*}$ consiste de funcionais lineares $\omega: V \rightarrow \mathbb{R}$. Se $\mathcal{B}=\left\{e_{1}, \ldots, e_{n}\right\}$ é uma base de $V$ então a base dual $\mathcal{B}^{*}=\left\{\varphi^{1}, \ldots, \varphi^{n}\right\}$ de $V^{*}$ é definida por $\varphi^{i}\left(e_{j}\right)=\delta_{i j}$, com $i, j=1, \ldots, n$. Logo,

$$
v=\sum_{i=1}^{n} \varphi^{i}(v) e_{i} \quad e \quad \omega=\sum_{j=1}^{n} \omega\left(e_{j}\right) \varphi^{j},
$$

para quaisquer $v \in V$ e $\omega \in V^{*}$.

Definição 2.2. Seja $V$ um espaço vetorial real de dimensão finita. O espaço dual $\left(V^{*}\right)^{*}$ do espaço dual de $V$ é chamado o espaço bidual de $V$ e denotado $V^{* *}$.

O Teorema a seguir propõe uma identificação natural entre $V$ e $V^{* *}$. Tal identificação será importante depois para entendermos Tensores.

Teorema 2.3. Seja $V$ um espaço vetorial com $\operatorname{dim} V=n$. Defina a aplicação $\Phi: V \rightarrow V^{* *}$ por

$$
v \mapsto \Phi(v)
$$

$\operatorname{com} \Phi(v): V^{*} \rightarrow \mathbb{R}$ tal que $\Phi(v)(\omega)=\omega(v)$. Então $\Phi$ é um isomorfismo natural entre $V e$ $V^{* *}$.

Demonstração. Vamos mostrar que $\Phi$ está bem definido. Sejam $v, w \in V$ tais que $v=w$, e seja $\omega \in V^{*}$, então

$$
\Phi(v)(\omega)=\omega(v)=\omega(w)=\Phi(w)(\omega)
$$

Temos ainda que $\Phi$ é linear. De fato, sejam $v, w \in V$ e $\alpha \in \mathbb{R}$ então para qualquer $\omega \in V^{*}$

$$
\Phi(\alpha v+\beta w)(\omega)=\omega(\alpha v+\beta w)=\alpha \omega(v)+\beta \omega(w)=\alpha \Phi(v)+\beta \Phi(w)
$$

Como $\operatorname{dim} V=\operatorname{dim} V^{* *}$, para verificar que $\Phi$ é um isomorfismo basta mostrar que ele é injetivo, isto é, que seu núcleo é o subespaço nulo. Seja $v \in V$ um vetor não nulo qualquer. 
Estenda este vetor a uma base $\mathcal{B}=\left\{v, e_{2} \ldots, e_{n}\right\}$ para $V$. Seja $\mathcal{B}^{*}=\left\{\varphi^{1}, \ldots, \varphi^{n}\right\}$ a correspondente base dual de $V^{*}$. Então $\Phi(v) \neq 0$ porque

$$
\Phi(v)\left(\varphi^{1}\right)=\varphi^{1}(v)=\delta_{11}=1 .
$$

Como todo elemento de $V$ é combinação linear dos elementos de $\mathcal{B}$ mostramos que $\Phi(w)=\dot{0}$, onde $\dot{0}$ é a função nula, somente se $w=0$. Portanto o núcleo tem dimensão nula e $\Phi$ é isomorfismo, pelo Teorema do Núcleo-Imagem.

\subsection{Tensores}

\subsubsection{Espaço de tensores}

Definição 2.4. Seja $V$ um espaço vetorial real de dimensão finita e $V^{*}$ seu espaço dual.

- Um r-tensor contravariante em $V$ (ou tensor contravariante de ordem r) é uma função $r$-linear

$$
T: \underbrace{V^{*} \times \ldots \times V^{*}}_{r \text { vezes }} \rightarrow \mathbb{R}
$$

$O$ conjunto dos r-tensores contravariantes em $V$ é denotado por $\mathcal{T}^{r}(V)$.

- Um s-tensor covariante em V (ou tensor covariante de ordem s) é uma função s-linear

$$
T: \underbrace{V \times \ldots \times V}_{s \text { vezes }} \rightarrow \mathbb{R}
$$

$O$ conjunto dos s-tensores covariantes em $V$ é denotado por $\mathcal{T}_{s}(V)$.

- Um tensor do tipo $(r, s)$ é um tensor r-contravariante e s-covariante, isto é, uma função real multilinear

$$
T: \underbrace{V^{*} \times \ldots \times V^{*}}_{r \text { vezes }} \times \underbrace{V \times \ldots \times V}_{s \text { vezes }} \rightarrow \mathbb{R}
$$

$O$ conjunto dos $(r, s)$-tensores sobre $V$ será denotado por $\mathcal{T}_{s}^{r}(V)$ e não é difícil ver que $\mathcal{T}_{s}^{r}(V)$ é um espaço vetorial.

Proposição 2.5. Definidas as operações de soma e multiplicação por escalar:

- Soma

$$
\begin{gathered}
+: \mathcal{T}_{s}^{r} \times \mathcal{T}_{s}^{r} \rightarrow \mathcal{T}_{s}^{r} \\
(T, S) \mapsto T+S,
\end{gathered}
$$

onde $(T+S)\left(\omega^{1}, \ldots, \omega^{r}, v_{1}, \ldots, v_{s}\right)=T\left(\omega^{1}, \ldots, \omega^{r}, v_{1}, \ldots, v_{s}\right)+S\left(\omega^{1}, \ldots, \omega^{r}, v_{1}, \ldots, v_{s}\right)$

- Multiplicação por escalar

$$
\begin{aligned}
& \cdot: \mathbb{R} \times \mathcal{T}_{s}^{r} \rightarrow \mathcal{T}_{s}^{r} \\
& (\lambda, T) \mapsto \lambda \cdot T,
\end{aligned}
$$

onde $(\lambda \cdot T)\left(\omega^{1}, \ldots, \omega^{r}, v_{1}, \ldots, v_{s}\right)=\lambda\left[T\left(\omega^{1}, \ldots, \omega^{r}, v_{1}, \ldots, v_{s}\right)\right]$.

Então o conjunto dos $(r, s)$-tensores, $\mathcal{T}_{s}^{r}$, juntamente com as operações de soma $e$ multiplicação por escalar é um espaço vetorial. 
Demonstração. Vamos mostrar que $\mathcal{T}_{s}^{r}$ é um espaço vetorial sobre $\mathbb{R}$. Definido o tensor $\dot{0} \in \mathcal{T}_{s}^{r}$, onde $\dot{0}\left(\omega^{1}, \ldots, \omega^{r}, v_{1}, \ldots, v_{s}\right)=0$, para todos $\omega^{1}, \ldots, \omega^{r} \in V^{*}, v_{1}, \ldots, v_{s} \in V$, como sendo o elemento neutro da soma o resultado segue facilmente do fato de $\mathbb{R}$ ser um espaço vetorial.

Exemplo 2.6. Podemos fazer algumas identificações naturais:

- Funcionais lineares em $V$ são 1-tensores covariantes: $\mathcal{T}_{1}(V)=V^{*}$;

- Usando a identificação do bidual vista no Teorema 2.3 podemos considerar que 1-tensores contravariantes são vetores de $V: \mathcal{T}^{1}(V)=V^{* *}=V$.

\subsubsection{Produto Tensorial}

Definição 2.7. Sejam $T$ e $S$ tensores do tipo $(r, s)$ e $(p, q)$, respectivamente. $O$ produto tensorial de $T$ e $S$ é o tensor $T \otimes S$ do tipo $(r+p, s+q)$ definido por

$(T \otimes S)\left(\omega^{1}, \ldots, \omega^{r+p}, v_{1}, \ldots, v_{s+q}\right)=T\left(\omega^{1}, \ldots, \omega^{r}, v_{1}, \ldots, v_{s}\right) . S\left(\omega^{r+1}, \ldots, \omega^{r+p}, v_{s+1}, \ldots, v_{s+q}\right)$.

No Teorema seguinte usaremos a identificação de $V$ com $V^{* *}$ para tratar os elementos $v \in V$ como sendo $v: V^{*} \rightarrow \mathbb{R}$.

Teorema 2.8. Se $\left\{e_{1}, \ldots, e_{n}\right\}$ é uma base para o espaço vetorial $V e\left\{\varphi^{1}, \ldots, \varphi^{n}\right\}$ é a correspondente base dual para $V^{*}$, então

$$
\mathcal{B}_{s}^{r}=\left\{e_{i_{1}} \otimes \ldots \otimes e_{i_{r}} \otimes \varphi^{j_{1}} \otimes \ldots \otimes \varphi^{j_{s}}\right\}_{\substack{1 \leq i_{1}, \ldots, i_{r} \leq n \\ 1 \leq j_{1}, \ldots, j_{s} \leq n}}
$$

é uma base para o espaço tensorial $\mathcal{T}_{s}^{r}(V)$. Além disso, qualquer tensor $T \in \mathcal{T}_{s}^{r}(V)$ se escreve na forma

$$
T=\sum_{\substack{i_{1}, \ldots, i_{r}=1 \\ j_{1}, \ldots, j_{s}=1}}^{n} T_{j_{1} \ldots j_{s}}^{i_{1} \ldots i_{r}} e_{i_{1}} \otimes \ldots \otimes e_{i_{r}} \otimes \varphi^{j_{1}} \otimes \ldots \otimes \varphi^{j_{s}}
$$

onde

$$
T_{j_{1} \ldots j_{s}}^{i_{1} \ldots i_{r}}=T\left(\varphi^{i_{1}}, \ldots, \varphi^{i_{r}}, e_{j_{1}}, \ldots, e_{j_{s}}\right) .
$$

Em particular, se $\operatorname{dim} V=n$ então $\operatorname{dim} \mathcal{T}_{s}^{r}=n^{r+s}$.

Demonstração. Primeiro mostraremos que $\mathcal{B}_{s}^{r}$ gera o espaço tensorial $\mathcal{T}_{s}^{r}(V)$. Seja $T \in \mathcal{T}_{s}^{r}(V)$ um tensor qualquer e defina

$$
T_{j_{1} \ldots j_{s}}^{i_{1} \ldots i_{r}}=T\left(\varphi^{i_{1}}, \ldots, \varphi^{i_{r}}, e_{j_{1}}, \ldots, e_{j_{s}}\right) .
$$

Se $v_{1}, \ldots, v_{s} \in V$ e $\omega^{1}, \ldots, \omega^{r} \in V^{*}$ são vetores arbitrários, expressos em coordenadas por

$$
v_{l}=\sum_{j_{l}=1}^{n} \alpha_{l}^{j_{l}} e_{j_{l}} \quad \text { e } \quad \omega^{k}=\sum_{i_{k}=1}^{n} \beta_{i_{k}}^{k} \varphi^{i_{k}}
$$

para $l=1, \ldots, s$ e $k=1, \ldots, r$, segue da multilinearidade que 


$$
\begin{aligned}
& T\left(\omega^{1}, \ldots, \omega^{r}, v_{1}, \ldots, v_{s}\right)=T\left(\sum_{i_{1}=1}^{n} \beta_{i_{1}}^{1} \varphi^{i_{1}}, \ldots, \sum_{i_{r}=1}^{n} \beta_{i_{r}}^{r} \varphi^{i_{r}}, \sum_{j_{1}=1}^{n} \alpha_{1}^{j_{1}} e_{j_{1}}, \ldots, \sum_{j_{s}=1}^{n} \alpha_{s}^{j_{s}} e_{j_{s}}\right) \\
& =\sum_{\substack{i_{1}, \ldots, i_{r}=1 \\
j_{1}, \ldots, j_{s}=1}}^{n} \alpha_{1}^{j_{1}} \ldots \alpha_{s}^{j_{s}} \cdot \beta_{i_{1}}^{1} \ldots \beta_{i_{r}}^{r} T\left(\varphi^{i_{1}}, \ldots, \varphi^{i_{r}}, e_{j_{1}}, \ldots, e_{j_{s}}\right) \\
& =\sum_{\substack{i_{1}, \ldots, i_{r}=1 \\
j_{1}, \ldots, j_{s}=1}}^{n} T_{j_{1} \ldots j_{s}}^{i_{1} \ldots i_{r}} \alpha_{1}^{j_{1}} \ldots \alpha_{s}^{j_{s}} \cdot \beta_{i_{1}}^{1} \ldots \beta_{i_{r}}^{r}=\sum_{\substack{i_{1}, \ldots, i_{r}=1 \\
j_{1}, \ldots, j_{s}=1}}^{n} T_{j_{1} \ldots j_{s}}^{i_{1} \ldots i_{r}} \varphi^{j_{1}}\left(v_{1}\right) \ldots \varphi^{j_{s}}\left(v_{s}\right) e_{i_{1}}\left(\omega^{1}\right) \ldots e_{i_{r}}\left(\omega^{r}\right) \\
& =\sum_{\substack{i_{1}, \ldots, i_{r}=1 \\
j_{1}, \ldots, j_{s}=1}}^{n} T_{j_{1} \ldots j_{s}}^{i_{1} \ldots i_{r}} e_{i_{1}} \otimes \ldots \otimes e_{i_{r}} \otimes \varphi^{j_{1}} \otimes \ldots \otimes \varphi^{j_{s}}\left(\omega^{1}, \ldots, \omega^{r}, v_{1}, \ldots, v_{s}\right) .
\end{aligned}
$$

Para mostrar que $\mathcal{B}_{s}^{r}$ é linearmente independente, suponha uma combinação linear nula

$$
T=\sum_{\substack{i_{1}, \ldots, i_{r}=1 \\ j_{1}, \ldots, j_{s}=1}}^{n} T_{j_{1} \ldots j_{s}}^{i_{1} \ldots i_{r}} e_{i_{1}} \otimes \cdots \otimes e_{i_{r}} \otimes \varphi^{j_{1}} \otimes \cdots \otimes \varphi^{j_{s}}=0
$$

Como

$$
\begin{aligned}
e_{i_{1}} \otimes \ldots \otimes & e_{i_{r}} \otimes \varphi^{j_{1}} \otimes \ldots \otimes \varphi^{j_{s}}\left(\varphi^{k_{1}}, \ldots, \varphi^{k_{r}}, e_{l_{1}}, \ldots, e_{l_{s}}\right) \\
& =e_{i_{1}}\left(\varphi^{k_{1}}\right) \ldots e_{i_{r}}\left(\varphi^{k_{r}}\right) \cdot \varphi^{j_{1}}\left(e_{l_{1}}\right) \ldots \varphi^{j_{s}}\left(e_{l_{s}}\right)=\delta_{k_{1} i_{1}} \ldots \delta_{k_{r} i_{r}} \delta_{j_{1} l_{1}} \ldots \delta_{j_{s} l_{s}}
\end{aligned}
$$

segue que tomando $k_{1}=i_{1}, \ldots, k_{r}=i_{r}$ e $l_{1}=j_{1}, \ldots, l_{s}=j_{s}$ teremos

$$
0=T\left(\varphi^{i_{1}}, \ldots, \varphi^{i_{r}}, e_{j_{1}}, \ldots, e_{j_{s}}\right)=T_{j_{1} \ldots j_{s}}^{i_{1} \ldots i_{r}}
$$

para todos os índices $i_{1}, \cdots, i_{r}, j_{1}, \cdots, j_{s}=1, \cdots, n$. Portanto, $\mathcal{B}_{s}^{r}$ é linearmente independente e base para $\mathcal{T}_{s}^{r}$.

\subsection{Formas bilineares simétricas e produtos escalares}

Definição 2.9. Seja $V$ um espaço vetorial de dimensão finita. Definimos uma forma bilinear de $V$ como sendo um elemento $b \in \mathcal{T}_{2}(V)$, ou seja, $b: V \times V \rightarrow \mathbb{R}$ linear em ambas as coordenadas.

Definição 2.10. Seja $b: V \times V \rightarrow \mathbb{R}$ uma forma bilinear. Dizemos que $b$ é simétrica se, para quaisquer $v, w \in V, b(v, w)=b(w, v)$.

Definição 2.11. Sejam $V$ um espaço vetorial de dimensão finita e $b: V \times V \rightarrow \mathbb{R}$ uma forma bilinear simétrica. Diremos que bé:

i. positiva definida (respectivamente negativa definida), se $b(v, v)>0$ (respectivamente $b(v, v)<0)$ para todo $v \in V-\{0\}$;

ii. positiva semidefinida (respectivemante negativa semidefinida), se $b(v, v) \geq 0$ (respectivamente $b(v, v) \leq 0$ ) para todo $v \in V-\{0\}$; 
iii. indefinida, se não é positiva semidefinida nem negativa semidefinida;

Definição 2.12. Sejam $V$ um espaço vetorial de dimensão finita e $b: V \times V \rightarrow \mathbb{R}$ uma forma bilinear simétrica. Definimos $o$ radical de $b$ como sendo o conjunto $N=\{v \in V \mid b(v, w)=0, \forall w \in V\}$.

Dizemos que b é não-degenerada, se $N=\{0\}$. Caso contrário, se $N \neq\{0\}, b$ é dito degenerado.

Definição 2.13. Seja $b: V \times V \rightarrow \mathbb{R}$ uma forma bilinear simétrica. Definimos $q_{b}: V \rightarrow \mathbb{R}$ uma forma quadrática associada a $b$ dada por $q_{b}(v)=b(v, v)$.

Dada uma forma quadrática $q_{b}$ é possível escrever $b$ a partir de $q_{b}$ da seguinte forma

$$
b(v, w)=\frac{1}{2}\left[q_{b}(v+w)-q_{b}(v)-q_{b}(w)\right] .
$$

De fato,

$$
\begin{aligned}
& \frac{1}{2}\left[q_{b}(v+w)-q_{b}(v)-q_{b}(w)\right]=\frac{1}{2}[b(v+w, v+w)-b(v, v)-b(w, w)] \\
& \quad=\frac{1}{2}[b(v, v)+b(v, w)+b(w, v)+b(w, w)-b(v, v)-b(w, w)]=\frac{1}{2}[2 b(v, w)]=b(v, w) .
\end{aligned}
$$

Definição 2.14. Sejam $(V, b)$ como acima e $v \in V$ com $v \neq 0$, diremos que $v$ é:

i. tipo-tempo, se $q_{b}(v)<0$;

ii. tipo-luz, se $q_{b}(v)=0$;

iii. tipo-espaço, se $q_{b}(v)>0$.

Note que a definição acima estabelece uma relação semelhante a tricotomia, as denominações estabelecidas serão utilizadas durante todo o texto e perceba que estão associadas diretamente com a forma bilinear simétrica considerada.

Podemos definir a norma de um vetor $v$ como $\|v\|=\sqrt{\left|q_{b}(v)\right|}$.

Definição 2.15. Um produto escalar $g$ em $V$ é uma forma bilinear simétrica nãodegenerada.

Por vezes, utilizaremos a notação $\langle\cdot, \cdot\rangle$ para denotar $g$.

Essa definição nos distancia da ideia de produto escalar euclidiano, de fato, tal definição estende o conceito de produto escalar já que não há a necessidade de $g$ ser positiva definida, como é esperado quando consideramos situações euclidianas.

Definição 2.16. Seja $(V, g)$ um espaço vetorial munido de produto escalar $g$. Se $v, w \in V$ dizemos que $v$ é ortogonal a $w$ se $g(v, w)=0$. Consequentemente dizemos que $A, B \subseteq V$ são ortogonais se $v$ é ortogonal a $w$ para quaisquer $v \in A$ e $w \in B$. Denotamos o conjunto dos vetores ortogonais a $A$ por

$$
A^{\perp}=\{v \in V \mid g(v, w)=0, \forall w \in A\}
$$

Além disso, se $\operatorname{dim} V=n<\infty$, seja $\left\{e_{1}, \ldots, e_{n}\right\}$ uma base de $V$. Tal base é dita ortonormal se cada elemento tem norma 1 , ou seja, $\left\|e_{i}\right\|=\sqrt{\left|g\left(e_{i}, e_{i}\right)\right|}=1$, para todo $i=1, \ldots, n$ e se são dois a dois ortogonais, ou seja, $g\left(e_{i}, e_{j}\right)=0$, para $i \neq j$. 
É importane notar que não existem vetores do tipo-luz numa base ortonormal, já que se $v \in V$ é tal que $b(v, v)=0$ então $\|v\|=0$.

Exemplo 2.17. Considere $g: \mathbb{R}^{2} \times \mathbb{R}^{2} \rightarrow \mathbb{R}$, definido por $g(v, w)=v_{1} w_{1}-v_{2} w_{2}$, onde $v=\left(v_{1}, v_{2}\right)$ e $w=\left(w_{1}, w_{2}\right)$.

Claramente, $g$ é simétrica e bilinear. Também é não-degenerada, de fato, suponha que exista $v \in V$ tal que $g(v, w)=0$, para qualquer $w \in V$. Considere $w_{1}=(1,0)$ e $w_{2}=(0,1)$, então

$$
\left\{\begin{array}{l}
0=g(v,(1,0))=v_{1} \\
0=g(v,(0,1))=-v_{2}
\end{array}\right.
$$

Logo, $v=(0,0)$. Portanto, $g$ é produto escalar.

Veja que a forma quadrática associada é $q_{g}(v)=v_{1}^{2}-v_{2}^{2}$, logo g é uma forma bilinear indefinida, pois se $v_{1}>v_{2}$ então $g(v, v)>0$ e, caso contrário, $g(v, v)<0$.

Considere agora os seguintes vetores $(1, y)$ e $(y, 1)$, para qualquer $y \in \mathbb{R}$, veja que $g((1, y),(y, 1))=0$. Logo, $(1, y)$ e $(y, 1)$ são ortogonais entre si, mostrando uma forte diferença com a noção de ortogonalidade euclidiana.

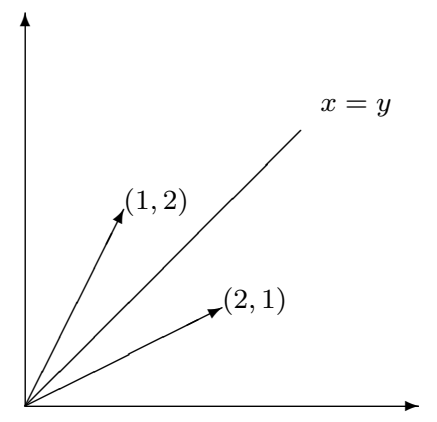

Se tomarmos $y=2$ podemos ver que $(1,2)$ e $(2,1)$ não são ortogonais no sentido euclidiano. Note que $(1,1)$ é ortogonal à si próprio, já que é tipo-luz, assim como todo vetor do tipo $(x, x)$.

Como vimos no exemplo acima, um produto escalar com sinal negativo tem um forte impacto nas relações entre os vetores provenientes do produto. A classificação de vetores vista na Definição 2.14 generaliza muitos outros conceitos, a própria ideia de ortogonalidade entre vetores ganha novas interpretações. Vamos agora juntar essas duas novas visões para conceitos conhecidos para estudar as bases de um espaço com produto escalar semelhante ao exemplo acima e ver suas diferenças e semelhanças. Iremos considerar sempre um espaço vetorial $V$ de dimensão finita $n$.

Definição 2.18. Sejam $V$ um espaço vetorial e $g$ um produto escalar. Definimos o índice de $(V, g)$, denotado por $\nu$, como sendo o número de vetores tipo-tempo numa base ortonormal de $V$.

Equivalentemente, o índice $\nu$ de uma forma bilinear simétrica $b$ em $V$ é o maior número inteiro igual a dimensão de um subespaço $W \subset V$ onde $\left.b\right|_{W}$ é negativa definida.

O resultado a seguir mostra a independência do índice em relação a escolha da base de $(V, g)$

Lema 2.19. Dada uma base ortonormal $\mathcal{B}$ de $(V, g)$, o índice $\nu$ não depende da escolha da base, mas de $(V, g)$.

Demonstração. Suponha que $\operatorname{dim} V=n$ e considere a base ortonormal $\mathcal{B}=\left\{e_{1}, \ldots, e_{k}, f_{1}, \ldots, f_{\nu}\right\}$ sendo $f_{j}$ vetores tipo-tempo e $e_{i}$ vetores tipo-espaço, com 
$\nu+k=n$. Suponha que exista uma outra base ortonormal de $V, \mathcal{B}^{\prime}=\left\{u_{1}, \ldots, u_{l}, x_{1}, \ldots, x_{\nu^{\prime}}\right\}$ com $l+\nu^{\prime}=n$, e suponha, sem perda de generalidade, que $\nu^{\prime}>\nu$.

Considere os subespaços $X=\operatorname{span}\left(x_{1}, \ldots, x_{\nu^{\prime}}\right)$ e $W=\operatorname{span}\left(e_{1}, \ldots, e_{k}\right)$, note que a dimensão de $X \cap W$ deve ser maior que zero, caso contrário, $\operatorname{dim}(X+W)=\nu^{\prime}+k>\nu+k=n$, que é um absurdo.

Como $\operatorname{dim}(X \cap W)>0$, existe $u \in X \cap W$ com $u \neq 0$ tal que $g(u, u)>0$ e $g(u, u)<0$. O que é um absurdo, logo segue o resultado.

Definição 2.20. Seja $(V, g)$ um espaço vetorial com produto escalar. Um subespaço vetorial $W<V$ é dito não-degenerado se $W \cap W^{\perp}=\{0\}$ (ou, equivalentemente, se a restrição $g_{W}=\left.g\right|_{W \times W}$ é não-degenerada).

Proposição 2.21. Se $W<V$ e g é não-degenerado então:

i. $\operatorname{dim} W+\operatorname{dim} W^{\perp}=\operatorname{dim} V$;

ii. $\left(W^{\perp}\right)^{\perp}=W$;

iii. $V=W+W^{\perp} \Leftrightarrow W$ é não-degenerado $\left(\Leftrightarrow W^{\perp}\right.$ é não-degenerado $)$.

Demonstração. $\quad i$. Seja $e_{1}, \ldots, e_{\rho}, e_{\rho+1}, \ldots, e_{n}$ uma base de $V$ tal que $e_{1}, \ldots, e_{\rho}$ é base de $W$. Se $v=\sum_{i=1}^{n} a^{i} e_{i}$, então

$$
v \in W^{\perp} \Leftrightarrow g\left(v, e_{i}\right)=0, \forall i \in\{1, \ldots, \rho\} \Leftrightarrow \sum_{j=1}^{n} g_{i j} a^{j}=0, \forall i \in\{1, \ldots, \rho\},
$$

onde $g_{i j}=g\left(e_{i}, e_{j}\right)$. Assim, obtemos o seguinte sistema

$$
\left\{\begin{array}{c}
g_{11} a^{1}+g_{12} a^{2}+\ldots+g_{1 n} a^{n}=0 \\
\vdots \\
g_{\rho 1} a^{1}+g_{\rho 2} a^{2}+\ldots+g_{\rho n} a^{n}=0
\end{array}\right.
$$

que possui $\rho$ equações independentes e $n$ variáveis. E como $g$ é não-degenerado então a matriz $\rho \times n$ dos coeficiente do sistema

$$
\left[\begin{array}{ccccc}
g_{11} & \cdots & g_{1 \rho} & \cdots & g_{1 n} \\
\vdots & \ddots & \vdots & \ddots & \vdots \\
g_{\rho 1} & \cdots & g_{\rho \rho} & \cdots & g_{\rho n}
\end{array}\right]
$$

tem posto máximo $\rho$. Portanto, $\operatorname{dim} W^{\perp}=n-\rho$.

ii. Temos que $\left(W^{\perp}\right)^{\perp}=\left\{v \in V \mid g(v, w)=0, \forall w \in W^{\perp}\right\}$, logo é fácil ver que $W \subset\left(W^{\perp}\right)^{\perp}$. Aplicando o item 1. para $W^{\perp}$ teremos que $\operatorname{dim} W^{\perp}+\operatorname{dim}\left(W^{\perp}\right)^{\perp}=\operatorname{dim} V$, então $\operatorname{dim} W=\operatorname{dim}\left(W^{\perp}\right)^{\perp}$ e vale a igualdade.

iii. $(\Rightarrow)$ Pela hipótese, temos $\operatorname{dim} V=\operatorname{dim} W+\operatorname{dim} W^{\perp}-\operatorname{dim} W \cap W^{\perp}$ e pelo item 1 . temos $\operatorname{dim} V=\operatorname{dim} W+\operatorname{dim} W^{\perp}$, portanto $\operatorname{dim} W \cap W^{\perp}=0 . \operatorname{Logo}, W \cap W^{\perp}=0$ e portanto $W$ é não-degenerado.

$(\Leftarrow)$ Segue imediatamente da definição de não-degenerado e do item 1 . 
A segunda equivalência é análoga, usando também o item 2.

A Proposição 2.21 mostra que se um subespaço $W$ é não-degenerado então $V=W \oplus W^{\perp}$.

Teorema 2.22. Sejam $V$ um espaço vetorial de dimensão finita n e $g$ um produto escalar em $V$. Então $(V, g)$ admite base ortonormal.

Demonstração. Vamos provar por indução sobre a dimensão de $V$. Suponha que $\operatorname{dim} V=1$, como $g$ é não-degenerado, então existe $v \in V$ tal que $g(v, v) \neq 0$. Logo, $u=\frac{v}{\|v\|}$ é uma base ortonormal.

Assuma que $\operatorname{dim} V=n$ e que o resultado vale para $k<n$. De novo, como $g$ é não-degenerado, existe $v \in V$ tal que $g(v, v) \neq 0$. Da Proposição 2.21, itens $i$. e iii., $(\operatorname{span}(v))^{\perp}$ tem dimensão $n-1$ e é não-degenerado. Pela hipótese de indução existe $e_{1}, \ldots, e_{n-1}$, base ortonormal de $(\operatorname{span}(v))^{\perp}$. Portanto, $\left\{e_{1}, \ldots, e_{n-1}, \frac{v}{\|v\|}\right\}$ é base ortonormal de $V$.

Corolário 2.23. Toda base ortonormal de um subespaço não-degenerado $U$ de $V$ pode ser estendida a uma base ortonormal de $(V, g)$.

Demonstração. Como $U$ é não-degenerado, então $U^{\perp}$ também o é, pela Proposição 2.21 . Logo, $U^{\perp}$ tem base ortonormal e basta tomar a união das bases de $U$ e $U^{\perp}$.

Definição 2.24. Dizemos que um produto escalar $g$ é:

i. Euclidiano ou Riemanniano, se $\nu=0$;

ii. Lorentziano, se $\nu=1$ e $n \geq 2$;

iii. Semi-Euclidiano ou Semi-Riemanniano, se $\nu \geq 1$ e $n \geq 2$.

Exemplo 2.25. Em $\mathbb{R}^{n}$ podemos definir o produto escalar usual de indice $\nu$, denotado $\operatorname{por}\langle\cdot, \cdot\rangle_{\nu}$, como

$$
\left\langle\left(a_{1}, \ldots, a_{n}\right),\left(b_{1}, \ldots, b_{n}\right)\right\rangle_{\nu}=\sum_{i=1}^{n-\nu} a_{i} b_{i}-\sum_{i=n-\nu+1}^{n} a_{i} b_{i} .
$$

Note que se $n=2$ e $\nu=1$, obteremos o produto escalar Lorentziano do Exemplo 2.17:

$$
\left\langle\left(a_{1}, a_{2}\right),\left(b_{1}, b_{2}\right)\right\rangle_{1}=a_{1} b_{1}-a_{2} b_{2} .
$$

Definição 2.26. Sejam $(V, g)$ um espaço vetorial munido de um produto escalar $g$ e $\mathcal{B}$ uma base qualquer de $V$. Definimos por

i. $\pi$, o número de vetores do tipo-espaço em $\mathcal{B}$;

ii. $\nu$, como definido anteriormente, o número de vetores tipo-tempo em $\mathcal{B}$;

iii. $\mu$, o número de vetores do tipo-luz em $\mathcal{B}$.

Definição 2.27. Sejam $V$ um espaço de dimensão finita e $\mathcal{B}$ uma base de $V$. Dizemos $q u e \mathcal{B}$ é uma base de Sylvester se seus elementos são dois a dois ortogonais e, além disso, todos os elementos são unitários ou tipo-luz. 
Teorema 2.28 (Lei da Inércia de Sylvester). Se b é uma forma bilinear simétrica em $V$, então existe uma base de Sylvester para $V$.

Em particular, o número de vetores do tipo-espaço, tipo-tempo e tipo-luz não dependem da escolha da base.

Demonstração. Vamos mostrar que o radical de $b, N=\{v \in V \mid b(v, w)=0, \forall w \in V\}$ é um subespaço de $V$. De fato, $0 \in N$ e se $v, w \in N$, então, para qualquer $u \in V$,

$$
b(\alpha v+\beta w, u)=\alpha b(v, u)+\beta b(w, u)=0,
$$

$\operatorname{logo} \alpha v+\beta w \in N$ e $N$ é subespaço de $V$.

Defina, a partir de $b$, a seguinte forma bilinear simétrica $\bar{b}: V / N \times V / N \rightarrow \mathbb{R}$ dada por $\bar{b}([u],[v])=b(u, v)$. Note que $\bar{b}$ é não-degenerada. De fato, suponha que exista $[w] \in V / N$ tal que $\bar{b}([w],[v])=0$, para qualquer $[v] \in V / N$. Por definição, $0=\bar{b}([w],[v])=b(w, v)$, para qualquer $v \in V$, já que $\pi: V \rightarrow V / N$ é sobrejetora. Assim, $w \in N$ e $[w]=[0]$.

Agora temos que $\bar{b}$ é um produto escalar e pelo Teorema 2.22, $(V / N, \bar{b})$ admite uma base ortonormal $\overline{\mathcal{B}}=\left\{\left[e_{1}\right], \ldots,\left[e_{p}\right]\right\}$. Escolha uma base $\left\{e_{p+1}, \ldots, e_{p+\mu}\right\}$ para $N$. Portanto, $\left\{e_{1}, \ldots, e_{p}, e_{p+1}, \ldots, e_{p+\mu}\right\}$ é a base de $V$ desejada.

A Lei de Sylvester nos fornece uma base com as características mais favoráveis possíveis quando consideramos formas bilineares simétricas genéricas. Tal base propõe e estimula um tratamento matricial. A matriz de um forma bilinear é facilmente construída, seja $\mathcal{B}=\left\{e_{1}, \ldots, e_{n}\right\}$ uma base de $V$, a matriz $n \times n$ dada por $\left(b_{i j}\right)=b\left(e_{i}, e_{j}\right)$ é chamada de matriz de $b$ relativa a base $\mathcal{B}$, denotado por $[b]_{\mathcal{B}}$. Como $b$ é simétrica então $\bar{b}$ também o é.

A Lei de Sylvester pode ser vista numa versão matricial.

Teorema 2.29 (Outra versão da Lei de Sylvester). Suponha $V$ um espaço vetorial real e $b$ uma forma bilinear simétrica em $V$. Então existe uma base ordenada $\mathcal{B}$ de $V$ tal que a matriz de representação de $b$ nessa base é da forma $I_{\pi} \oplus-I_{\nu} \oplus 0_{\mu}$. Além disso, $\pi, \nu, \mu$ não dependem da escolha da base, mas somente de $b$.

Para entender esse resultado e suas consequências vamos partir do Teorema 2.28. Dado que $\mathcal{B}=\left\{e_{1}, \ldots, e_{p}, e_{p+1}, \ldots, e_{p+\mu}\right\}$ é uma base de Sylvester para $V$ então entre os vetores $e_{1}, \ldots, e_{p}$ podemos ter vetores tipo-espaço e tipo-tempo, assim podemos reescrevê-los da seguinte forma $e_{1}, \ldots, e_{\pi}, \ldots, e_{\pi+1}, \ldots, e_{\pi+\nu}$, distinguindo-os entre os de tipo-espaço e tipotempo.

Como $\mathcal{B}$ é ortogonal então a matriz de $b$ só terá elementos diferentes de zero na diagonal principal e terá a seguinte configuração:

$$
[b]_{\mathcal{B}}=\left[\begin{array}{ccc}
{[b]_{\pi}} & \cdots & 0 \\
\vdots & {[b]_{\nu}} & \vdots \\
0 & \cdots & {[b]_{\mu}}
\end{array}\right]
$$

sendo $[b]_{\pi},[b]_{\nu}$ e $[b]_{\mu}$ as submatrizes de $b$ referentes aos subespaços gerados pelos vetores tipo-espaço, tipo-tempo e tipo-luz.

Os vetores $e_{1}, \ldots, e_{\pi}$ são unitários e tipo-espaço, então $b\left(e_{i}, e_{i}\right)=1$. Os vetores $e_{\pi+1}, \ldots, e_{\pi+\nu}$ são tipo-tempo, então $b\left(e_{j}, e_{j}\right)=-1$. Os vetores $e_{p+1}, \ldots, e_{p+\mu}$ são tipo-luz, $\operatorname{logo} b\left(e_{k}, e_{k}\right)=0$. Portanto, 


$$
[b]_{\mathcal{B}}=\left[\begin{array}{ccccccccc}
1 & \cdots & 0 & 0 & \cdots & . & . & \cdots & 0 \\
\vdots & \ddots & \vdots & 0 & \cdots & . & . & \cdots & 0 \\
0 & \cdots & 1 & 0 & \cdots & . & . & \cdots & 0 \\
0 & \cdots & 0 & -1 & \cdots & 0 & 0 & \cdots & 0 \\
0 & \cdots & 0 & \vdots & \ddots & \vdots & 0 & \cdots & 0 \\
0 & \cdots & 0 & 0 & \cdots & -1 & 0 & \cdots & 0 \\
0 & \cdots & . & . & \cdots & 0 & 0 & \cdots & 0 \\
0 & \cdots & . & . & \cdots & 0 & \vdots & \ddots & \vdots \\
0 & \cdots & . & . & \cdots & 0 & 0 & \cdots & 0
\end{array}\right] .
$$

Tal matriz é exatamente a matriz proposta no Teorema 2.29. De modo equivalente, a própria estrutura da matriz, como mostrado no Teorema 2.29, nos leva para um base descrita no Teorema 2.28 .

Observação 2.30. Sejam $V$ um espaço de dimensão finita $n \quad e$ $\mathcal{B}=\left\{e_{1}, \ldots, e_{\pi}, f_{1}, \ldots, f_{\nu}, g_{1}, \ldots, g_{\mu}\right\}$ uma base de Sylvester.

1. O subespaço gerado pelos $\mu$ vetores tipo-luz de qualquer base de Sylvester coincide com o radical $N$ de $b$ e, além disso, não depende da escolha da base.

Demonstração. Considere $\operatorname{span}\left(g_{1}, \ldots, g_{\mu}\right)$. Seja $v \in N \subset V$, isto é, $b(v, w)=0$, para todo $w \in V$. Como

$$
v=\sum_{i=1}^{\pi} \alpha^{i} e_{i}+\sum_{j=1}^{\nu} \beta^{j} f_{j}+\sum_{k=1}^{\mu} \gamma^{k} g_{k}
$$

segue que

$$
0=b\left(v, e_{i}\right)=\alpha^{i} \quad \text { e } \quad 0=b\left(v, f_{j}\right)=\beta^{j},
$$

para quaisquer $i, j=1, \ldots, n$. Logo,

$$
v=\sum_{k=1}^{\mu} \gamma^{k} g_{k}
$$

ou seja $v \in \operatorname{span}\left(g_{1}, \ldots, g_{\mu}\right)$, donde segue que $N \subset \operatorname{span}\left(g_{1}, \ldots, g_{\mu}\right)$. Consideremos agora $v \in \operatorname{span}\left(g_{1}, \ldots, g_{\mu}\right)$ com $v \neq 0$. Seja $w \in V$, qualquer,

$$
w=\sum_{i=1}^{\pi} \alpha^{i} e_{i}+\sum_{j=1}^{\nu} \beta^{j} f_{j}+\sum_{k=1}^{\mu} \gamma^{k} g_{k} .
$$

Então,

$$
b(v, w)=b\left(\sum_{k=1}^{\mu} \delta^{k} g_{k}, w\right)=0
$$

pois $\mathcal{B}$ é ortogonal. Portanto, $N=\operatorname{span}\left(g_{1}, \ldots, g_{\mu}\right)$.

2. Seja $W<V$, a restrição $b_{W}$ de $b$ é uma nova forma bilinear em $W$ que pode ser degenerada mesmo que b não seja. É fácil ver que uma base ortogonal de $W$ com todos os vetores unitários ou do tipo-luz pode ser estendido a uma base de $V$ com as mesmas propriedades se, e só se, o radical $N_{W}$ de $b_{W}$ está contido no radical $N$ de $b$. 
Demonstração. Considere o produto escalar em $\mathbb{R}^{2}$ visto no Exemplo 2.17 $\left\langle(x, t),\left(x^{\prime}, t^{\prime}\right)\right\rangle_{1}=x x^{\prime}-t t^{\prime}$. Considere $W=\operatorname{span}(1,1), W<\mathbb{R}^{2}$. A restrição de $\langle\cdot, \cdot\rangle_{1}$ à $W$ é degenerada. Sejam $(1,1) \in W$ e $(x, x)$ um vetor qualquer de $W$.

$$
\langle(1,1),(x, x)\rangle_{1}=x-x=0 .
$$

$(\Rightarrow)$ Óbvio, pois os radicais são gerados pelos vetores tipo-luz das bases de Sylvester.

$(\Leftarrow)$ Por hipótese, $N_{W} \subset N$ e ainda $W<V$. Portanto, $\frac{W}{N_{W}} \subset \frac{V}{N}$.

3. Sejam $V$ e $V^{\prime}$ espaços vetoriais e $b$ e $b^{\prime}$ formas bilineares associadas a $V$ e $V^{\prime}$. Existe um isomorfismo $\Psi: V \rightarrow V^{\prime}$ que preserva $b$ e $b^{\prime}\left(b(x, y)=b^{\prime}(\Psi(x), \Psi(y))\right)$ se, e só se, $n=n^{\prime}, \mu=\mu^{\prime}$ e $\nu=\nu^{\prime}$.

Demonstração. $(\Leftarrow)$ Basta tomar $\Psi: V \rightarrow V^{\prime}$ tal que $\Psi\left(e_{i}\right)=e_{i}^{\prime}, \Psi\left(f_{j}\right)=f_{j}^{\prime}$ e $\Psi\left(g_{k}\right)=g_{k}^{\prime}$.

$(\Rightarrow)$ Sabendo que existe $\Psi: V \rightarrow V^{\prime}$ isomorfismo então temos que $n=n^{\prime}$. Note que os radicias $N_{b}$ e $N_{b^{\prime}}$ são tais que $\Psi\left(N_{b}\right)=N_{b^{\prime}}$.

$$
\begin{gathered}
x \in N_{b} \Rightarrow b(x, w)=0, \forall w \in V \Rightarrow b^{\prime}(\Psi(x), \Psi(w))=0, \forall w \in V \Rightarrow \Psi(x) \in N_{b^{\prime}} . \\
y \in N_{b^{\prime}} \Rightarrow b^{\prime}(y, w)=0, \forall w \in V^{\prime} \Rightarrow 0=b^{\prime}(\Psi(x), \Psi(v))=b(x, v), \forall v \in V \Rightarrow x \in N_{b}
\end{gathered}
$$

Como $\Psi$ é isomorfismo então $N_{b} \approx N_{b^{\prime}}$, portanto $\mu=\mu^{\prime}$ e $\frac{V}{N_{b}} \approx \frac{V^{\prime}}{N_{b^{\prime}}}$.

Definição 2.31. Sejam $f: V \rightarrow V^{\prime}$ uma transformação linear e $\mathcal{B}, \mathcal{B}^{\prime}$ bases ordenadas de $V$ e $V^{\prime}$, respectivamente. Sendo $\mathcal{B}=\left\{v_{1}, \ldots, v_{n}\right\}$ então $M\left(f, \mathcal{B}^{\prime} \leftarrow \mathcal{B}\right)$ é a matriz cuja as colunas são as coordenadas de $f\left(v_{1}\right), \ldots, f\left(v_{n}\right)$ com respeito à $B^{\prime}$.

Em particular, se $f$ é a identidade de $V, \operatorname{Id}_{V}$, então teremos a matriz de transformação de $\mathcal{B}$ para $\mathcal{B}^{\prime}$. Vamos denotar $[b]_{\mathcal{B}}=M_{\mathcal{B}}(b)$.

Observação 2.32. 1. Sejam $\mathcal{B}$ e $\mathcal{B}^{\prime}$ bases de $V$ e $P=M\left(\operatorname{Id}_{V}, \mathcal{B} \leftarrow \mathcal{B}^{\prime}\right)$, então $M_{\mathcal{B}^{\prime}}(b)=P^{t} M_{\mathcal{B}}(b) P$ e, assim, $\operatorname{det}\left(M_{\mathcal{B}}(b)\right)=(\operatorname{det} P)^{2} \operatorname{det}\left(M_{\mathcal{B}}(b)\right)$. Em particular, o posto da matriz $M_{\mathcal{B}}(b)$ é independente de $\mathcal{B}$ e igual ao posto de b. Além disso, a forma bilinear simétrica $b$ é um produto escalar se, e só se, $\operatorname{det}\left(M_{\mathcal{B}}(b)\right) \neq 0($ para uma base $\mathcal{B}$, logo para todas) e, assim, todo produto escalar g é tal que

$$
(-1)^{\nu} \operatorname{det}\left(M_{\mathcal{B}}(g)\right)>0 \text {. }
$$

Demonstração. É fácil verificar o resultado considerando $\mathcal{B}$ uma base de Sylvester e a Observação 2.30, item 1..

$(\Rightarrow)$ Se $g$ é produto escalar então $N=\{0\}$ e a base $\mathcal{B}$ não possui vetores tipo-luz, logo $\operatorname{det}\left(M_{\mathcal{B}}(g)\right)= \pm 1$.

$(\Leftarrow)$ Se $\operatorname{det}\left(M_{\mathcal{B}}(g)\right) \neq 0$ e sabendo que $M_{\mathcal{B}}(g)=I_{\pi} \oplus-I_{\nu} \oplus 0_{\delta}$ então $\delta=0$, logo $N=\{0\}$ portanto $g$ é produto escalar.

2. Se g é produto escalar e $\left\{e_{1}, \ldots, e_{\pi}, e_{\pi+1}, \ldots, e_{\pi+\nu}\right\}$, com $\pi+\nu=n$ é uma base ortonormal qualquer, então:

$$
v=\sum_{i=1}^{n} \varepsilon_{i} g\left(e_{i}, v\right) e_{i}
$$

onde $\varepsilon_{i}=g\left(e_{i}, e_{i}\right)= \pm 1$. 
Demonstração. Vamos mostrar que $v-\sum_{i=1}^{n} \varepsilon_{i} g\left(e_{i}, v\right) e_{i}$ é ortogonal com toda a base, ou seja, pertence a $N$.

$$
\begin{aligned}
& g\left(v-\sum_{i=1}^{n} \varepsilon_{i} g\left(e_{i}, v\right) e_{i}, e_{j}\right)=g\left(v, e_{j}\right)-g\left(\sum_{i=1}^{n} \varepsilon_{i} g\left(e_{i}, v\right) e_{i}, e_{j}\right) \\
& =g\left(v, e_{j}\right)-\sum_{i=1}^{n} \varepsilon_{i} g\left(e_{i}, v\right) g\left(e_{i}, e_{j}\right)=g\left(v, e_{j}\right)-g\left(e_{j}, v\right)=0 .
\end{aligned}
$$

\subsection{Os isomorfismos flat e sharp}

Nessa seção usaremos a identificação entre o espaço e o seu bidual como vimos no Teorema 2.3 .

Definição 2.33. Seja $g$ um produto escalar em $V$, a aplicação flat $b: V \rightarrow V^{*}$ é definida por $b(v) \equiv v^{b}: V \rightarrow \mathbb{R}, v^{b}(w)=g(v, w)$, para qualquer $v, w \in V$.

$A$ aplicação sharp é definido por $\sharp: V^{*} \rightarrow V, \sharp(\omega) \equiv \omega^{\sharp}$, caracterizado por $g\left(\omega^{\sharp}, v\right)=\omega(v)$, para quaisquer $v \in V$ e $\omega \in V^{*}$.

Proposição 2.34. As aplicações b e $\sharp$ definidas acima são isomorfismos inversos.

Demonstração. Seja $V$ um espaço vetorial de dimensão finita $n$.

- $\operatorname{para} b: V \rightarrow V^{*}$,

1. está bem definida, sejam $v_{1}, v_{2} \in V$ tais que $v_{1}=v_{2}$, definimos

$$
b\left(v_{1}\right): V \rightarrow \mathbb{R}, w \longmapsto g\left(v_{1}, w\right) \quad \text { e } \quad b\left(v_{2}\right): V \rightarrow \mathbb{R}, w \longmapsto g\left(v_{2}, w\right) .
$$

Então

$$
b\left(v_{1}\right) w=g\left(v_{1}, w\right)=g\left(v_{2}, w\right)=b\left(v_{2}\right) w
$$

para todo $w \in V$.

2. é linear, sejam $v_{1}, v_{2} \in V$ e $\alpha \in \mathbb{R}$. Então

$$
b\left(v_{1}+\alpha v_{2}\right) w=g\left(v_{1}+\alpha v_{2}, w\right)=g\left(v_{1}, w\right)+\alpha g\left(v_{2}, w\right)=b\left(v_{1}\right) w+\alpha b\left(v_{2}\right) w .
$$

3. é injetora, suponha que $v \in V$ é tal que $b(v)=0$, logo para qualquer $w \in V$, $0=b(v) w=g(v, w)$, portanto $v=0$.

4. é sobrejetora, pois $\operatorname{dim} V<\infty$ e $\operatorname{dim} \operatorname{ker} b=0$.

- $\operatorname{para} \sharp: V^{*} \rightarrow V$,

1. está bem definida, sejam $\omega_{1}, \omega_{2} \in V^{*}$ tais que $\omega_{1}=\omega_{2}$. Então,

$$
g\left(\omega_{1}^{\sharp}, v\right)=\omega_{1}(v)=\omega_{2}(v)=g\left(\omega_{2}^{\sharp}, v\right),
$$

para qualquer $v \in V$. Logo, $\omega_{1}^{\sharp}=\omega_{2}^{\sharp}$. 
2. é linear, sejam $\omega_{1}, \omega_{2} \in V$ e $\alpha \in \mathbb{R}$. Então

$$
\begin{array}{r}
g\left(\left(\omega_{1}+\alpha \omega_{2}\right)^{\sharp}, v\right)=\left(\omega_{1}+\alpha \omega_{2}\right) v=\omega_{1}(v)+\alpha \omega_{2}(v)=g\left(\omega_{1}^{\sharp}, v\right)+g\left(\alpha \omega_{2}^{\sharp}, v\right) \\
=g\left(\omega_{1}^{\sharp}+\alpha \omega_{2}^{\sharp}, v\right)
\end{array}
$$

para qualquer $v \in V$.

3. é injetora, suponha $\omega \in V^{*}$ tal que $\omega^{\sharp}=0$, logo $0=g\left(\omega^{\sharp}, v\right)=\omega(v)$, para qualquer $v \in V$, portanto $\omega=0$.

4. é sobrejetora, pois $\operatorname{dim} V^{*}=\operatorname{dim} V<\infty$ e $\operatorname{dim} \operatorname{ker} \sharp=0$.

Portanto, b e \# são isomorfismos. Mostraremos agora que são inversos.

- temos $(b \circ \sharp)=\operatorname{Id}_{V^{*}}$, de fato

$$
(b \circ \sharp)(\omega)=b\left(\omega^{\sharp}\right): V \rightarrow \mathbb{R}, v \longmapsto g\left(\omega^{\sharp}, v\right)=\omega(v)
$$

$\log 0(b \circ \sharp) \omega=\omega$.

- temos $(\sharp \circ b)=\mathrm{Id}_{V}$, de fato

$$
(\sharp \circ b) v=\sharp\left(v^{b}\right),
$$

então

$$
g\left(\left(v^{b}\right)^{\sharp}, w\right)=v^{b}(w)=g(v, w),
$$

para todo $w \in V$. Logo $(\sharp \circ b) v=v$.

Observe que a transformação $b$ pode ser definida para qualquer forma bilinear, mesmo degenerada, mas assim $b$ não será injetora pois ker $b=N(b)$.

Esses isomorfismos podem ser escritos em coordenadas. Sejam $\mathcal{B}=\left\{e_{1}, \ldots, e_{n}\right\}$ uma base de $V$ e $\mathcal{B}^{*}=\left\{\varphi^{1}, \ldots, \varphi^{n}\right\}$ sua correspondente base dual. Denote

$$
g_{i j}=g\left(e_{i}, e_{j}\right) \quad \text { e } \quad g^{i j}=\left(M_{\mathcal{B}}(g)^{-1}\right)_{i j} .
$$

É válido lembrar que $M_{\mathcal{B}}(g)$ é inversível, pois $g$ é produto escalar e como mostrado na Observações 2.32 item $1 ., \operatorname{det}\left(M_{\mathcal{B}}(g)\right) \neq 0$.

Se $v=\sum_{i=1}^{n} a^{i} e_{i}$, então $v^{b} \in V^{*}$ e $v^{b}=\sum_{j=1}^{n} a_{j} \varphi^{j}$, onde

$$
a_{j}=v^{b}\left(e_{j}\right)=g\left(v, e_{j}\right)=\sum_{i=1}^{n} a^{i} g_{i j}
$$

Se $\omega=\sum_{j=1}^{n} b_{j} \varphi^{j}$, então $\sharp \omega \in V$ e $\omega^{\sharp}=\sum_{j=1}^{n} b^{j} v_{j}$, onde

$$
g\left(\omega^{\sharp}, v_{i}\right)=\omega\left(v_{i}\right)=b_{i} \quad \text { e } \quad g\left(\omega^{\sharp}, v_{i}\right)=g\left(\sum_{j=1}^{n} b^{j} v_{j}, v_{i}\right)=\sum_{j=1}^{n} b^{j} g_{j i} .
$$


De modo que temos o seguinte sistema

$$
\left[\begin{array}{c}
b_{1} \\
\vdots \\
b_{n}
\end{array}\right]=\left[\begin{array}{ccc}
g_{11} & \cdots & g_{n 1} \\
\vdots & \ddots & \vdots \\
g_{1 n} & \cdots & g_{n n}
\end{array}\right]\left[\begin{array}{c}
b^{1} \\
\vdots \\
b^{n}
\end{array}\right]
$$

sabemos ainda que $g_{i j}=g_{j i}$ para quaisquer $i, j$. Dessa forma teremos $b^{j}=\sum_{i=1}^{n} g^{i j} b_{i}$.

Observação 2.35. Seja $\left\{e_{1}, \ldots, e_{n-\nu}, e_{n-\nu+1}, \ldots, e_{n}\right\}$ uma base ortonormal, então

$$
g_{i j}=\delta_{i j} \epsilon_{i}, \quad \text { com } \quad \epsilon_{i}=\left\{\begin{array}{cc}
1 & \text { se } i<n-\nu+1 \\
-1 & \text { se } i \geq n-\nu+1
\end{array}\right.
$$

Logo $a^{i}=\varepsilon_{i} a_{i}$. Então, se g é um produto escalar euclidiano então $s_{i}=a_{i}$, para qualquer $i=1, \ldots, n$ e se g é um produto escalar lorentziano então $a^{i}=a_{i}$, para $i \leq n-1$ e $a^{n}=-a_{n}$.

\subsection{Derivada covariante}

Um dos conceitos principais desse trabalho é o de geodésica e para isso precisamos entender o que é derivada covariante, uma generalização de derivada direcional aplicada a vetores tangentes.

Definição 2.36. Seja $M$ uma variedade diferenciável. Definimos $\mathcal{F}(M)$ como sendo o conjunto das funções $f: M \rightarrow \mathbb{R}$.

Podemos definir em $\mathcal{F}(M)$ as operações de soma e multiplicação por escalar da seguinte forma.

1. Soma. $+: \mathcal{F}(M) \times \mathcal{F}(M) \rightarrow \mathcal{F}(M)$, dado por $(f, g) \mapsto f+g$, com $(f+g)(p)=f(p)+g(p)$, para todo $p \in M$.

2. Multiplicação por escalar. $: \mathbb{R} \times \mathcal{F}(M) \rightarrow \mathcal{F}(M)$, dado por $(\alpha, f) \mapsto \alpha \cdot f$, com $(\alpha \cdot f)(p)=\alpha f(p)$, para todo $p \in M$.

Proposição 2.37. O conjunto $\mathcal{F}(M)$, munido das operações de soma e multiplicação por escalar definidas acima, é um espaço vetorial.

Definição 2.38 (por derivações). Seja $p$ um ponto de uma variedade diferenciável $M$. $U m$ vetor tangente de $M$ em $p$ é um funcional linear $X_{p}: \mathcal{F}(M) \rightarrow \mathbb{R}$ tal que

i. é $\mathbb{R}$-linear: $X_{p}(\alpha f+\beta g)=\alpha X_{p}(f)+\beta X_{p}(g)$

ii. vale a Regra de Leibniz: $X_{p}(f g)=X_{p}(f) g(p)+f(p) X_{p}(g)$

para todos $\alpha, \beta \in \mathbb{R}$ e $f, g \in \mathcal{F}(M)$.

Definição 2.39. Sejam $M$ uma variedade diferenciável e $p \in M$. Definimos o espaço tangente de $M$ em $p$, denotado por $\mathrm{T}_{p}(M)$, como sendo o conjunto de todos os vetores tangentes a $M$ em $p$.

Definimos o ponto $0_{p}$ em $\mathrm{T}_{p} M$ como sendo o ponto de contato entre o espaço tangente e a variedade $M$. 
Podemos definir, em cada ponto $p \in M$ as seguintes operações:

1. soma, $\left(X_{p}+Y_{p}\right)(f)=X_{p}(f)+Y_{p}(f)$ e

2. multiplicação por escalar, $\left(\alpha X_{p}\right)(f)=\alpha\left(X_{p}(f)\right)$, para quaisquer $f \in \mathcal{F}(M)$ e $\alpha \in \mathbb{R}$. Assim, temos que $\mathrm{T}_{p} M$ é um espaço vetorial.

De modo equivalente, podemos definir vetores tangentes da seguinte forma:

Definição 2.40 (como derivadas direcionais). Seja $\gamma: I \rightarrow M$ uma curva diferenciável com $\gamma\left(t_{0}\right)=p$. $O$ vetor tangente à variedade diferenciável $M$ em $p$ é a função $\gamma^{\prime}\left(t_{0}\right)$ : $\mathcal{F}(M) \rightarrow \mathbb{R}$ definida por

$$
\gamma^{\prime}\left(t_{0}\right) f=(f \circ \gamma)^{\prime}\left(t_{0}\right)
$$

Definição 2.41. Seja $M$ uma variedade diferenciável. Podemos definir o fibrado tangente de $M$ como sendo o conjunto $\mathrm{T} M=\left\{\left(p, X_{p}\right) \mid p \in M\right.$ e $\left.X_{p} \in \mathrm{T}_{p} M\right\}$.

Como cada espaço tangente é um espaço vetorial, então é importante determinar a base de tal espaço.

Definição 2.42. Seja $\varphi=\left(x^{1}, \cdots, x^{n}\right)$ um sistema de coordenadas em $M$ ao redor de $p$, ou seja, $\varphi: M \rightarrow \mathbb{R}^{n}$, com $\varphi(p)=x_{0}$. Se $f \in \mathcal{F}(M)$, então

$$
\frac{\partial f}{\partial x^{i}}(p)=\frac{\partial\left(f \circ \varphi^{-1}\right)}{\partial x_{i}}\left(x_{0}\right)
$$

com $1 \geq i \geq n$, onde $x_{1}, \cdots, x_{n}$ são as coordenadas de $\mathbb{R}^{n}$.

Desse modo, é possível ver que a função

$$
\left.\partial_{i}\right|_{p}=\left.\frac{\partial}{\partial x^{i}}\right|_{p}: \mathcal{F}(M) \rightarrow \mathbb{R}
$$

definida por $f \in \mathcal{F}(M) \mapsto\left(\frac{\partial f}{\partial x^{i}}\right)(p)$, é um vetor tangente de $M$ em $p$ pela Definição 2.38.

Agora podemos mostrar que tais funções formam uma base para $\mathrm{T}_{p} M$.

Proposição 2.43. Sejam $M$ uma variedade diferenciável e $p$ um ponto em $M$. Então $\left\{\left.\partial_{1}\right|_{p}, \cdots,\left.\partial_{n}\right|_{p}\right\}$ é uma base para $\mathrm{T}_{p} M$.

Demonstração. Seja $(\varphi, U)$ uma vizinhança coordenada de $M$ ao redor de $p, \operatorname{como} \varphi(p)=x_{0}$. Considere $\gamma: I \rightarrow M$ uma curva diferenciável em $M$ com $\gamma\left(t_{0}\right)=p$ e $\gamma^{\prime}\left(t_{0}\right)=X_{p}$, onde $X_{p} \in \mathrm{T}_{p} M$. Seja $f \in \mathcal{F}(M)$, então

$$
\begin{aligned}
X_{p}(f)= & (f \circ \gamma)^{\prime}\left(t_{0}\right)=\left(f \circ \varphi^{-1} \circ \varphi \circ \gamma\right)^{\prime}\left(t_{0}\right)=d\left(f \circ \varphi^{-1}\right)_{\left(\varphi \circ \gamma\left(t_{0}\right)\right)}(\varphi \circ \gamma)^{\prime}\left(t_{0}\right) \\
& =d\left(f \circ \varphi^{-1}\right)_{x_{0}}(\varphi \circ \gamma)^{\prime}\left(t_{0}\right)=\sum_{i=1}^{n} \frac{\partial\left(f \circ \varphi^{-1}\right)}{\partial x_{i}}\left(x_{0}\right) \cdot \frac{d x^{i}}{d t}\left(t_{0}\right)=\left.\sum_{i=1}^{n} \frac{d x^{i}}{d t}\left(t_{0}\right) \cdot \partial_{i}\right|_{p}(f)
\end{aligned}
$$

Vamos mostrar agora que o conjunto $\left\{\left.\partial_{1}\right|_{p}, \cdots,\left.\partial_{n}\right|_{p}\right\}$ é linearmente independente. Suponha uma combinação tal que $\left.\sum \alpha^{i} \partial_{i}\right|_{p}=0$, com $\alpha_{i} \in \mathbb{R}$. Aplicando em cada função $x^{j}$ teremos que

$$
0=\sum_{i} \alpha^{i} \frac{\partial x^{j}}{\partial x^{i}}(p)=\sum_{i} \alpha^{i} \delta_{i j}=\alpha^{j} .
$$


Definição 2.44. Sejam $M$ uma variedade diferenciável e TM o fibrado tangente. Defina a projeção $\pi: \mathrm{T} M \rightarrow M$ como $X_{p} \in \mathrm{T}_{p} M \mapsto p \in M$, assim $\pi^{-1}(p)=\mathrm{T}_{p} M$.

Definido o que são vetores tangentes e os espaços tangentes podemos ampliar nosso estudo usando esses conceitos para caracterizar e estudar suas propriedades na variedade. Os campos vetoriais são ferramentas essencias no estudo das propriedades geométricas de uma variedade, pois descrevem características intrínsecas da variedade de modo intuitivo.

Definição 2.45. Um campo vetorial $X$ em uma variedade diferenciável $M$ é uma função que associa a cada ponto $p \in M$ um vetor tangente $X_{p}$ em $\mathrm{T}_{p} M$.

Se $X$ é um campo vetorial e $f \in \mathcal{F}(M)$, então $X f$ denota uma função em $M$ a valores reais dada por $(X f)(p)=X_{p}(f)$, para qualquer $p \in M$. Juntamente com a Definição 2.38, o campo vetorial pode ser interpretado como derivadas direcionais em cada ponto de $M$, então $X f$ é a derivada de $f$ na direção do campo $X$. O conjunto dos campos vetorias de uma variedade $M$ é denotado por $\mathfrak{X}(M)$.

Consequentemente, é natural pensar em derivada de ordem superior e considerar $X(Y f)$, entretanto não é sempre possível garantir que $X Y$ é um campo vetorial. Para isso precisamos definir o que é uma conexão de Levi-Civitta. Mas antes podemos definir uma operação mais simples que é sempre campo vetorial: o colchete de Lie.

Considere a seguinte operação com $X, Y \in \mathfrak{X}(M),[X, Y]=X Y-Y X$. Vamos mostrar que essa operação é um vetor tangente para cada $p \in M$ segundo a Definição 2.38. Sejam $\alpha, \beta \in \mathbb{R}$ e $f, g \in \mathcal{F}(M)$, então:

$$
\begin{gathered}
{[X, Y]_{p}(\alpha f+\beta g)=X_{p}(Y(\alpha f+\beta g))-Y_{p}(X(\alpha f+\beta g))} \\
=\alpha X_{p}(Y f)+\beta X_{p}(Y g)-\alpha Y_{p}(X f)-\beta Y_{p}(X g) \\
=\alpha\left(X_{p}(Y f)-Y_{p}(X f)\right)+\beta\left(X_{p}(Y g)-Y_{p}(X g)\right) \\
=\alpha[X, Y]_{p}(f)+\beta[X, Y]_{p}(g) . \\
{[X, Y]_{p}(f g)=X_{p}(Y(f g))-Y_{p}(X(f g))} \\
=X_{p}(Y f \cdot g(p)+f(p) \cdot Y g)-Y_{p}(X f \cdot g(p)+f(p) \cdot X g) \\
=X_{p}(Y f) \cdot g(p)+f(p) \cdot X_{p}(Y g)-Y_{p}(X f) \cdot g(p)-f(p) \cdot Y_{p}(X g) \\
=[X, Y]_{p}(f) \cdot g(p)+f(p) \cdot[X, Y]_{p}(g) .
\end{gathered}
$$

Dessa forma, fica provado que $[X, Y] \in \mathfrak{X}(M)$ para $X, Y \in \mathfrak{X}(M)$.

Definição 2.46. Sejam $X, Y \in \mathfrak{X}(M)$. O colchete de Lie de $X$ e $Y$, denotado por $[X, Y]$, é um campo vetorial que associa a cada $p \in M$ o vetor tangente $[X, Y]_{p}$ tal que, para qualquer $f \in \mathcal{F}(M)$

$$
[X, Y]_{p}(f)=X_{p}(Y f)-Y_{p}(X f) .
$$

De agora em diante omitiremos o ponto onde consideramos o campo vetorial a menos que seja necessário.

Proposição 2.47. Sejam $X, Y, Z \in \mathfrak{X}(M), \alpha, \beta \in \mathbb{R}$. Então:

i. $[X, Y]=-[Y, X]$

ii. $[\alpha X+\beta Y, Z]=\alpha[X, Z]+\beta[Y, Z] e[X, \alpha Y+\beta Z]=\alpha[X, Y]+\beta[X, Z]$; 
iii. $[[X, Y], Z]+[[Y, Z], X]+[[Z, X], Y]=0$.

Demonstração. $\quad$ i. $[X, Y]+[Y, X]=X Y-Y X+Y X-X Y=0, \operatorname{logo}[X, Y]=-[Y, X]$; ii.

$$
\begin{array}{r}
{[\alpha X+\beta Y, Z]=(\alpha X+\beta Y) Z-Z(\alpha X+\beta Y)=(\alpha X) Z+(\beta Y) Z-Z(\alpha X)-Z(\beta Y)} \\
=\alpha(X Z)+\beta(Y Z)-\alpha(Z X)-\beta(Z Y)=\alpha(X Z-Z X)+\beta(Y Z-Z Y) \\
=\alpha([X, Z])+\beta([Y, Z])
\end{array}
$$

iii.

$$
\begin{aligned}
& {[[X, Y], Z]=[X Y-Y X, Z]=[X Y, Z]-[Y X, Z]=X Y Z-Z X Y-Y X Z+Z Y X} \\
& {[[Y, Z], X]=[Y Z-Z Y, X]=[Y Z, X]-[Z Y, X]=Y Z X-X Y Z-Z Y X+X Z Y} \\
& {[[Z, X], Y]=[Z X-X Z, Y]=[Z X, Y]-[X Z, Y]=Z X Y-Y Z X-X Z Y+Y X Z}
\end{aligned}
$$

Então

$$
\begin{aligned}
{[[X, Y], Z]+[[Y, Z], X]+[[Z, X], Y]=} & X Y Z-Z X Y-Y X Z+Z Y X \\
& +Y Z X-X Y Z-Z Y X+X Z Y \\
& +Z X Y-Y Z X-X Z Y+Y X Z \\
= & 0 .
\end{aligned}
$$

Proposição 2.48. Sejam $X, Y \in \mathfrak{X}(M)$ e $f, g \in \mathcal{F}(M)$, então

$$
[f X, g Y]=f g[X, Y]+f(X g) Y-g(Y f) X .
$$

Demonstração. Sejam $h \in \mathcal{F}(M)$ e $p \in M$, quaisquer, então

$$
\begin{array}{r}
{[f X, g Y]_{p}(h)=(f X)_{p}(g \cdot(Y h)(p))-(g Y)_{p}(f \cdot(X h)(p))} \\
=f(p) X_{p}(g \cdot(Y h)(p))-g(p) Y_{p}(f \cdot(X h)(p)) \\
=f(p)\left\{X_{p}(g) \cdot(Y h)(p)+g(p) \cdot X_{p}(Y h)(p)\right\}-g(p)\left\{Y_{p}(f) \cdot(X h)(p)+f(p) \cdot Y_{p}(X h)(p)\right\} \\
=f(p) g(p)\left\{X_{p}(Y h)(p)-Y_{p}(X h)(p)\right\}+f(p) X_{p}(g) \cdot(Y h)(p)-g(p) Y_{p}(f) \cdot(X h)(p) \\
=f(p) g(p)[X, Y](h)(p)+f(p) X_{p}(g) \cdot(Y h)(p)-g(p) Y_{p}(f) \cdot(X h)(p) .
\end{array}
$$

Podemos agora definir o que é uma conexão, que traz a noção de derivada superior para campos vetorias. 
Definição 2.49. Seja $M$ uma variedade diferenciável. Uma conexão $\nabla$ em $M$ é uma função

$$
\nabla: \mathfrak{X}(M) \times \mathfrak{X}(M) \rightarrow \mathfrak{X}(M),
$$

definida por $(X, Y) \mapsto \nabla_{X} Y$, tal que valem:

i. $\nabla$ é $\mathcal{F}(M)$-linear na primeira coordenada, ou seja,

$$
\nabla_{f X+g Y} Z=f \nabla_{X} Z+g \nabla_{Y} Z
$$

ii. $\nabla$ é $\mathbb{R}$-linear na segunda coordenada, ou seja,

$$
\nabla_{X}(Y+Z)=\nabla_{X} Y+\nabla_{X} Z
$$

iii. Se a regra de Leibniz:

$$
\nabla_{X}(f Y)=f \nabla_{X} Y+(X f) Y
$$

para $X, Y, Z \in \mathfrak{X}(M)$ e $f, g \in \mathcal{F}(M)$.

Dizemos que $\nabla_{X} Y$ é a derivada covariante do campo $Y$ na direção do campo $X$.

Tomando as definições e resultados da Seção 2.2 podemos definir algo semelhante ao que foi feito na Definição 2.45 só que para tensores.

Definição 2.50. Um $(r, s)$-campo tensorial $A$ em uma variedade diferenciável $M$ é uma função que associa a cada ponto $p \in M$ um $(r, s)$-tensor $A_{p}$ em $\mathrm{T}_{p} M$.

$O$ conjunto dos $(r, s)$-campos tensoriais de $M$ será denotado por $\mathfrak{T}_{s}^{r}(M)$.

Pela Definição 2.15 sabemos que um produto escalar é um 2-tensor covariante definido em um espaço vetorial $V$.

Definição 2.51. Seja $M$ uma variedade diferenciável. Uma métrica de $M$ é um 2-campo tensorial covariante. Ou seja, se g é uma métrica de $M$ então $g \in \mathfrak{T}_{2}(M)$ em que a cada ponto $p \in M$ associa um produto escalar $g_{p}$ em $\mathrm{T}_{p} M$ tal que o indice de todo produto escalar é igual em todo $p$.

Note que todos as definições e resultados obtidos para produtos escalares podem ser estendidos para a métrica, tomando apenas o cuidado de lembrar que são resultados pontuais. Dessa forma, iremos omitir os índices $p$ quando não houver necessidade de citá-los. Além disso, usaremos, convenientemente, a notação $\langle\cdot, \cdot\rangle$ para métricas.

A métrica da variedade não influenciou nas definições e nos resultados dessa seção, entretanto o próximo Teorema mostra a existência de uma única conexão que se relaciona com a métrica da variedade, tal conexão é dita ser de Levi-Civita. Embora estamos considerando variedades e métricas semi-Riemannianas nesse trabalho, o teorema seguinte e conceitos anteriores são igualmente válidos para o caso Riemanniano.

Teorema 2.52. Seja $(M,\langle\cdot, \cdot\rangle)$ uma variedade semi-Riemanniana. Então existe uma única conexão $\nabla$ tal que:

iv. $\nabla$ é simétrica, ou seja,

$$
[X, Y]=\nabla_{X} Y-\nabla_{Y} X
$$

v. $\nabla$ paraleliza $\langle\cdot, \cdot\rangle$, ou seja,

$$
X\langle Y, Z\rangle=\left\langle\nabla_{X} Y, Z\right\rangle+\left\langle Y, \nabla_{X} Z\right\rangle
$$


para $X, Y, Z \in \mathfrak{X}(M)$. $\nabla$ é chamado de conexão de Levi-Civita de $M$ e é caracterizado pela seguinte expressão, chamada fórmula de Koszul,

$$
2\left\langle\nabla_{X} Y, Z\right\rangle=X\langle Y, Z\rangle+Y\langle Z, X\rangle-Z\langle X, Y\rangle-\langle X,[Y, Z]\rangle+\langle Y,[Z, X]\rangle+\langle Z,[X, Y]\rangle .
$$

Demonstração. Nós iremos mostrar que dada uma conexão, a validade da fórmula de Koszul acontece se, e somente se, valem os itens $i v$. e $v$. Além disso, mostraremos que tal conexão é única. Começaremos mostrando a unicidade.

Suponha que $\nabla$ é uma conexão de Levi-Civita, logo valem os itens $i v$. e $v$. acima. Pelo lado direito da fórmula de Koszul temos então

$$
\begin{aligned}
& X\langle Y, Z\rangle+ Y\langle Z, X\rangle-Z\langle X, Y\rangle-\langle X,[Y, Z]\rangle+\langle Y,[Z, X]\rangle+\langle Z,[X, Y]\rangle \\
&=\left\langle\nabla_{X} Y, Z\right\rangle+\left\langle Y, \nabla_{X} Z\right\rangle+\left\langle\nabla_{Y} Z, X\right\rangle+\left\langle Z, \nabla_{Y} X\right\rangle-\left\langle\nabla_{Z} X, Y\right\rangle-\left\langle X, \nabla_{Z} Y\right\rangle \\
&-\left\langle X, \nabla_{Y} Z-\nabla_{Z} Y\right\rangle+\left\langle Y, \nabla_{Z} X-\nabla_{X} Z\right\rangle+\left\langle Z, \nabla_{X} Y-\nabla_{Y} X\right\rangle \\
&=\left\langle\nabla_{X} Y, Z\right\rangle+\left\langle Y, \nabla_{X} Z\right\rangle+\left\langle\nabla_{Y} Z, X\right\rangle+\left\langle Z, \nabla_{Y} X\right\rangle-\left\langle\nabla_{Z} X, Y\right\rangle-\left\langle X, \nabla_{Z} Y\right\rangle \\
& \quad-\left\langle X, \nabla_{Y} Z\right\rangle+\left\langle X, \nabla_{Z} Y\right\rangle+\left\langle Y, \nabla_{Z} X\right\rangle-\left\langle Y, \nabla_{X} Z\right\rangle+\left\langle Z, \nabla_{X} Y\right\rangle-\left\langle Z, \nabla_{Y} X\right\rangle
\end{aligned}
$$

Logo $\nabla$ satifaz a fórmula de Koszul e ainda, note que $\left\langle\nabla_{X} Y, Z\right\rangle=\left(\nabla_{X} Y\right)^{b} Z$. E como b é um isomorfismo, $\log 0 \nabla_{X} Y$ é único.

Para provar a existência, consideremos a função $F: \mathfrak{X}(M) \times \mathfrak{X}(M) \times \mathfrak{X}(M) \rightarrow \mathbb{R}$, definida por

$$
F(X, Y, Z)=X\langle Y, Z\rangle+Y\langle Z, X\rangle-Z\langle X, Y\rangle-\langle X,[Y, Z]\rangle+\langle Y,[Z, X]\rangle+\langle Z,[X, Y]\rangle
$$

Fixado os campos $X, Y \in \mathfrak{X}(M)$, temos que a aplicação $Z \mapsto F(X, Y, Z)$ é $\mathcal{F}(M)$-linear. De fato, para $Z, W \in \mathfrak{X}(M)$ e $f, g \in \mathcal{F}(M)$,

$$
\begin{gathered}
F(X, Y, f Z+g W)=X\langle Y, f Z+g W\rangle+Y\langle f Z+g W, X\rangle-(f Z+g W)\langle X, Y\rangle \\
-\langle X,[Y, f Z+g W]\rangle+\langle Y,[f Z+g W, X]\rangle+\langle f Z+g W,[X, Y]\rangle \\
=f X\langle Y, Z\rangle+g X\langle Y, W\rangle+f Y\langle Z, X\rangle+g Y\langle W, X\rangle-f Z\langle X, Y\rangle-g W\langle X, Y\rangle \\
-\langle X,[Y, f Z]+[Y, g W]\rangle+\langle Y,[f Z, X]+[g W, X]\rangle+\langle f Z+g W,[X, Y]\rangle \\
=f\{X\langle Y, Z\rangle+Y\langle Z, X\rangle-Z\langle X, Y\rangle-\langle X,[Y, Z]\rangle+\langle Y,[Z, X]\rangle+\langle Z,[X, Y]\rangle\} \\
+g\{X\langle Y, W\rangle+Y\langle W, X\rangle-W\langle X, Y\rangle-\langle X,[Y, W]\rangle+\langle Y,[W, X]\rangle+\langle W,[X, Y]\rangle\} \\
=f F(X, Y, Z)+g F(X, Y, W) .
\end{gathered}
$$

Dessa forma, $F$ é uma 1-forma, então existe um único campo, denotado por $\nabla_{X} Y$, tal que $\left(\nabla_{X} Y\right)^{b} Z=F(X, Y, Z)$ e $F(X, Y, Z)=2\left\langle\nabla_{X} Y, Z\right\rangle$. Vamos mostrar que $\nabla$ é conexão de Levi-Civita. 
i. $\nabla$ é $\mathcal{F}(M)$-linear na primeira coordenada,

$$
\begin{gathered}
2\left\langle\nabla_{f X+g Y} Z, W\right\rangle=F(f X+g Y, Z, W)=(f X+g Y)\langle Z, W\rangle+Z\langle W, f X+g Y\rangle \\
-W\langle f X+g Y, Z\rangle-\langle f X+g Y,[Z, W]\rangle+\langle Z,[W, f X+g Y]\rangle+\langle W,[f X+g Y, Z]\rangle \\
=f(X\langle Z, W\rangle+Z\langle W, X\rangle-W\langle X, Z\rangle-\langle X,[Z, W]\rangle)+g(Y\langle Z, W\rangle+Z\langle W, Y\rangle \\
-W\langle Y, Z\rangle-\langle Y,[Z, W]\rangle)+\langle Z, W(f X)\rangle-\langle Z,(f X) W\rangle+\langle Z, W(g Y)\rangle-\langle Z,(g Y) W\rangle \\
+\langle W,(f X) Z\rangle-\langle W, Z(f X)\rangle+\langle W,(g Y) Z\rangle-\langle W, Z(g Y)\rangle \\
=f(X\langle Z, W\rangle+Z\langle W, X\rangle-W\langle X, Z\rangle-\langle X,[Z, W]\rangle+\langle Z,[W, X]\rangle+\langle W,[X, Z]\rangle) \\
+g(Y\langle Z, W\rangle+Z\langle W, Y\rangle-W\langle Y, Z\rangle-\langle Y,[Z, W]\rangle+\langle Z,[W, Y]\rangle+\langle W,[Y, Z]\rangle) \\
=f F(X, Z, W)+g F(Y, Z, W)=2 f\left\langle\nabla_{X}, Y, W\right\rangle+2 g\left\langle\nabla_{Y} Z, W\right\rangle=2\left\langle f \nabla_{X} Y+g \nabla_{Y} Z, W\right\rangle .
\end{gathered}
$$

ii. $\nabla$ é $\mathbb{R}$-linear na segunda coordenada,

$$
\begin{array}{r}
2\left\langle\nabla_{X}(Y+Z), W\right\rangle=F(X, Y+Z, W)=X\langle Y+Z, W\rangle+(Y+Z)\langle W, X\rangle-W\langle X, Y+Z\rangle \\
-\langle X,[Y+Z, W]\rangle+\langle Y+Z,[W, X]\rangle+\langle W,[X, Y+Z]\rangle \\
=X\langle Y, W\rangle+X\langle Z, W\rangle+Y\langle W, X\rangle+Z\langle W, X\rangle-W\langle X, Y\rangle-W\langle X, Z\rangle \\
-\langle X,[Y, W]\rangle-\langle X,[Z, W]\rangle+\langle Y,[W, X]\rangle+\langle Z,[W, X]\rangle+\langle W,[X, Y]\rangle+\langle W,[X, Z]\rangle \\
=F(X, Y, W)+F(X, Z, W)=2\left\langle\nabla_{X} Y+\nabla_{X} Z, W\right\rangle .
\end{array}
$$

iii.

$$
\begin{array}{r}
2\left\langle\nabla_{X}(f Y), Z\right\rangle=2 F(X, f Y, Z)=X\langle f Y, Z\rangle+f Y\langle Z, X\rangle-Z\langle X, f Y\rangle \\
-\langle X,[f Y, Z]\rangle+\langle f Y,[Z, X]\rangle+\langle Z,[X, f Y]\rangle \\
=X\langle f Y, Z\rangle+f Y\langle Z, X\rangle-Z\langle X, f Y\rangle-\langle X, f[Y, Z]-(Z f) Y\rangle \\
+\langle f Y,[Z, X]\rangle+\langle Z, f[X, Y]+(X f) Y\rangle \\
=f X\langle Y, Z\rangle+X f\langle Y, Z\rangle+f Y\langle Z, X\rangle-f Z\langle X, Y\rangle-Z f\langle X, Y\rangle-f\langle X,[Y, Z]\rangle \\
+\langle X,(Z f) Y\rangle+f\langle Y,[Z, X]\rangle+f\langle Z,[X, Y]\rangle+\langle Z,(X f) Y\rangle \\
=f(X\langle Y, Z\rangle+Y\langle Z, X\rangle-Z\langle X, Y\rangle-\langle X,[Y, Z]\rangle+\langle Y,[Z, X]\rangle+\langle Z,[X, Y]\rangle) \\
+2\langle Z,(X f) Y\rangle \\
=f F(X, Y, Z)+2\langle(X f) Y, Z\rangle=2 f\left\langle\nabla_{X} Y, Z\right\rangle+2\langle(X f) Y, Z\rangle
\end{array}
$$

$i v . \nabla$ é simétrica,

$$
\begin{gathered}
2\left\langle\nabla_{X} Y-\nabla_{Y} X, Z\right\rangle=F(X, Y, Z)-F(Y, X, Z) \\
=X\langle Y, Z\rangle+Y\langle Z, X\rangle-Z\langle X, Y\rangle-\langle X,[Y, Z]\rangle+\langle Y,[Z, X]\rangle+\langle Z,[X, Y]\rangle \\
-Y\langle X, Z\rangle-X\langle Z, Y\rangle+Z\langle Y, X\rangle+\langle Y,[X, Z]\rangle-\langle X,[Z, Y]\rangle-\langle Z,[Y, X]\rangle \\
=X\langle Y, Z\rangle-X\langle Y, Z\rangle+Y\langle Z, X\rangle-Y\langle Z, X\rangle-Z\langle X, Y\rangle+Z\langle X, Y\rangle \\
-\langle X,[Y, Z]\rangle+\langle X,[Y, Z]\rangle+\langle Y,[Z, X]\rangle-\langle Y,[X, Z]\rangle+\langle Z,[X, Y]\rangle+\langle Z,[X, Y]\rangle \\
=2\langle[X, Y], Z\rangle
\end{gathered}
$$


v. $\nabla$ paraleliza $g$

$$
\begin{gathered}
2\left\langle\nabla_{X} Y, Z\right\rangle+2\left\langle\nabla_{X} Z, Y\right\rangle=F(X, Y, Z)+F(X, Z, Y) \\
=X\langle Y, Z\rangle+Y\langle Z, X\rangle-Z\langle X, Y\rangle-\langle X,[Y, Z]\rangle+\langle Y,[Z, X]\rangle+\langle Z,[X, Y]\rangle \\
+X\langle Z, Y\rangle+Z\langle Y, X\rangle-Y\langle X, Z\rangle-\langle X,[Z, Y]\rangle+\langle Z,[Y, X]\rangle+\langle Y,[X, Z]\rangle \\
=2 X\langle Y, Z\rangle
\end{gathered}
$$

Portanto, fica provado a existência e unicidade de uma conexão de Levi-Civita associada à uma métrica $g$.

Exemplo 2.53. O exemplo mais simples de uma conexão de Levi-Civita é definida na variedade $\mathbb{R}^{n}$ com a métrica do Exemplo 2.25

$$
g(X, Y)=\left(\sum_{i=1}^{n-1} X^{i} Y^{j}\right)-X^{n} Y^{n}
$$

Sendo $X$ e $Y$ campos vetoriais em $\mathbb{R}^{n}$, definimos a função $\nabla: \mathfrak{X}\left(\mathbb{R}^{n}\right) \times \mathfrak{X}\left(\mathbb{R}^{n}\right) \rightarrow \mathfrak{X}\left(\mathbb{R}^{n}\right)$ por

$$
\nabla_{X} Y=\sum X\left(Y^{i}\right) \partial_{i}
$$

Vamos mostrar que $\nabla$ é, de fato, uma conexão de Levi-Civita.

i. sejam $X, Y, Z \in \mathfrak{X}\left(\mathbb{R}^{n}\right)$ e $f, g \in \mathcal{F}\left(\mathbb{R}^{n}\right)$,

$$
\nabla_{f X+g Y} Z=\sum(f X+g Y)\left(Z^{i}\right) \partial_{i}=\sum f X\left(Z^{i}\right) \partial_{i}+\sum g Y\left(W^{i}\right) \partial_{i}=f \nabla_{X} W+g \nabla_{Y} W
$$

ii. sejam $X, Y, Z \in \mathfrak{X}\left(\mathbb{R}^{n}\right)$,

$$
\nabla_{X}(Y+Z)=\sum X\left(Y^{i}+W^{i}\right) \partial_{i}=\sum X\left(Y^{i}\right) \partial_{i}+\sum X\left(W^{i}\right) \partial_{i}=\nabla_{X} Y+\nabla_{X} W
$$

iii. sejam $X, Y \in \mathfrak{X}\left(\mathbb{R}^{n}\right)$ e $f \in \mathcal{F}\left(\mathbb{R}^{n}\right)$,

$$
\nabla_{X} f Y=\sum X\left(f Y^{i}\right) \partial_{i}=\sum(X f) Y^{i} \partial_{i}+\sum(f X)\left(Y^{i}\right) \partial_{i}=(X f) Y+f \nabla_{X} Y .
$$

iv. $\operatorname{sejam} X, Y \in \mathfrak{X}\left(\mathbb{R}^{n}\right)$,

$$
\nabla_{X} Y-\nabla_{Y} X=\sum X\left(Y^{i}\right) \partial_{i}-\sum Y\left(X^{i}\right) \partial_{i}=X\left[\sum Y^{i} \partial_{i}\right]-Y\left[\sum X^{i} \partial_{i}\right]=X Y-Y X
$$

v. sejam $X, Y, Z \in \mathfrak{X}\left(\mathbb{R}^{n}\right)$,

$$
\begin{aligned}
X g(Y, Z)=X\left[\left(\sum_{i=1}^{n-1} Y^{i} Z^{j}\right)-Y^{n} Z^{n}\right]=\sum X\left(Y^{i} Z^{j}\right)-X\left(Y^{n} Z^{n}\right) \\
=\sum X\left(Y^{i}\right) Z^{j}+Y^{i} X\left(Z^{j}\right)-\left[X\left(Y^{n}\right) Z^{n}+Y^{n} X\left(Z^{j}\right)\right] \\
=\left[\sum X\left(Y^{i}\right) Z^{j}-X\left(Y^{n}\right) Z^{n}\right]-\left[\sum Y\left(X^{i}\right) Z^{j}-Y\left(X^{n}\right) Z^{n}\right] \\
=g\left(\nabla_{X} Y, Z\right)-g\left(Y, \nabla_{X} Z\right) .
\end{aligned}
$$


Portanto, pela Definição 2.49 e pelo Teorema 2.52, $\nabla$ definida em 2.1 é uma conexão de Levi-Civita.

Definição 2.54. Seja $(M,\langle\cdot, \cdot\rangle)$ uma variedade munida de um produto escalar, com $\operatorname{dim} M=n$. Seja $\nabla$ uma conexão de Levi-Civita associada à $\langle\cdot, \cdot\rangle$. Definimos os símbolos de Chirstoffel como sendo as funções $\Gamma_{i j}^{k}: M \rightarrow \mathbb{R}$, com $1 \leq i, j, k \leq n$, tais que

$$
\nabla_{\partial_{i}} \partial_{j}=\sum_{k=1}^{n} \Gamma_{i j}^{k} \partial_{k}
$$

onde $\left\{\partial_{1}, \ldots, \partial_{n}\right\}$ é a base canônica de $\mathrm{T}_{p} M$.

Usando a fórmula de Koszul podemos calcular os símbolos de Christoffel, $\Gamma_{i j}^{k} \in \mathcal{F}(M)$,

$$
\begin{array}{r}
2\left\langle\nabla_{\partial_{i}} \partial_{j}, \partial_{l}\right\rangle=\partial_{i}\left\langle\partial_{j}, \partial_{l}\right\rangle+\partial_{j}\left\langle\partial_{l}, \partial_{i}\right\rangle-\partial_{l}\left\langle\partial_{i}, \partial_{j}\right\rangle-\left\langle\partial_{i},\left[\partial_{j}, \partial_{l}\right]\right\rangle+\left\langle\partial_{j},\left[\partial_{l}, \partial_{i}\right]\right\rangle+\left\langle\partial_{l},\left[\partial_{i}, \partial_{j}\right]\right\rangle \\
=\partial_{i} g_{j l}+\partial_{j} g_{i l}-\partial_{l} g_{i j}
\end{array}
$$

Por outro lado, temos que

$$
2\left\langle\nabla_{\partial_{i}} \partial_{j}, \partial_{l}\right\rangle=2\left\langle\sum_{k=1}^{n} \Gamma_{i j}^{k} \partial_{k}, \partial_{l}\right\rangle=2 \sum_{k=1}^{n} \Gamma_{i j}^{k} g_{k l} .
$$

Dessa forma, com

$$
2 \sum_{k=1}^{n} \Gamma_{i j}^{k} g_{k l}=\partial_{i} g_{j l}+\partial_{j} g_{i l}-\partial_{l} g_{i j}
$$

obtemos que

$$
\Gamma_{i j}^{k}=\frac{1}{2} \sum_{l=1}^{n} g^{k l}\left(\partial_{i} g_{j l}+\partial_{j} g_{i l}-\partial_{l} g_{i j}\right) .
$$

Do item $i v$. do Teorema 2.52 temos que

$$
0=\left[\partial_{i}, \partial_{j}\right]=\nabla_{\partial_{i}} \partial_{j}-\nabla_{\partial_{j}} \partial_{i},
$$

então $\nabla_{\partial_{i}} \partial_{j}=\nabla_{\partial_{j}} \partial_{i}$. Portanto, $\Gamma_{i j}^{k}=\Gamma_{j i}^{k}$.

Exemplo 2.55. Seja a conexão $\nabla$ definida no Exemplo 2.53, dada por 2.1, em $\mathbb{R}^{n}$. É fácil ver que os símbolos de Christoffel para $\nabla$ são todos iguais a zero.

De fato, como $g(x, y)=\sum x^{i} y^{j}-x^{n} y^{n}$, para quaisquer $x, y \in \mathbb{R}^{n}$, então

$$
g_{i j}=g^{i j}=\left[\begin{array}{cccc}
1 & \cdots & 0 & 0 \\
\vdots & \ddots & \vdots & \vdots \\
0 & \cdots & 1 & 0 \\
0 & \cdots & 0 & -1
\end{array}\right]
$$

Pela equação 2.3 teremos que

$$
\Gamma_{i j}^{k}=\frac{1}{2} g^{k k}\left(\partial_{i} g_{j k}+\partial_{j} g_{i k}-\partial_{k} g_{i j}\right)=0,
$$

para todos $1 \leq i, j, k \leq n$. 
Usando as equações 2.2 e 2.3 podemos escrever uma forma genérica de uma conexão $\nabla$. Sejam $X, Y \in \mathfrak{X}(M)$ e $\left\{\partial_{1}, \cdots, \partial_{n}\right\}$ a base canônica de $\mathrm{T}_{p} M, \operatorname{logo}$

$$
X=\sum_{i=1}^{n} X^{i} \partial_{i} \quad \text { e } \quad Y=\sum_{j=1}^{n} Y^{j} \partial_{j}
$$

Então

$$
\begin{array}{r}
\nabla_{X} Y=\nabla_{\left(\sum_{i} X^{i} \partial_{i}\right)}\left(\sum_{j} Y^{j} \partial_{j}\right)=\sum_{i} X^{i} \nabla_{\partial_{i}}\left(\sum_{j} Y^{j} \partial_{j}\right)=\sum_{i, j} X^{i} \nabla_{\partial_{i}}\left(Y^{j} \partial_{j}\right) \\
=\sum_{i, j} X^{i}\left(\partial_{i}\left(Y^{j}\right) \partial_{j}+Y^{j} \nabla_{\partial_{i}} \partial_{j}\right)= \\
\sum_{i, j} X^{i}\left(\partial_{i}\left(Y^{j}\right) \partial_{j}+Y^{j} \sum_{k} \Gamma_{i j}^{k} \partial_{k}\right) \\
=\sum_{i, k} X^{i}\left[\partial_{i}\left(Y^{k}\right)+\sum_{j} Y^{j} \Gamma_{i j}^{k}\right] \partial_{k}
\end{array}
$$

\subsection{Derivada covariante ao longo de uma curva}

Um campo vetorial ao longo de uma curva é a restrição de um campo por uma curva na variedade. Dessa forma. considere $X$ um campo vetorial em $M$ escrito como $X=\sum_{i=1}^{n} X^{i} \partial_{i}$, sendo $X^{i} \in \mathcal{F}(M)$ e seja curva $\gamma: I \rightarrow M$, então a restrição de $X$ a curva $\gamma$ é o campo $\left.X\right|_{\gamma}: I \rightarrow M$ escrito como

$$
X(\gamma(t))=\left.\sum_{i=1}^{n} X^{i}(\gamma(t)) \partial_{i}\right|_{\gamma(t)}
$$

É conveniente em muitos momentos eliminar o $\gamma$ da notação desde que fique claro a curva, escrevendo somente $X(t)$.

Vimos na Definição 2.40 como definir vetores tangentes usando derivadas direcionais em curvas, de fato o conjunto dos vetores tangentes à uma curva formam um campo vetorial ao longo da curva. Desse modo, seja $\gamma$ uma curva em $M$, podemos definir o campo vetorial $\gamma^{\prime}$ ao longo de $\gamma$, escrito da seguinte forma

$$
\gamma^{\prime}(t)=\sum_{i=1}^{n} \frac{d \gamma^{i}}{d t}(t) \partial_{i}(t)
$$

onde denotamos $\gamma^{i}=x^{i} \circ \gamma$, para um sistema de coordenadas $\varphi=\left(x^{1}, \cdots, x^{n}\right)$. O campo $\gamma^{\prime}$ é comumente chamado de campo tangente.

Dessa forma, podemos definir a derivada covariante sob curvas, que nada mais é do que a derivada covariante de campos vista anteriormente, mas se tratando de um campo vetorial ao longo de uma curva fixada.

Definição 2.56. Sejam $\gamma$ uma curva em $M$ e $X$ um campo vetorial tal que $Y=X \circ \gamma$, a derivada covariante ao longo de $\gamma$, denotada por $\frac{D}{d t}$, é definida por

$$
\frac{D}{d t} \cdot=\nabla_{\gamma^{\prime}(t)} . \quad \text { e assim, } \quad \frac{D}{d t} Y=\nabla_{\gamma^{\prime}(t)} X
$$


Em coordenadas locais,

$$
\frac{D}{d t} Y(t)=\frac{D}{d t}\left\{\sum_{i=1}^{n} X^{i}(t) \partial_{i}(t)\right\}=\sum_{i=1}^{n}\left\{\frac{d}{d t} Y^{i}(t) \partial_{i}(t)+Y^{i}(t) \frac{D}{d t} \partial_{i}(t)\right\}
$$

precisamos agora saber como escrever $\frac{D}{d t} \partial_{i}(t)$. Logo,

$$
\left.\frac{D}{d t} \partial_{j}(t)=\nabla_{\gamma^{\prime}(t)} \partial_{j}(t)=\nabla \sum_{i=1}^{n} \frac{d \gamma^{i}}{d t}(t) \partial_{i}(t)\right\} \partial^{\partial_{j}(t)=\sum_{i=1}^{n}\left\{\frac{d \gamma^{i}}{d t}(t) \nabla_{\partial_{i}(t)} \partial_{j}(t)\right\}}
$$

agora, usando 2.2 temos

$$
\frac{D}{d t} Y(t)=\sum_{k=1}^{n}\left\{\frac{d}{d t} Y^{k}(t)+\sum_{i, j=1}^{n} Y^{j}(t) \frac{d}{d t} \gamma^{i}(t) \Gamma_{i j}^{k}(t)\right\} \partial_{k}(t)
$$

Note que podemos concluir a equação 2.7 usando 2.4 e tomando $X=\gamma^{\prime}$, nesse caso teremos

$$
\frac{D}{d t} Y=\sum_{i, k}\left[\partial_{i}\left(Y^{k}\right) \frac{d \gamma^{i}}{d t}+\sum_{j} \frac{d \gamma^{i}}{d t} Y^{j} \Gamma_{i j}^{k}\right] \partial_{k}
$$

porém, sendo $\psi:\left(x_{1}, \ldots, x_{n}\right) \mapsto \psi\left(x_{1}, \ldots, x_{n}\right)$ uma carta de $M$, temos

$$
\partial_{i}\left(Y^{k}\right) \frac{d \gamma^{i}}{d t}=\frac{\partial\left(Y^{k} \circ \psi\right)}{\partial x_{i}} \cdot \frac{d\left(x^{i} \circ \gamma\right)}{d t}=\frac{d}{d t} Y^{k}
$$

\subsection{Geodésicas}

Definição 2.57. Sejam $\gamma: I \subset \mathbb{R} \rightarrow M$ uma curva em $M$ e $X$ um campo ao longo da curva $\gamma$. O campo $X$ é dito paralelo se

$$
\frac{D}{d t} X_{\gamma(t)}=0
$$

para todo $t \in I$.

Definição 2.58. Sejam $M$ uma variedade semi-Riemanniana de dimensão finita $n e$ $\gamma: I \subset \mathbb{R} \rightarrow M$ uma curva em $M$. Tal curva é dita uma geodésica se o campo tangente é paralelo, ou seja,

$$
\frac{D}{d t} \gamma^{\prime}(t)=0
$$

para todo $t \in I$.

Dessa forma,usando as equações 2.7 e 2.5

$$
\frac{D}{d t} \gamma^{\prime}(t)=\nabla_{\gamma^{\prime}(t)} \gamma^{\prime}(t)=\sum_{k=1}^{n}\left\{\frac{d^{2} \gamma^{k}}{d t^{2}}(t)+\sum_{i, j=1}^{n} \frac{d \gamma^{j}}{d t}(t) \frac{d \gamma^{i}}{d t}(t) \Gamma_{i j}^{k}(t)\right\} \partial_{k}(t)
$$


Vamos denotar

$$
A_{k}(t)=\frac{d^{2} \gamma^{k}}{d t^{2}}(t)+\sum_{i, j=1}^{n} \frac{d \gamma^{j}}{d t}(t) \frac{d \gamma^{i}}{d t}(t) \Gamma_{i j}^{k}(t),
$$

sendo $\gamma^{i}=\varphi^{i} \circ \gamma$, onde $\varphi=\left(\varphi^{1}, \cdots, \varphi^{n}\right)$ é um sistema de coordenadas de $M$. Portanto $\gamma$ é uma geodésica se, e somente se, $A_{k} \equiv 0$, para qualquer $1 \leq k \leq n$. Note que $A_{k}(t)=0$ gera um sistema de equações de $2^{\underline{a}}$ ordem. Logo, fixadas as condições iniciais, segue pelo Teorema de Existência e Unicidade que existe uma única curva geodésica com as condições definidas. Isso prova o seguinte resultado.

Proposição 2.59. Sejam $p \in M$ e $v \in \mathrm{T}_{p} M$, então existe um intervalo $I \ni 0$ e uma única geodésica $\gamma: I \rightarrow M$ tal que $\gamma(0)=p$ e $\gamma^{\prime}(0)=v$.

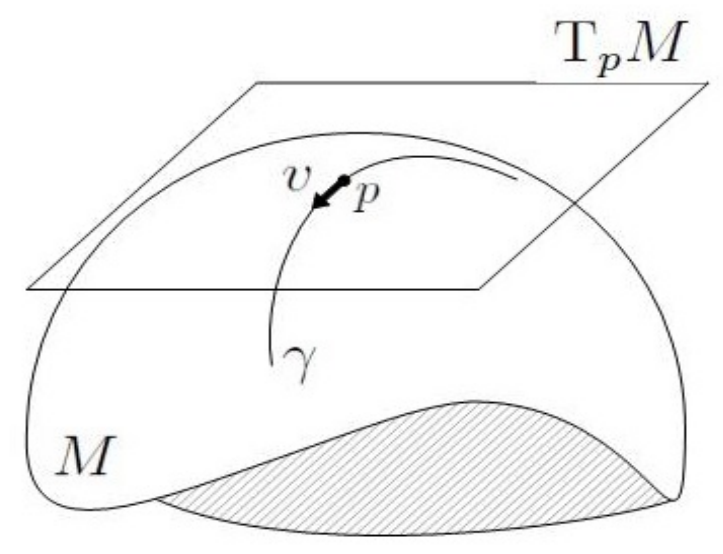

Figura 2.1: Geodésica $\gamma$ com condições iniciais $\gamma(0)=p$ e $\gamma^{\prime}(0)=v$

Exemplo 2.60. Considere a variedade $\mathbb{R}^{n}$ com a conexão $\nabla$ definida no exemplo 2.53. Como visto no exemplo 2.52, os símbolos de Christoffel são todos iguais a zero. Dessa forma, as equações geodésicas 2.10 ficam escritas como

$$
\frac{d^{2} \gamma^{i}}{d t^{2}}=0
$$

para todo $1 \leq i \leq n$, sendo $\gamma^{i}=\varphi^{i} \circ \gamma$. Então $\varphi^{i}(\gamma(t))=p^{i}+t v^{i}$, para todo $t$, onde $p^{i}, v^{i} \in \mathbb{R}$, para todo $i=1, \cdots, n$. Portanto, $\gamma(t)=p+$ tv e as geodésicas de $\mathbb{R}^{n}$ são retas. $E$, em particular, $\mathbb{R}^{n}$ é geodesicamente completa.

Considerando uma curva $\gamma: I \rightarrow M$ qualquer, então $\gamma^{\prime}(t) \in \mathrm{T}_{\gamma(t)} M$ para todo $t \in I$. Sendo $g$ uma métrica semi-Riemanniana então podemos determinar o caráter causal de cada vetor $\gamma^{\prime}(t)$. Entretanto é possível que o caráter causal dos vetores tangentes não sejam iguais pelo caminho da curva, porém quando lidamos com uma geodésica podemos garantir essa constância.

Proposição 2.61. Seja $(M, g)$ uma variedade semi-Riemanniana munida de uma métrica g. Considere $\nabla$ a conexão de Levi-Civita associada a g e $\gamma: I \subset \mathbb{R} \rightarrow M$ uma geodésica em $M \operatorname{com} \gamma^{\prime}$ não identicamente nulo. Então $g\left(\gamma^{\prime}(t), \gamma^{\prime}(t)\right)$ é constante em todo $t \in I$.

Demonstração. Seja $X=\gamma^{\prime}(t)$ o campo tangente ao longo de $\gamma$, então, pelo Teorema 2.52,

$$
X g\left(\gamma^{\prime}(t), \gamma^{\prime}(t)\right)=2 g\left(\frac{D}{d t} \gamma^{\prime}(t), \gamma^{\prime}(t)\right)=0
$$


onde a última igualdade vem do fato de que $\gamma$ é geodésica. Portanto $g\left(\gamma^{\prime}(t), \gamma^{\prime}(t)\right)$ é constante.

Dessa forma, como provado acima, podemos atribuir um caráter causal para geodésicas dependendo do caráter causal de qualquer um dos vetores tangentes da curva. De forma semelhante à Proposição 2.59 podemos enunciar o seguinte lema:

Lema 2.62. Seja $v$ um vetor tangente de $M$, ou seja, $v \in \mathrm{T} M$. Então existe uma vizinhança $\mathcal{U}$ de $v$ em $\mathrm{T} M$ e um intervalo $I \subset \mathbb{R}$ ao redor de 0 tal que $(w, t) \mapsto \gamma_{w}(t)$ é uma função suave bem definida de $\mathcal{U} \times I$ em $M$.

Vimos acima que as curvas geodésicas de uma variedade são soluções de um sistema de equações diferenciais de segunda ordem, o que muitas vezes não pode ser resolvido de modo simples. Entretanto, é conveniente em alguns momentos tomar a curva tangente $\gamma^{\prime}$ e assim reduzir o sistema para equações de primeiro grau. Isso traz algumas vantagens, pois existe um campo vetorial em TM tal que a curva tangente $\gamma^{\prime}$ é uma curva integral desse campo e assim existe uma corresponência entre curvas integrais de campo e geodésicas de $M$.

Definição 2.63. Seja $v \in \mathrm{T} M$ e denote por $G_{v}$ o vetor velocidade inicial da curva $s \mapsto \gamma_{v}^{\prime}(s)$ em $\mathrm{T} M$. Então, definimos o campo vetorial $G \in \mathfrak{X}(\mathrm{T} M)$ como $G: \mathrm{T} M \rightarrow \mathrm{T}(\mathrm{T} M)$ dado por $v \mapsto G_{v}$.

O Lema 2.62 mostra que o campo $G$ é um campo vetorial suave no fibrado TM.

Proposição 2.64. O campo vetorial $G$ é tal que a projeção $\pi: \mathrm{T} M \rightarrow M$ é uma bijeção entre curvas integrais de $G$ e geodésicas de $M$.

Demonstração. (a.) Se $\gamma$ é uma geodésica em $M$, então $\gamma^{\prime}$ é uma curva integral de $G$.

Denotemos $\alpha(s)=\gamma^{\prime}(s)$, para todo $s$. Iremos mostrar que em cada ponto de $\alpha$ o vetor tangente é um elemento do campo $G$. Para isso, fixe $t$ qualquer e sejam $w=\gamma^{\prime}(t)$ e $\beta(s)=\gamma_{w}^{\prime}(s)$; note que $\gamma(t+s)=\gamma_{w}(s)$. Então, tomando velocidades da perspectiva de $M, \alpha(t+s)=\gamma_{w}^{\prime}(s)=\beta(s)$ e, tomando velocidades da perspectiva de $\mathrm{T} M, \alpha^{\prime}(t+s)=\beta^{\prime}(s)$. Em particular,

$$
\alpha^{\prime}(t)=\beta^{\prime}(0)=G_{w}=G_{\alpha(t)},
$$

ou seja, todos os vetores tangentes de $\alpha$ são elementos do campo $G$. Portanto, $\alpha$ é curva integral de $G$.

(b.) Se $\alpha$ é uma curva integral de $G$, então $\pi \circ \alpha$ é uma geodésica em $M$.

Seja $v=\alpha(s)$, tome a geodésica $\gamma_{v}$ e considere a curva $s \mapsto \gamma_{v}^{\prime}(s)$, então, pela unicidade de curvas integrais, temos que, ao menos localmente, $\pi \circ \alpha=\pi \circ \gamma_{v}^{\prime}=\gamma_{v}$. De modo semelhante, considere agora um $t$ arbitrário e seja $\rho$ uma curva integral de $G$ iniciando em $\alpha(t)$. Então, $\alpha(t+s)=\rho(s)$ e portanto $\pi \alpha(t+s)=\pi \rho(s)=\gamma_{\rho(0)}(s)$.

Lema 2.65. Sejam $\gamma: I \rightarrow M$ uma geodésica não constante e $h: J \rightarrow I$ uma função com $J \subset \mathbb{R}$. Uma reparametrização $\gamma \circ h: J \rightarrow M$ é uma geodésica se, e somente se, $h$ é definido da seguinte forma $h(t)=a t+b$.

Demonstração. Para qualquer curva $\gamma$ temos

$$
(\gamma \circ h)^{\prime}(t)=\left(\frac{d h}{d t}\right)(t) \cdot \gamma^{\prime}(h(t)) .
$$

Então:

$$
\frac{D}{d t}(\gamma \circ h)^{\prime}=\frac{d^{2} h}{d t^{2}} \cdot \gamma^{\prime}+\left(\frac{d h}{d t}\right)^{2}(t) \cdot \frac{D}{d t} \gamma^{\prime} .
$$


Por hipótese, $\gamma$ é uma geodésica, $\log o \frac{D}{d t} \gamma^{\prime}=0$ e como $\gamma$ é não constante então $\gamma^{\prime}$ não é identicamente nula.

Portanto,

$$
\gamma \circ h \text { : geodésica } \Longleftrightarrow \frac{D}{d t} \gamma \circ h^{\prime}=0 \Longleftrightarrow \frac{d^{2} h}{d t^{2}}=0 \Longleftrightarrow h(t)=a t+b .
$$

\subsection{Aplicação exponencial}

Definição 2.66. Sejam $p \in M$ e $\mathcal{W}_{p}$ um conjunto de vetores $v$ em $\mathrm{T}_{p} M$ tal que a geodésica $\gamma_{v}$ é definida ao menos em $[0,1]$. A aplicação exponencial de $M$ em $p$ é a função:

$$
\exp _{p}: \mathcal{W}_{p} \rightarrow M
$$

definida por $\exp _{p}(v)=\gamma_{v}(1)$.

Note que $\mathcal{W}_{p}$ é o maior subconjunto de $\mathrm{T}_{p} M$ onde $\exp _{p}$ pode ser definido; de modo que, se $M$ é geodesicamente completo, então $\mathcal{W}_{p}=\mathrm{T}_{p} M$, para todos os pontos $p \in M$.

Do modo como definimos a exponencial a imagem de exp é sempre o ponto da geodésica no instante 1, mas é razoável pensar que existem geodésicas que estão definidas em intervalos maiores do que $[0,1]$, de modo que podemos expandir a exponencial nos limites de definição das geodésicas em um ponto fixado. Para fazer isso, vamos fixar um vetor $v \in \mathrm{T}_{p} M$ e um instante $t \in \mathbb{R}$. Considere a geodésica $\gamma_{v}$ e tome a seguinte reparametrização $s \mapsto \gamma_{v}(t s)$, que também é geodésica pelo Lema 2.65, essa reparametrização tem velocidade inicial

$$
\left.\frac{d}{d s}\left(\gamma_{v}(t s)\right)\right|_{s=0}=t \gamma_{v}^{\prime}(0)=t v
$$

Dessa forma, $\gamma_{t v}(s)=\gamma_{v}(t s)$ pela unicidade da geodésica, como visto no Lema 2.62.

Portanto, se $v \in \mathcal{W}_{p}$ então

$$
\exp _{p}(t v)=\gamma_{t v}(1)=\gamma_{v}(t)
$$

Então a exponencial $\exp _{p}$ leva retas através de $0_{p}$ de $\mathrm{T}_{p} M$ em geodésicas em $M$ passando por $p$.

Proposição 2.67. Para todo $p \in M$ existe uma vizinhança $\tilde{U} \ni 0_{p}$ em $\mathrm{T}_{p} M$ na qual a exponencial $\exp _{p}$ é um difeomorfismo em uma vizinhança $U \ni p$ em $M$.

Demonstração. Pelo Lema 2.62 sabemos que $\exp _{p}$ é uma aplicação bem definida e suave de uma vizinhança de $0_{p}$ em $\mathrm{T}_{p} M$. Afirmamos que a diferencial

$$
d \exp _{p}: \mathrm{T}_{0_{p}}\left(\mathrm{~T}_{p} M\right) \rightarrow \mathrm{T}_{p} M
$$

é um isomosrfismo canônico $v_{0} \mapsto v$. Sabemos que os vetores de $\mathrm{T}_{0_{p}}\left(\mathrm{~T}_{p} M\right)$ são definidos como $v_{0}=\rho^{\prime}(0)$, onde $\rho(t)=t v$, para algum $v \in \mathrm{T}_{p} M$; e como visto antes $\exp _{p}(t v)=\gamma_{v}(t)$. Assim,

$$
d \exp _{p}\left(v_{0}\right)=d \exp _{p}\left(\rho^{\prime}(0)\right)=\left(\exp _{p} \circ \rho\right)^{\prime}(0)=\gamma_{v}^{\prime}(0)=v .
$$

Portanto, pelo Teorema da Função Inversa $\exp _{p}$ é um difeomorfismo local. 


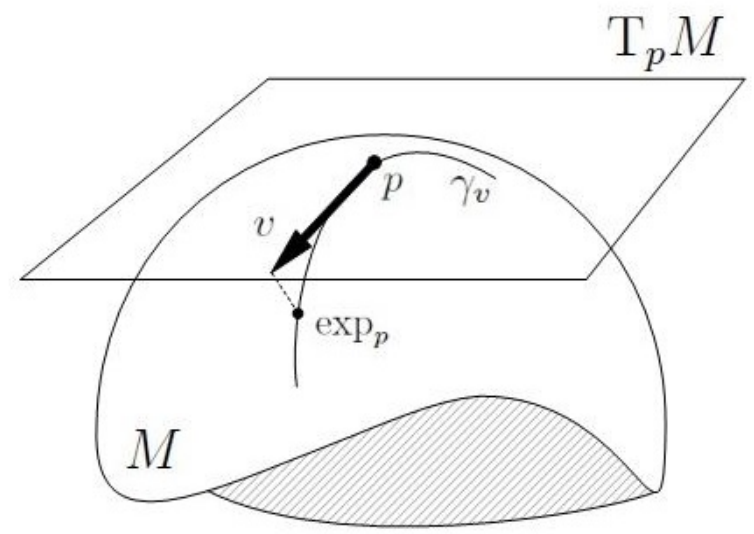

Figura 2.2: Aplicação da exponencial em $\mathrm{T}_{p} M, \exp _{p}(v)=\gamma_{v}(1)$

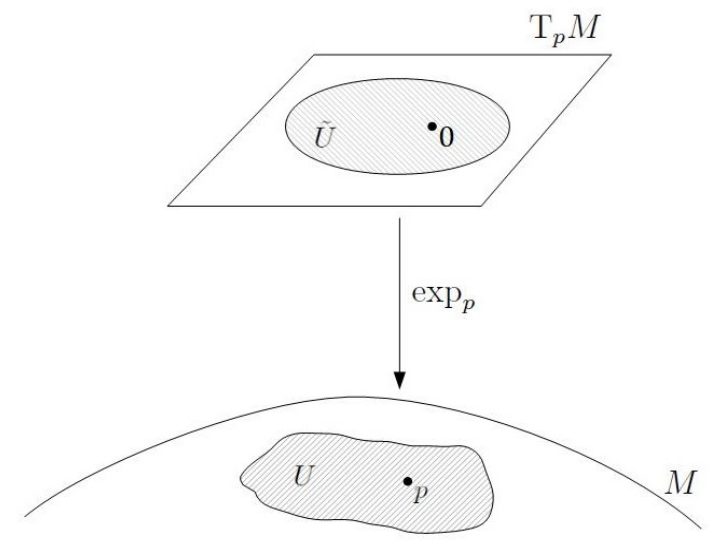

Figura 2.3: A exponecial é um difeomorfismo de $\tilde{U} \in \mathrm{T}_{p} M$ em $U \in M$

\subsection{Campo de Killing}

Definição 2.68. Seja $X$ um campo vetorial em uma variedade $M$. Dizemos que $X$ é um campo vetorial completo se estiver definido em todos os pontos de $M$.

Definição 2.69. O fluxo de um campo vetorial completo $X$ em $M$ é uma aplicação $\psi: M \times \mathbb{R} \rightarrow M$ definida por

$$
\psi(p, t)=\alpha_{p}(t)
$$

onde $\alpha_{p}$ é a curva integral tal que $\alpha_{p}(0)=p$ e $\alpha_{p}^{\prime}(0)=X_{p}$.

Observação 2.70. O fluxo também pode ser definido localmente, dependendo de onde o campo vetorial está definido. Suponha que o campo $X$ esteja definido em uma região $U$ de $M$, logo o fluxo de $U$ é uma aplicação $\psi: U \times I \rightarrow U$, onde $I$ é um intervalo em $\mathbb{R}$ tal que $0 \in I$.

Se fixarmos $p$, então $t \mapsto \psi(p, t)$ é a curva integral $\alpha_{p}$. E caso fixarmos $t$, então $p \mapsto \psi(p, t)$ define uma função $\psi_{t}: M \rightarrow M$ chamada de estágio $t$ do fluxo que leva todo ponto $p \in M$ no correspondente através do fluxo no instante $t$. 


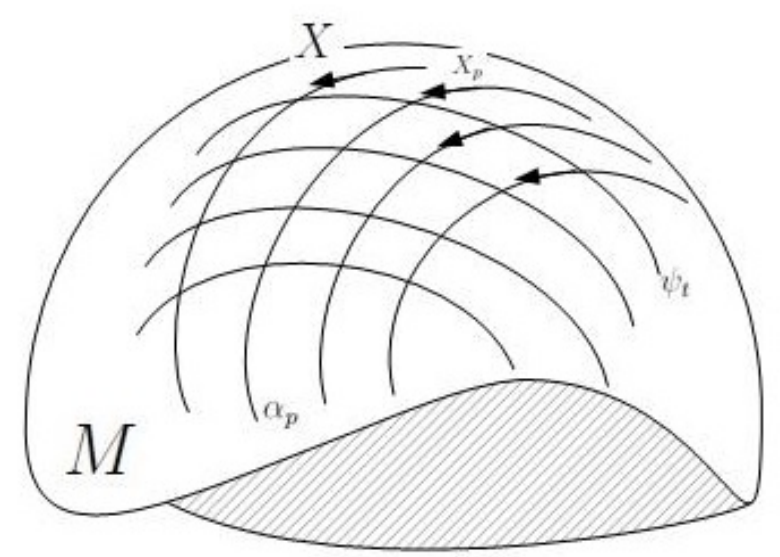

Figura 2.4: Estágios t do fluxo $\psi$

Lema 2.71. Se $\psi$ é o fluxo de um campo vetorial completo, então:

i. $\psi_{0}$ é a função identidade de $M$;

ii. $\psi_{s} \circ \psi_{t}=\psi_{s+t}$, para todo $s, t \in \mathbb{R}$;

iii. cada estágio $\psi_{t}$ é um difeomorfismo, com $\psi_{t}^{-1}=\psi_{-t}$.

Demonstração. $\quad i$. Diretamente da definição, $\psi_{0}(p)=\alpha_{p}(0)=p$.

ii. Para $t$ fixado, seja $\alpha_{p}(t)=q \in M$, note que $\left(\psi_{s} \circ \psi_{t}\right)(p)=\psi_{s}\left(\alpha_{p}(t)\right)=\psi_{s}(q)=\beta_{q}(s)$, onde $\beta_{q}$ é a curva integral tal que $\beta_{q}(0)=q=\alpha_{p}(t)$ e $\beta_{q}^{\prime}(0)=X_{q}=X_{\alpha_{p}(t)}$. Assim, pela unicidade da curva integral, temos que $\alpha_{p}$ e $\beta_{q}$ parametrizam a mesma curva e $\beta_{q}(s)=\alpha_{p}(t+s)$. Portanto, segue o resultado.

iii. Seja $\psi_{t}$ um estágio de um fluxo. A função $\psi_{-t}(p)=\alpha_{p}(-t)$ é um estágio do fluxo. O resultado vem diretamente do item anterior, pois $\psi_{-t} \circ \psi_{t}(p)=\psi_{0}(p)=\alpha_{p}(0)=p$.

Proposição 2.72. Seja $\alpha:[0, b[\rightarrow M$, com $b<\infty$, uma curva integral de $X \in \mathfrak{X}(M)$. Se existe uma sequência $\left(s_{n}\right) \in\left[0, b\left[, \operatorname{com}\left(s_{n}\right) \rightarrow b\right.\right.$ tal que $\left(\alpha\left(s_{n}\right)\right)$ converge, então $\alpha$ é extendivel como curva integral em $[0, b+\delta[$, para algum $\delta$.

Demonstração. Seja $U$ uma vizinhança de $L=\lim \alpha\left(s_{n}\right)$ tal que o fluxo esteja definido $U \times]-\delta, \delta\left[\right.$, para algum $\delta$. Como $\left(s_{n}\right) \rightarrow b$, existe $n_{0}$ tal que $\left|s_{n_{0}}-b\right|<\delta$, então $s_{n_{0}}-b>-\delta$ e $s_{n_{0}}>b-\delta$, e ainda $\alpha\left(s_{n_{0}}\right) \in U$, devido a convergência de $\left(\alpha\left(s_{n}\right)\right)$.

Tomemos então $\beta$ uma curva integral de $X$ passando por $\alpha\left(s_{n_{0}}\right)$ e definida em $[0, \delta[$. Portanto, $\beta(t)=\alpha\left(s_{n_{0}}+t\right)$ e $\beta$ é a extensão desejada.

Definição 2.73. Seja $X \in \mathfrak{X}(M)$. A derivada de Lie $L_{X}$ relativa a $X$ é definida por

$$
\begin{gathered}
L_{X}(f)=X f \\
L_{X}(Y)=[X, Y]
\end{gathered}
$$

para quaisquer $f \in \mathcal{F}(M)$ e $Y \in \mathfrak{X}(M)$.

Na Definição anterior vimos como está determinada a derivada de Lie quando aplicada em funções e campos vetoriais. Uma definição similar pode ser feita a um campo tensorial covariante $A$. 
Definição 2.74. Sejam $X \in \mathfrak{X}(M)$ e $A \in \mathfrak{T}_{s}(M)$, um $s$-campo tensorial covariante. $A$ derivada de Lie $L_{X}$ de $A$ relativa a $X$ é definida por

$$
L_{X} A=\lim _{t \rightarrow 0} \frac{1}{t}\left[\psi_{t}^{*}(A)-A\right] .
$$

A derivada de Lie é um caso particular de derivação tensorial e portanto vale a regra do produto:

$$
L_{X} A(Y, Z)=X A(Y, Z)-A([X, Y], Z)-A([X, Z], Y),
$$

aqui mostrado para o caso $s=2$. Para estudos mais aprofundados em tensores e derivadas de tensores deixamos indicado [O'N83] e [KN63].

Definição 2.75. Um campo vetorial de Killing em uma variedade é um campo vetorial $X$ tal que a derivada de Lie aplicada a métrica da variedade $g$ é nula, ou seja, $L_{X} g=0$.

Proposição 2.76. Um campo vetorial $X$ é de Killing se, e somente se, os estágios $\psi_{t}$ de todos os fluxos locais são isometrias.

Demonstração. Se cada $\psi_{t}$ é uma isometria, então $\psi_{t}^{*}(g)=g$. Portanto, pela Definição 2.74, $L_{X} g=0$.

Reciprocamente, se $L_{X} g=0$, seja $\psi: U \times I \rightarrow U$ um fluxo local de $X$. Se $v \in \mathrm{T}_{p} M$, sendo $p \in U$, então, para $s$ suficientemente pequeno, $w=d \psi_{s}(v)$ e, pela Definição,

$$
\lim _{t \rightarrow 0} \frac{1}{t}\left[g\left(d \psi_{t} w, d \psi_{t} w\right)-g(w, w)\right]=0
$$

E como $\psi_{s} \circ \psi_{t}=\psi_{s+t}$,

$$
\lim _{t \rightarrow 0} \frac{1}{t}\left[g\left(d \psi_{s+t}(v), d \psi_{s+t}(v)\right)-g\left(d \psi_{s}(v), d \psi_{s}(v)\right)\right]=0 .
$$

Dessa forma, a função a valores reais $s \mapsto g\left(d \psi_{s}(v), d \psi_{s}(v)\right)$ tem derivada identicamente nula. Portanto, a função é constante e $\operatorname{assim} g\left(d \psi_{s}(v), d \psi_{s}(v)\right)=g(v, v)$, para todo $v$ e $s \in I$.

Proposição 2.77. Se $\nabla$ é uma conexão de Levi-Civita e $X$ é um campo de Killing, então $\left\langle\nabla_{Y} X, Z\right\rangle+\left\langle\nabla_{Z} X, Y\right\rangle=0$, para quaisquer $Y, Z \in \mathfrak{X}(M)$

Demonstração. Como $X$ é um campo vetorial de Killing, então pela equação 2.11

$$
\begin{array}{r}
X\langle Y, Z\rangle=\langle[X, Y], Z\rangle+\langle Y,[X, Z]\rangle=\left\langle\nabla_{X} Y-\nabla_{Y} X, Z\right\rangle+\left\langle Y, \nabla_{X} Z-\nabla_{Z} X\right\rangle \\
=\left\langle\nabla_{X} Y, Z\right\rangle-\left\langle\nabla_{Y} X, Z\right\rangle+\left\langle\nabla_{X} Z, Y\right\rangle-\left\langle\nabla_{Z} X, Y\right\rangle \\
=X\langle Y, Z\rangle-\left(\left\langle\nabla_{Y} X, Z\right\rangle+\left\langle\nabla_{Z} X, Y\right\rangle\right) .
\end{array}
$$

Portanto, $\left\langle\nabla_{Y} X, Z\right\rangle+\left\langle\nabla_{Z} X, Y\right\rangle=0$.

Lema 2.78 (Lema de conservação). Sejam $X$ um campo de Killing em $M$ e $\gamma$ uma geodésica em $M$. Então $\left\langle\gamma^{\prime}, X\right\rangle$ é constante ao longo de $\gamma$.

Demonstração. Seja $\nabla$ a conexão de Levi-Civita associada a métrica $g$. Pela Proposição 2.77 e considerando $Y=Z=\gamma^{\prime}$, teremos $\left\langle\nabla_{\gamma^{\prime}} X, \gamma^{\prime}\right\rangle=0$. Portanto,

$$
\frac{d}{d t}\left\langle X_{\gamma}, \gamma^{\prime}\right\rangle=\left\langle\frac{D}{d t} X_{\gamma}, \gamma^{\prime}\right\rangle+\left\langle X_{\gamma}, \frac{D}{d t} \gamma^{\prime}\right\rangle=\left\langle\nabla_{\gamma^{\prime}} X_{\gamma}, \gamma^{\prime}\right\rangle=0
$$




\subsection{Relação entre formas bilineares e produtos escalares}

Nas seções anteriores foi discutido o impacto que um produto escalar pode ter na base do espaço vetorial em que está definido. Além disso foi mencionado que os resultados determinavam a necessidade de fixar um produto escalar e que os vetores das bases e os tipos de vetores poderiam variar dependendo do produto escalar considerado no espaço vetorial. Veremos agora um resultado que exibe uma certa relação entre um produto escalar e uma forma bilinear simétrica sob certas condições.

Teorema 2.79. Sejam $(V, g)$ sendo g um produto escalar indefinido, com $\operatorname{dim} V=n$ e $b$ uma forma bilinear simétrica. Então, são equivalentes:

i. $b=\lambda \cdot g$, para algum $\lambda \in \mathbb{R}$;

ii. $q_{b}(v)=0$, para todo $v \in V$ tal que $g(v, v)=0$.

Demonstração. (i. $\Rightarrow$ ii.) Suponha que $b=\lambda \cdot g$, para algum $\lambda \in \mathbb{R}$. Seja $v \in V$ um vetor tipo-luz de $g$. Então

$$
q_{b}=b(v, v)=\lambda \cdot g(v, v)=0
$$

portanto vale o resultado.

$($ ii. $\Rightarrow$ i.) Vamos dividir em casos.

- Caso I: $n=2, \nu=1$. Seja $\mathcal{B}$ uma base ortonormal de $V, \mathcal{B}=\left\{e_{1}, e_{2}\right\}$, sobre $g$. Sem perda de generalidade, suponha que $e_{2}$ é tipo-tempo de $g$, pois $\nu=1$. Definimos os vetores

$$
v_{1}=\frac{1}{\sqrt{2}}\left(e_{1}+e_{2}\right) \quad \text { e } \quad v_{2}=\frac{1}{\sqrt{2}}\left(e_{1}-e_{2}\right) .
$$

Então $v_{1}$ e $v_{2}$ são tipo-luz de $g$. De fato,

$$
g\left(v_{1}, v_{1}\right)=\frac{1}{2}\left[g\left(e_{1}, e_{1}\right)+2 g\left(e_{1}, e_{2}\right)+g\left(e_{2}, e_{2}\right)\right]=0
$$

$\mathrm{e}$

$$
g\left(v_{2}, v_{2}\right)=\frac{1}{2}\left[g\left(e_{1}, e_{1}\right)+g\left(e_{2}, e_{2}\right)\right]=0 .
$$

Logo, como são tipo-luz de $g$, por hipótese, também são vetores tipo-luz de $b$. Note que $v_{1}$ e $v_{2}$ formam uma base de $V$, seja $\tilde{\mathcal{B}}=\left\{v_{1}, v_{2}\right\}$ uma base e tome $\lambda=b\left(v_{1}, v_{2}\right)$. Dessa forma teremos

$$
M_{\tilde{\mathcal{B}}}(b)=\left[\begin{array}{ll}
b\left(v_{1}, v_{1}\right) & b\left(v_{1}, v_{2}\right) \\
b\left(v_{2}, v_{1}\right) & b\left(v_{2}, v_{2}\right)
\end{array}\right]=\left[\begin{array}{cc}
0 & \lambda \\
\lambda & 0
\end{array}\right]=\lambda \cdot\left[\begin{array}{cc}
0 & 1 \\
1 & 0
\end{array}\right] .
$$

e ainda

$$
M_{\tilde{\mathcal{B}}}(g)=\left[\begin{array}{ll}
0 & 1 \\
1 & 0
\end{array}\right]
$$

Portanto, $b=\lambda \cdot g$.

- Caso II: $n>2, \nu=1$. Seja $\mathcal{B}=\left\{e_{1}, \ldots, e_{n}\right\}$ uma base ortonormal com respeito a $g$ com $e_{n}$ tipo-tempo e $b_{i j}=b\left(e_{i}, e_{j}\right)$. Considere os subespaços $W_{i}=\operatorname{span}\left(e_{i}, e_{n}\right)$, para $i=1, \ldots, n-1$. Aplicando o Caso I em $W_{i}$ teremos que $b_{i n}=0$ e $b_{i i}=-b_{n n}$, para todo 
$i=1, \ldots, n-1$, isso acontece pois em $W_{i}$ vale que $b=\lambda \cdot g$. Logo, sendo $b_{n n}=-\lambda$

$$
M_{\mathcal{B}}(b)=\left[\begin{array}{ccc|c}
\lambda & \cdots & * & 0 \\
\vdots & \ddots & \vdots & \vdots \\
* & \cdots & \lambda & 0 \\
\hline 0 & \cdots & 0 & -\lambda
\end{array}\right]
$$

Definimos os vetores $v_{\theta}=\cos (\theta) e_{i}+\sin (\theta) e_{j}+e_{n}$, para quaisquer $i, j>1, i \neq j ; v_{\theta}$ é do tipo-luz sobre $g$, para qualquer $\theta$.

$$
\begin{array}{r}
g\left(v_{\theta}, v_{\theta}\right)=\cos ^{2}(\theta) g\left(e_{i}, e_{i}\right)+\sin ^{2}(\theta) g\left(e_{j}, e_{j}\right)+2 \sin (\theta) \cos (\theta) g\left(e_{i}, e_{j}\right)+g\left(e_{n}, e_{n}\right) \\
=\sin (2 \theta) g\left(e_{i}, e_{j}\right)=0 .
\end{array}
$$

Logo, $v_{\theta}$ é tipo-luz para $b$ (hipótese). Então segue que

$$
0=b\left(v_{\theta}, v_{\theta}\right)=\sin (2 \theta) b_{i j}
$$

assim temos que $b_{i j}=0$, para quaisquer $i, j=1, \ldots$, e $i \neq j$; portanto

$$
M_{\mathcal{B}}(b)=\left[\begin{array}{ccc|c}
\lambda & \cdots & 0 & 0 \\
\vdots & \ddots & \vdots & \vdots \\
0 & \cdots & \lambda & 0 \\
\hline 0 & \cdots & 0 & -\lambda
\end{array}\right]=\lambda \cdot\left[\begin{array}{ccc|c}
1 & \cdots & 0 & 0 \\
\vdots & \ddots & \vdots & \vdots \\
0 & \cdots & 1 & 0 \\
\hline 0 & \cdots & 0 & -1
\end{array}\right]=\lambda \cdot M_{\mathcal{B}}(g) .
$$

- Caso III: $n \geq 2, \nu=n-1$. Se $g$ tem índice $n-1$ então a forma bilinear $-g$ tem índice 1. Aplicando o Caso II para $-g$ teremos que $b=\lambda \cdot(-g)$, portanto $b=-\lambda \cdot g=\kappa \cdot g$.

- Caso IV: $n \geq 3,1<\nu<n-1$. Seja $\mathcal{B}=\left\{e_{1}, \ldots, e_{n-\nu}, e_{n-\nu+1}, \ldots, e_{n}\right\}$ base ortonormal sobre $g$, com $e_{n-\nu+1}, \ldots, e_{n}$ vetores do tipo-tempo. Com $b_{i j}=b\left(e_{i}, e_{j}\right)$ e tomando o subespaço $W_{k}=\operatorname{span}\left(e_{1}, \ldots, e_{n-\nu}, e_{k}\right)$ com $k=n-\nu+1, \ldots, n$, pelo Caso II, temos $b_{k k}=-\lambda$ e $b_{i i}=\lambda$, para $i=1, \ldots, n-\nu$. Então

$$
M_{\mathcal{B}}(b)=\left[\begin{array}{c|ccc}
\lambda I_{n-\nu} & 0 & \cdots & 0 \\
\hline 0 & -\lambda & \cdots & * \\
\vdots & \vdots & \ddots & \vdots \\
0 & * & \cdots & -\lambda
\end{array}\right]
$$

Onde $I_{n-\nu}$ é a matriz identidade de ordem $n-\nu$. Considerando os subespaços $V_{k}=\operatorname{span}\left(e_{k}, e_{n-\nu+1}, \ldots, e_{n}\right), \operatorname{com} k=1, \ldots, n-\nu$. Aplicando o Caso III concluímos a demonstração, pois

$$
M_{\mathcal{B}}(b)=\left[\begin{array}{c|c}
\lambda I_{\nu} & 0 \\
\hline 0 & -\lambda I_{n-\nu}
\end{array}\right] .
$$




\section{Capítulo 3}

\section{Espaços vetoriais de Lorentz}

Agora que estudamos as estruturas de espaços vetoriais não-euclidianos, como ortogonalidade e bases, vamos focar no caso Lorentziano, ou seja, espaços com produtos escalares de índice 1. Dessa forma estaremos lidando com espaços cujas bases ortonormais contêm apenas um vetor unitário do tipo-tempo.

Os vetores tipo-tempo exercem um papel muito importante no estudo de espaços nãoeuclidianas. Isso deve ter ficado claro nos resultados vistos anteriormente e será ainda mais evidente nos resultados que veremos a seguir.

Nessa seção iremos denotar por $(V, g)$ um espaço vetorial $V$ de dimensão finita $n$ munido de um produto escalar Lorentziano $g$, isto é, $\nu=1$. Logo, $(V, g)$ é dito um espaço vetorial Lorentziano. Lembrando que o produto escalar pode ser denotado também por $\langle\cdot, \cdot\rangle$.

\subsection{Cones tipo-tempo}

Denotaremos das seguintes formas os conjuntos abaixo:

- $\mathfrak{S}=\{v \in V \mid g(v, v)>0\}$, conjunto dos vetores tipo-espaço de $V$;

- $\mathfrak{T}=\{v \in V \mid g(v, v)<0\}$, conjunto dos vetores tipo-tempo de $V$;

- $\mathfrak{L}=\{v \in V \mid g(v, v)=0\}$, conjunto dos vetores tipo-luz de $V$;

- $\mathfrak{N S}=\{v \in V \mid g(v, v) \leq 0\}$, conjunto dos vetores não tipo-espaço de $V$.

Definição 3.1. Sejam $(V, g)$ um espaço vetorial Lorentziano e $v \in \mathfrak{T}$. Definimos os cones tipo-tempo de $v$ como

- cone tipo-tempo futuro, $C^{\uparrow}(v)=\{w \in \mathfrak{T} \mid g(v, w)<0\}$;

- cone tipo-tempo passado, $C^{\downarrow}(v)=\{w \in \mathfrak{T} \mid g(v, w)>0\}$.

Podemos definir também os cones causais de $v$ :

- $\bar{C}^{\uparrow}(v)=\{w \in \mathfrak{N S} \mid g(v, w)<0\} ;$

- $\bar{C}^{\downarrow}(v)=\{w \in \mathfrak{N S} \mid g(v, w)>0\}$.

Note que, pela Definição acima, $v \in C^{\uparrow}(v)$, enquanto $-v \in C^{\downarrow}(v)$.

A denominação por cone nos conjuntos acima é efeito do caso $\mathbb{L}^{3}$ onde esses conjuntos são, de fato, cones no espaço. Consideremos $v \in \mathbb{L}^{3}$ um vetor qualquer escrito na base canônica como $v=(x, y, z)$. Como estamos tomando o produto escalar $g=d x^{2}+d y^{2}-d z^{2}$ teremos que 
- se $v \in \mathfrak{S}$, então $z^{2}<x^{2}+y^{2}$;

- se $v \in \mathfrak{T}$, então $z^{2}>x^{2}+y^{2}$;

- se $v \in \mathfrak{L}$, então $z^{2}=x^{2}+y^{2}$.

E assim, fica fácil ver que os conjuntos $\mathfrak{S}, \mathfrak{T}$ e $\mathfrak{L}$ são uniões de dois cones.

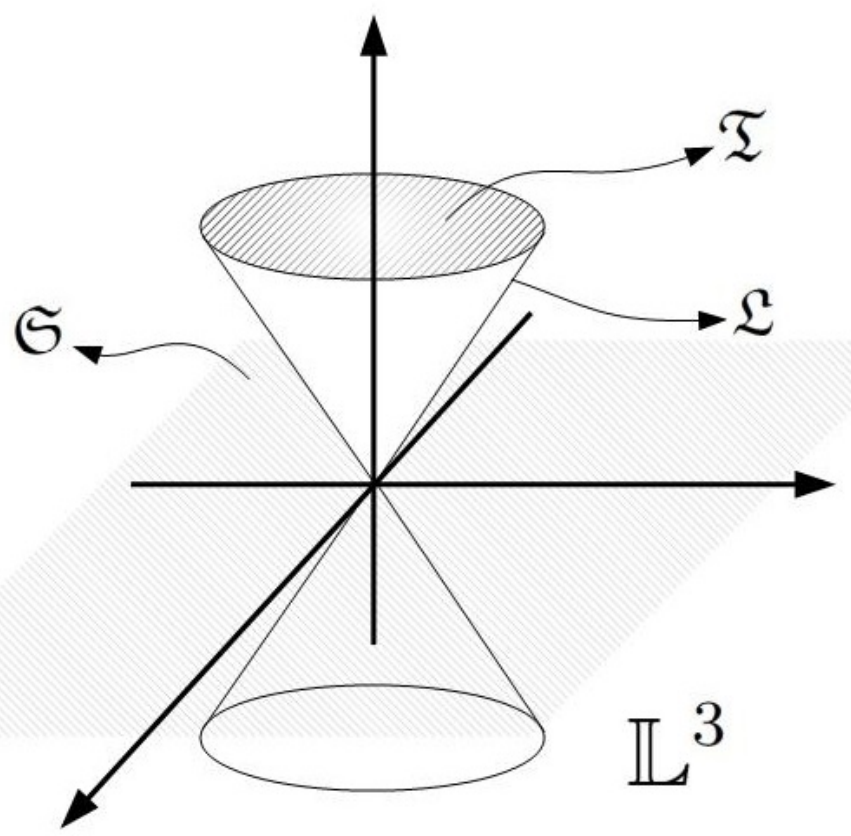

Figura 3.1: Os cones temporais em $\mathbb{L}^{3}$

Nos enunciados a seguir quando não for mencionado ou destacado a orientação temporal (passado ou futuro) do cone é porque os resultados valem independente dos casos.

Proposição 3.2. Dois vetores tipo-tempo $v$ e w estão no mesmo cone tipo-tempo se, e somente se, $g(v, w)<0$.

Demonstração. $(\Rightarrow)$ Por hipótese, $v$ e $w$ estão no mesmo cone tipo-tempo. Então existe $u \in \mathfrak{T}$ tal que $v, w \in C(u) \mathrm{e}$

$$
\begin{aligned}
g(u, v) & <0 \\
g(u, w) & <0
\end{aligned}
$$

Suponhamos, sem perda de generalidade, que $\|u\|=1$. Tome então uma base ortonormal $\left\{e_{1}, \ldots, e_{n-1}, u\right\}$. Logo,

$$
\left\{\begin{array}{c}
v=\alpha u+\vec{v} \\
w=\beta u+\vec{w}
\end{array}\right.
$$

sendo $\alpha, \beta \in \mathbb{R}$, com $\alpha, \beta>0$, pelas equações 3.2 , e $\vec{v}, \vec{w} \in \mathfrak{S}$. Então

$$
\begin{aligned}
& 0>g(v, v)=-\alpha^{2}+g(\vec{v}, \vec{v}) \Rightarrow \alpha>\|\vec{v}\| \\
& 0>g(w, w)=-\beta^{2}+g(\vec{w}, \vec{w}) \Rightarrow \beta>\|\vec{w}\|
\end{aligned}
$$

Dessa forma, temos que

$$
g(v, w)=-\alpha \beta+g(\vec{v}, \vec{w})<-\|\vec{v}|\|\mid \vec{w}\|+g(\vec{v}, \vec{w}) \leq 0,
$$


onde a última desigualdade vem de Cauchy-Schwarz. E portanto, $g(v, w)<0$.

$(\Leftarrow)$ Óbvio, pois se $g(v, w)<0$ então tanto $w \in C(v)$ quanto $v \in C(w)$ e portanto estão no mesmo cone tipo-tempo.

Pela própria Definição 3.1 temos que $v \in C(w)$ se, e somente se, $w \in C(v)$. E de modo análogo ao que foi feita na Proposição anterior temos que $v \in C(w)$ se, e somente se, $C(v)=C(w)$.

Proposição 3.3. Se $v$ e $w$ são vetores tipo-tempo no mesmo cone, então $\alpha v+\beta w$, para quaisquer $\alpha, \beta \in \mathbb{R}$ tais que $\alpha \beta>0$, também está no mesmo cone.

Demonstração. Da Proposição 3.2 temos que $g(v, w)<0$, então

$$
\begin{aligned}
g(v, \alpha v+\beta w) & =\alpha g(v, v)+\beta g(v, w)<0 \\
g(\alpha v+\beta w, \alpha v+\beta w) & =\alpha^{2} g(v, v)+2 \alpha \beta g(v, w)+\beta^{2} g(w, w)<0
\end{aligned}
$$

Da segunda equação deduzimos que $\alpha v+\beta w$ é tipo-tempo e da primeira que $\alpha v+\beta w$ está no mesmo cone tipo-tempo que $v$.

Dessa forma, mostramos que cada cone tipo-tempo é convexo.

\subsection{Desigualdades reversas}

Teorema 3.4 (Desigualde de Cauchy-Schwarz reversa). Se v,w $\in$ V são vetores tipotempo, então

i. $|g(v, w)| \geq\|v\|\|w\|$, além disso a igualdade vale se, e somente se, $v$ e $w$ são colineares.

ii. Se $v$ e w estão no mesmo cone, então existe um único $\theta \geq 0$, chamado de ângulo hiperbólico entre $v$ e $w$ tal que

$$
g(v, w)=-\|v\|\|w\| \cosh (\theta) .
$$

Demonstração. $\quad i$. Sabemos que $(V, g)$ é Lorentziano, $\operatorname{logo} \nu=1$. Por hipótese $v$ é tipotempo, então $\operatorname{span}(v)$ é não degenerado, dessa forma $V=\operatorname{span}(v) \oplus \operatorname{span}(v)^{\perp}$, pela Proposição 2.21. Sejam $\alpha \in \mathbb{R}$ e $\bar{w} \in \operatorname{span}(v)^{\perp}$ tais que $w=\alpha v+\bar{w}$. Então

$$
g(w, w)=\alpha^{2} g(v, v)+g(\bar{w}, \bar{w})
$$

Note que $\operatorname{span}(v)^{\perp}$ não tem vetores tipo-tempo, caso contrário, se em sua base houvessem vetores tipo-tempo, então o índice de $V$ seria maior que 1. Então $g(\bar{w}, \bar{w}) \geq 0$. Assim, temos que

$$
\begin{aligned}
g(v, w)^{2}=\alpha^{2} g(v, v)^{2}=g(v, v)[g(w, w)-g(\bar{w}, \bar{w})] \geq g(v, v) g(w, w) \\
=|g(v, v)||g(w, w)|=\|v\|^{2}\|w\|^{2}
\end{aligned}
$$

portanto $|g(v, w)| \geq\|v\|\|w\|$.

É fácil ver que a igualdade vale se, e somente se, $g(\bar{w}, \bar{w})=0$, ou seja, se, e somente se, $v$ e $w$ são colineares. 
ii. Se $v$ e $w$ estão no mesmo cone, então $g(v, w)<0$ (Proposição 3.2); logo, pelo item $i$. vale que

$$
\frac{-g(v, w)}{\|v\|\|w\|} \geq 1
$$

A função cosh definida em $\mathbb{R}$ é tal que $\cosh x \geq 1$, para todo $x \in \mathbb{R}$. Portanto existe $\theta \geq 0$ tal que

$$
\cosh \theta=\frac{-g(v, w)}{\|v\|\|w\|}
$$

Teorema 3.5 (Desigualdade triangular reversa). Se $v$ e w são vetores tipo-tempo no mesmo cone, então

$$
\|v\|+\|w\| \leq\|v+w\|,
$$

e a igualdade vale se, e somente se, $v$ e w são colineares.

Demonstração. Como $v$ e $w$ estão no mesmo cone então $v+w$ é tipo-tempo e $g(v+w, v+w)<0$ (Proposições 3.3 e 3.2, respectivamente). Portanto,

$$
\begin{array}{r}
\|v+w\|^{2}=-g(v+w, v+w)=\|v\|^{2}+\|w\|^{2}+2|g(v, w)| \geq\|v\|^{2}+\|w\|^{2}+2\|v\|\|w\| \\
=(\|v\|+\|w\|)^{2} .
\end{array}
$$

Além disso, a igualdade vale se, e somente se, $|g(v, w)|=\|v\|\|w\|$, ou seja, pelo Teorema 3.4 , se, e somente se, $v$ e $w$ são colineares.

Como destacado em [Ste12], o Teorema 3.5 carrega um valor geométrico muito forte. Na geometria Euclidiana, a distância entre um ponto A e um ponto C não pode ser menor caso passe por um outro ponto B, ou seja, a distância "direta"entre A e C deve ser igual ou menor do que a distância acumulada de $\mathrm{A}$ até $\mathrm{B}$ e de $\mathrm{B}$ até $\mathrm{C}$, isso é a desigualdade triangular euclidiana, tendo esse nome já que na geometria Euclidiana um lado de um triângulo é sempre menor que a soma dos outros dois.

Com a alteração na métrica agora tal propriedade não se mantém e essa diferença pode ser interpretada como uma "curvatura"do espaço, mas não uma curvatura no sentido usual do termo e sim como se houvesse um certo "acúmulo"de espaço, tal ideia traz fortes aplicações físicas e foi um dos fundamentos explorados por Einstein em sua Relatividade Geral.

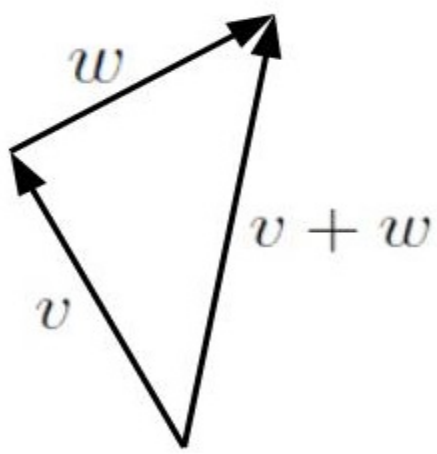

(a)

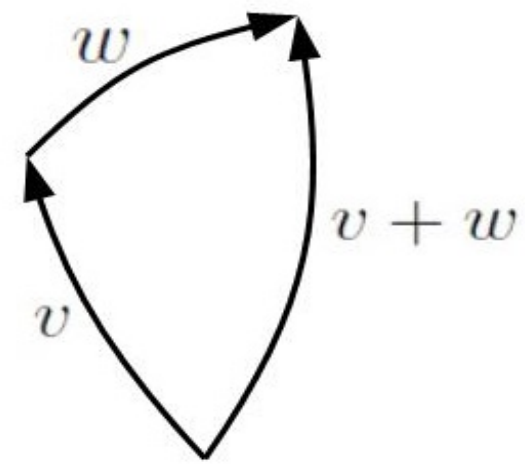

(b)

Figura 3.2: Representação da desigualdade triangular nos casos Euclidiano e não-Euclidiano 


\subsection{Cones causais}

Iremos mostrar propriedades semelhantes àquelas vistas na seção 3.1 para cones tipotempo agora para cones causais.

Proposição 3.6. Se v e w são vetores tipo-luz de um espaço vetorial $V$, então $\{v, w\}$ é linearmente depedente se, e somente se, $g(v, w)=0$.

Demonstração. $(\Rightarrow)$ Se $\{v, w\}$ é linearmente dependente então $w=\lambda v$, para algum $\lambda \in \mathbb{R}$; logo, $g(v, w)=\lambda g(v, v)=0$.

$(\Leftarrow)$ Considere uma base ortonormal para $V,\left\{e_{1}, \cdots, e_{n}\right\}$, então podemos escrever

$$
v=\sum_{i=1}^{n} \alpha^{i} e_{i} \quad \text { e } \quad w=\sum_{j=1}^{n} \beta^{j} e_{j} .
$$

Como $u$ e $w$ são vetores tipo-luz, $\log o \alpha^{n} \neq 0$ e $\beta^{n} \neq 0$. Dessa forma, tomando, sem perda de generalidade, os vetores apropriados teremos que

$$
v=\sum_{i=1}^{n-1} \alpha^{i} e_{i}+e_{n} \quad \text { e } \quad w=\sum_{j=1}^{n-1} \beta^{j} e_{j}+e_{n} .
$$

Por hipótese, $g(v, w)=g(v, v)=g(w, w)=0$, assim

$$
1=\alpha^{1} \beta^{1}+\cdots+\alpha^{n-1} \beta^{n-1}=\left(\alpha^{1}\right)^{2}+\cdots+\left(\alpha^{n-1}\right)^{2}=\left(\beta^{1}\right)^{2}+\cdots+\left(\beta^{n-1}\right)^{2} .
$$

Com isso, pela desigualdade de Cauchy-Schwarz, teremos que os vetores $\alpha^{1} e_{1}+\cdots+\alpha^{n-1} e_{n-1}$ e $\beta^{1} e_{1}+\cdots+\beta^{n-1} e_{n-1}$ são colineares com constante de proporcionalidade 1. Portanto, $v=w$.

Proposição 3.7. Se $v, w \in V$ são vetores causais, então $v$ e w estão no mesmo cone causal se, e somente se, $g(v, w)<0$ ou $v, w \in \mathfrak{L} e\{v, w\}$ é linearmente dependente.

Demonstração. $(\Rightarrow)$ Faremos a demosntração por casos.

- Se $v, w \in \mathfrak{T}$, então o resultado vale pela Proposição 3.2.

- Se algum dos vetores é tipo-luz, então basta tomar o cone causal do outro vetor e o resultado sai imediatamente da observação feita após a Proposição 3.2.

- Se $v, w \in \mathfrak{L}$, então vamos supor que $g(v, w) \geq 0$ e mostraremos que $\{v, w\}$ é linearmente dependente. A demonstração é semelhante àquela da Proposição 3.2. Por hipótese $v$ e $w$ estão no mesmo cone causal, então existe um vetor tipo-tempo $u \in V$ tal que $v, w \in \bar{C}(u)$, ou seja,

$$
\begin{aligned}
g(u, v) & <0 \\
g(u, w) & <0
\end{aligned}
$$

Suponhamos, sem perda de generalidade, que $\|u\|=1$. Tome então uma base ortonormal $\left\{e_{1}, \ldots, e_{n-1}, u\right\}$. Logo,

$$
\left\{\begin{array}{r}
v=\alpha u+\vec{v} \\
w=\beta u+\vec{w}
\end{array}\right.
$$


sendo $\alpha, \beta \in \mathbb{R}$, com $\alpha, \beta>0$, pelas equações 3.2 , e $\vec{v}, \vec{w} \in \mathfrak{S}$. Então

$$
\begin{aligned}
& 0=g(v, v)=-\alpha^{2}+g(\vec{v}, \vec{v}) \Rightarrow \alpha=\|\vec{v}\| \\
& 0=g(w, w)=-\beta^{2}+g(\vec{w}, \vec{w}) \Rightarrow \beta=\|\vec{w}\|
\end{aligned}
$$

Dessa forma, temos que

$$
g(v, w)=-\alpha \beta+g(\vec{v}, \vec{w})=-\|\vec{v}\|\|\vec{w}\|+g(\vec{v}, \vec{w}) \leq 0,
$$

onde a última desigualdade vem da versão euclidiana de Cauchy-Schwarz, válida para vetores tipo-espaço. Temos então que $g(v, w)=0$ e portanto, pela Proposição 3.6, $\{v, w\}$ é linearmente dependente.

$(\Leftarrow)$ Também será feita por casos.

- Se ao menos um dos vetores é tipo-tempo, então o resultado segue ou da Proposição 3.2 ou da observação feita logo após essa proposição.

- Se $v, w \in \mathfrak{L}$ e $\{v, w\}$ é linearmente dependente, então $w=\lambda v$, para algum $\lambda \in \mathbb{R}$, e o resultado é imediato.

Suponha agora que $\{v, w\}$ é linearmente independente e assim $g(v, w)<0$. Então $v+w$ é um vetor tipo-tempo, de fato

$$
g(v+w, v+w)=2 g(v, w)<0 .
$$

Tomemos agora o cone causal $\bar{C}(v+w)$ e note que

$$
\begin{aligned}
g(v+w, v) & =g(w, v)<0 \\
g(v+w, w) & =g(v, w)<0
\end{aligned}
$$

Portanto, $v, w \in \bar{C}(v+w)$ e vale o resultado.

\subsection{Subespaços Lorentzianos}

Definição 3.8. Seja $(V, g)$ um espaço vetorial Lorentziano. Se $W$ é um subespaço vetorial de $V$ então diremos que $W$ é:

i. tipo-espaço, se $\left.g\right|_{W}$ é Euclidiano.

ii. tipo-tempo, se $\left.g\right|_{W}$ é não degenerado com índice 1 (ou seja, Lorentziano caso $\operatorname{dim} W \geq 2$ ).

iii. tipo-luz (ou degenerado), se $\left.g\right|_{W}$ é degenerado (ou seja, $W \cap W^{\perp} \neq\{0\}$ ).

Proposição 3.9. Um subespaço $W$ de um espaço Lorentziano $V$ é tipo-tempo se, e somente se, $W^{\perp}$ é tipo-espaço.

Demonstração. Da Proposição 2.21 segue que $\left.g\right|_{W}$ é não degenerado se, e somente se, $\left.g\right|_{W^{\perp}}$ é não degenerado, ou seja, $W \cap W^{\perp}=\{0\}$. Logo, $V=W \oplus W^{\perp}$ então

$$
\nu_{g}=\nu_{\left.g\right|_{W}}+\nu_{\left.g\right|_{W^{\perp}}}
$$


onde $\nu$ indica os índices da restrições de $g$. Se $W$ é tipo-tempo, então $\nu_{\left.g\right|_{W}}=1$, e se $W^{\perp}$ é tipo-espaço, então $\nu_{\left.g\right|_{W^{\perp}}}=0$. Portanto, como $\nu_{g}=1$, por hipótese, vale o resultado.

Proposição 3.10. Seja $W$ um subespaço de $V$, com $\operatorname{dim} W=k \geq 2$, então são equivalentes:

i. W é tipo-tempo.

ii. $W$ contêm dois vetores tipo-luz linearmente indepenentes.

iii. W contém um vetor tipo-tempo.

Demonstração. (i. $\Rightarrow$ ii.) Como $W$ é tipo-tempo, consideremos uma base ortonormal $\left\{e_{1}, \cdots, e_{k}\right\}$ de $W$, com $e_{1}$ tipo-espaço, e $e_{k}$ tipo-tempo. Então $e_{1}+e_{k}$ e $e_{1}-e_{k}$ são dois vetores tipo-luz linearmente independentes.

(ii. $\Rightarrow$ iii.) Sejam $v, w$ dois vetores tipo-luz linearmente independentes de $W$, então ou $v+w$ ou $v-w$ é tipo-tempo. Isso segue do fato de

$$
g(v \pm w, v \pm w)=g(v, v) \pm 2 g(v, w)+g(w, w)= \pm 2 g(v, w) .
$$

Observe que $g(v, w) \neq 0$, pela Proposição 3.6.

$\left(\right.$ iii. $\Rightarrow i$.) Seja $v$ um vetor tipo-tempo de $W$, logo só precisamos mostrar que $\left.g\right|_{W}$ é não degenerado. Suponha, por contradição, que $\left.g\right|_{W}$ é degenerado. Então existe um vetor $z \neq 0$ tal que $z$ pertence ao radical de $\left.g\right|_{W}$, em particular, $z$ é tipo-luz. Como $v, z$ são linearmente independentes, sabemos da Proposição 3.7 que

- se $v, z$ estão no mesmo cone causal, então $g(v, z)>0$

- se $v, z$ estão em cones causais diferentes, então $g(v, z)<0$.

Ambos os casos contradizem o fato de que $z$ está no radical de $W$. Portanto, $\left.g\right|_{W}$ é não degenerado.

O caso de $W$, quando esse é tipo-espaço, não difere do estudo tradicional de Álgebra Linear, logo não comentaremos. Por outro lado, é possível obter resultados semelhantes ao visto na Proposição 3.10 para um subespaço tipo-luz.

Proposição 3.11. Seja $W$ um subespaço de $V$, então são equivalentes:

i. W é tipo-luz.

ii. W contém um vetor tipo-luz, mas nenhum tipo-tempo.

iii. A intersecção de $W$ com o subconjunto dos vetores nulos (tipo-luz e elemento neutro de $V$ ) forma um subespaço vetorial de dimensão 1.

Demonstração. (i. $\Rightarrow$ ii.) Como $W$ é tipo-luz, então o radical de $g$ em $W$ tem um elemento não nulo, $v \in W$, em particular, $g(v, v)=0$, além disso, $W$ não contém vetor tipo-tempo, caso contrário, $W$ seria tipo-tempo, pela Proposição 3.10 .

(ii. $\Rightarrow$ iii.) Por hipótese, $W$ contém um vetor tipo-luz, $\log$ o $\mathcal{A}$, conjunto dos vetores nulos, é tal que $\operatorname{dim} \mathcal{A} \geq 1$. Contudo, pela Proposição 3.10, $\operatorname{dim} \mathcal{A}=1$, caso contrário existiriam dois vetores tipo-luz linearmente independentes e assim $\mathcal{A}$ conteria um vetor tipo-tempo.

$($ iii. $\Rightarrow$ i.) Note que $W$ não pode ser tipo-tempo, nesse caso $W$ teria dois vetores tipo-luz linearmente independentes (3.10), o que contradiz o fato $\operatorname{de} \operatorname{dim} \mathcal{A}=1$. Além disso, $W$ não 
pode ser tipo-espaço, nesse caso, tomando uma base ortonormal de $W,\left\{e_{1}, \cdots, e_{k}\right\}$, tal que $g_{i i}=1$, para todo $i=1, \cdots, k$, então se $v=\sum_{i=1}^{k} \alpha^{i} e_{i}$ é um vetor tipo-luz de $\mathcal{A}, \operatorname{logo}$

$$
0=g(v, v)=\sum_{i=1}^{k}\left(\alpha^{i}\right)^{2}
$$

$\operatorname{assim} \alpha^{i}=0$, para todo $i=1, \cdots, k, \mathrm{e} \operatorname{dim} \mathcal{A}=0$, também uma contradição. Portanto, como visto na Definição $3.8, W$ deve ser tipo-luz

\subsection{Grupo de Lorentz}

Definição 3.12. O espaço vetorial Lorentziano $\mathbb{L}^{n}=\left(\mathbb{R}^{n},\langle\cdot, \cdot\rangle\right)$, com $n \geq 2$, chamado de espaço-tempo de Lorentz-Minkowski, onde $\langle\cdot, \cdot\rangle$ denota o produto escalar definido por

$$
\left\langle\left(a^{1}, \ldots, a^{n}\right),\left(b^{1}, \ldots, b^{n}\right)\right\rangle=\sum_{i=1}^{n-1} a^{i} b^{i}-a^{n} b^{n},
$$

onde $\left(a^{1}, \ldots, a^{n}\right)$ e $\left(b^{1}, \ldots, b^{n}\right)$ são vetores de $\mathbb{L}^{n}$.

Note que o produto escalar canônico de $\mathbb{L}^{n}$, definido acima, é o mesmo definido no Exemplo 2.25.

Seja $\mathcal{B}_{0}$ a base canônica do $\mathbb{R}^{n}$ e $G L_{n}(\mathbb{R})$ o conjunto das matrizes reais $n \times n$. Denote por

$$
\eta=M_{\mathcal{B}_{0}}(\langle\cdot, \cdot\rangle)=\left(\begin{array}{c|c}
I_{n-1} & 0 \\
\hline 0 & -1
\end{array}\right)
$$

Definição 3.13. Definimos o grupo de transformação de Lorentz como

$$
\operatorname{Iso}\left(\mathbb{L}^{n}\right)=\{f: \mathbb{L} \rightarrow \mathbb{L} \mid f: \text { isometria }\}
$$

e o grupo de Lorentz como

$$
O_{1}(n)=\left\{A \in G L_{n}(\mathbb{R}) \mid A^{t} \eta A=\eta\right\} .
$$

O grupo de Lorentz é o grupo de rotações hiperbólicas nos planos Lorentzianos, como veremos adiante. Ainda, $O_{1}(n)$ é um subgrupo do grupo de Poincaré, que além das rotações contém as translações no espaço-tempo de Lorentz-Minkowski.

Fixe uma base qualquer de $\mathbb{L}^{n}$, podemos construir o grupo de isomorfismos Iso $\left(\mathbb{L}^{n}\right) \rightarrow O_{1}(n)$ que mapeia toda transformação de Lorentz $f$ na matriz $M(f, \mathcal{B})$. Em particular, fixada a base canônica $\mathcal{B}_{0}$, teremos que

$$
\begin{gathered}
\Phi: \operatorname{Iso}\left(\mathbb{L}^{n}\right) \rightarrow O_{1}(n) \\
\Phi(f)=A_{f}=M\left(f, \mathcal{B}_{0}\right)
\end{gathered}
$$

Denote $f_{A}=\Phi^{-1}(A)$. A demonstração de que $\Phi$ é isomorfismo segue de modo análogo ao caso Euclidiano para Iso $\left(\mathbb{R}^{n}\right)$ e $O_{n}$.

Observação 3.14. Como $A^{t} \eta A=\eta$, então segue facilmente que $(\operatorname{det} A)^{2}=1$ e assim $\operatorname{det} f_{A}= \pm 1$, sendo $f_{A}$ o elemento de $\mathrm{Iso}\left(\mathbb{L}^{n}\right)$ tal que $\Phi\left(f_{A}\right)=A$. 
Definição 3.15. Dizemos que uma transformação de Lorentz $f$ é própria se $\operatorname{det}(f)\left(=\operatorname{det} A_{f}\right)=1$. E dizemos que $f$ é imprópria se $\operatorname{det} f=-1$.

O subgrupo das transformações de Lorentz próprias (impróprias) é denotado por $\mathrm{Iso}^{+}\left(\mathbb{L}^{n}\right)$ (respectivamente $\mathrm{Iso}^{-}\left(\mathbb{L}^{n}\right)$ ) e o subgrupo isomorfo em $O_{1}(n)$ denotado por $O_{1}^{+}(n)$ (respectivamente $O_{1}^{-}(n)$ ). Temos que nem $\mathrm{Iso}^{-}\left(\mathbb{L}^{n}\right)$ nem $O_{1}^{-}(n)$ são subgrupos.

Como vimos anteriormente existem dois cones tipo-tempo em $\mathbb{L}^{n}$ conexos. Estabelecer uma orientação temporal terá grande valor adiante e faremos isso usando os cones tipo-tempo e o vetor tipo-tempo na base canônica.

Seja $\left\{e_{1}, \cdots, e_{n}\right\}$ a base canônica de $\mathbb{L}^{n}$, vamos fixar uma orientação temporal usual da seguinte forma:

- Cone causal futuro, $C^{\uparrow}$ : o cone que contém $e_{n}$

- Cone causal passado, $C^{\downarrow}$ : o cone que contém $-e_{n}$.

O estabelecimento de uma orientação temporal permite entender melhor como os elementos de $O_{1}(n)$, em consequência de $\operatorname{Iso}\left(\mathbb{L}^{n}\right)$, operam, como veremos na seguinte observação.

Observação 3.16. Seja $f \in \operatorname{Iso}\left(\mathbb{L}^{n}\right)$ uma transformação de Lorentz, tomando a base canônica $\mathcal{B}=\left\{e_{1}, \ldots, e_{n}\right\}$ temos

$$
A_{f}=\left[\begin{array}{ccc}
a_{11} & \cdots & a_{1 n} \\
\vdots & \ddots & \vdots \\
a_{n 1} & \cdots & a_{n n}
\end{array}\right]
$$

Dessa forma $f\left(e_{n}\right)=a_{1 n} e_{1}+\cdots+a_{n n} e_{n}$. Observe que

$$
-1=\left\langle e_{n}, e_{n}\right\rangle=\left\langle f\left(e_{n}\right), f\left(e_{n}\right)\right\rangle=a_{1 n}^{2}+\cdots+a_{(n-1) n}^{2}-a_{n n}^{2},
$$

então $a_{n n}^{2}-1 \geq 0$ e $\left|a_{n n}\right| \geq 1$. Como $\left\langle e_{n}, f\left(e_{n}\right)\right\rangle=-a_{n n}$, temos que são equivalentes:

i. $a_{n n} \geq 1$

ii. $f\left(e_{n}\right) \in C^{\uparrow}$

iii. $f\left(C^{\uparrow}\right)=C^{\uparrow}$

iv. $f\left(C^{\downarrow}\right)=C^{\downarrow}$

Demonstração. (i. $\Rightarrow$ ii.) Por hipótese $a_{n n} \geq 1$, logo $\left\langle f\left(e_{n}\right), e_{n}\right\rangle=-a_{n n}<0$ e, pela Proposição $3.2, e_{n}$ e $f\left(e_{n}\right)$ estão no mesmo cone tipo-tempo. Portanto $f\left(e_{n}\right) \in C^{\uparrow}$.

(ii. $\Rightarrow$ iii.) Óbvio, pois como $f\left(e_{n}\right) \in C^{\uparrow}$, então temos, pela Proposição 3.2 que

$$
v \in C^{\uparrow} \Leftrightarrow\left\langle v, e_{n}\right\rangle<0 \Leftrightarrow\left\langle f(v), f\left(e_{n}\right)\right\rangle<0 \Leftrightarrow f(v) \in C^{\uparrow} .
$$

$($ iii. $\Rightarrow$ iv. $)$ Assim como no item anterior, pela Proposição 3.2 e como $f\left(C^{\uparrow}\right)=C^{\uparrow}$

$$
v \in C^{\downarrow} \Leftrightarrow\left\langle v, e_{n}\right\rangle>0 \Leftrightarrow\left\langle f(v), f\left(e_{n}\right)\right\rangle>0 \Leftrightarrow f(v) \in C^{\downarrow} .
$$

$\left(i v . \Rightarrow i\right.$.) Por hipótese $f\left(C^{\downarrow}\right)=C^{\downarrow}$, então $\left\langle f\left(-e_{n}\right), e_{n}\right\rangle>0$. Assim, $\left.-\left\langle f\left(e_{n}\right), e_{n}\right\rangle=a_{n n}\right\rangle$ 0. Portanto, como $\left|a_{n n}\right| \geq 1, a_{n n} \geq 1$. 
De modo análogo podemos obter

$$
a_{n n} \leq-1 \Leftrightarrow f\left(e_{n}\right) \in C^{\downarrow} \Leftrightarrow f\left(C^{\uparrow}\right)=C^{\downarrow} \Leftrightarrow f\left(C^{\downarrow}\right)=C^{\uparrow} .
$$

Definição 3.17. Seja $f \in \operatorname{Iso}\left(\mathbb{L}^{n}\right)$, dizemos que $f$ é ortocronos se $f\left(C^{\uparrow}\right)=C^{\uparrow}$. Iremos denotar por $\operatorname{Iso}^{\uparrow}\left(\mathbb{L}^{n}\right)$ o subgrupo das transformações ortocronos e $O_{1}^{\uparrow}(n)$ o subgrupo correspondente em $O_{1}(n)$. Analogamente, denotaremos o subconjunto das transformações não-ortocronos por $\operatorname{Iso}^{\downarrow}\left(\mathbb{L}^{n}\right)$ e, respectivamente, $O_{1}^{\downarrow}(n)$.

Portanto, pela Observação 3.16, $f \in \operatorname{Iso}^{\uparrow}\left(\mathbb{L}^{n}\right)$ se, e somente se, $a_{n n} \geq 1$, sendo $a_{n n} \mathrm{o}$ elemento $(n, n)$ de $M_{\mathcal{B}_{0}}(f)$ com $\mathcal{B}_{0}$ sendo a base canônica de $\mathbb{L}^{n}$.

De agora em diante combinaremos os conceitos definidos em 3.15 e 3.17, denotando as combinações da seguinte forma:

- $O_{1}^{+\uparrow}(n)$ : transformações ortocronos próprias;

- $O_{1}^{-\uparrow}(n)$ : transformações ortocronos impróprias;

- $O_{1}^{+\downarrow}(n)$ : transformações não-ortocronos próprias;

- $O_{1}^{-\downarrow}(n)$ : transformações não-ortocronos impróprias.

Exemplo 3.18. Pelas definições 3.15 e 3.17 temos os seguintes exemplos:

$$
\begin{aligned}
& {\left[\begin{array}{c|cc}
I_{n-2} & 0 & 0 \\
\hline 0 & 1 & 0 \\
0 & 0 & 1
\end{array}\right] \in S O_{1}^{\uparrow}(n) \quad\left[\begin{array}{c|cc}
I_{n-2} & 0 & 0 \\
\hline 0 & -1 & 0 \\
0 & 0 & -1
\end{array}\right] \in O_{1}^{+\downarrow}} \\
& {\left[\begin{array}{c|cc}
I_{n-2} & 0 & 0 \\
\hline 0 & -1 & 0 \\
0 & 0 & 1
\end{array}\right] \in O_{1}^{-\uparrow}(n) \quad\left[\begin{array}{c|cc}
I_{n-2} & 0 & 0 \\
\hline 0 & 1 & 0 \\
0 & 0 & -1
\end{array}\right] \in O_{1}^{-\downarrow}(n)}
\end{aligned}
$$

O exemplo acima mostra que o grupo $O_{1}(n)$ têm pelo menos 4 componentes conexas. De fato, ele têm 4 e deixamos [VC10] e [O'N83] como referências para demonstração e resultados.

O caso de $O_{1}^{+\uparrow}(n)$ é especial por ser o único subgrupo de $O_{1}(n)$, logo será denotado por $S O_{1}^{\uparrow}(n)$, chamado de grupo de Lorentz restrito.

Proposição 3.19. Seja $f \in \operatorname{Iso}\left(\mathbb{L}^{n}\right)$, então são equivalentes:

i. $f \in \operatorname{Iso}^{\uparrow}\left(\mathbb{L}^{n}\right)$,

ii. existe um vetor causal $v \in \mathbb{L}^{n}$ tal que $\langle v, f(v)\rangle<0$,

iii. para todo vetor tipo-tempo $v \in \mathbb{L}^{n}$ vale que $\langle v, f(v)\rangle<0$,

iv. para toda base ortonormal, o elemento $(n, n)$ da matriz de $f$ é maior que zero (e, de fato, maior ou igual a 1).

Demonstração. (i. $\Rightarrow$ ii.) Suponha que $f \in \operatorname{Iso}^{\uparrow}\left(\mathbb{L}^{n}\right)$, logo o elemento $(n, n)$ da matriz de $f$ na base canônica é maior que zero, portanto, como visto na Observação 3.16, $\left\langle e_{n}, f\left(e_{n}\right)\right\rangle<0$.

(ii. $\Rightarrow$ iii.) Seja, por hipótese, $w \in \mathbb{L}^{n}$ um vetor causal tal que

$$
\langle w, f(w)\rangle<0
$$


logo estão no mesmo cone pela Proposição 3.7. Considere $v \in \mathbb{L}^{n}$ um vetor tipo-tempo. Se $\{v, w\}$ é linearmente dependente, então $v=\alpha w \mathrm{e}$

$$
\langle v, f(v)\rangle=\langle\alpha w, \alpha f(w)\rangle=\alpha^{2}\langle w, f(w)\rangle<0,
$$

portanto vale o resultado. Suponha então que $\{v, w\}$ é linearmente independente. Dessa forma $\langle v, w\rangle \neq 0$, portanto teremos que

- se $\langle v, w\rangle<0$, então $\langle f(v), f(w)\rangle<0$; assim $v, w$ estão no mesmo cone e $f(v), f(w)$ também. Com isso, pela equação 3.3, $v, f(v)$ estão no mesmo cone e vale o resultado.

- se $\langle v, w\rangle>0$, então $\langle f(v), f(w)\rangle>0$; assim $v, w$ estão em cones diferentes e $f(v), f(w)$ também estão. Com isso, pela equação $3.3, v, f(v)$ estão no mesmo cone e vale o resultado.

iii. $\Rightarrow i v$. Seja $\left\{u_{1}, \ldots, u_{n-1}, v\right\}$ uma base ortonormal de $\mathbb{L}^{n}$, sendo $v$ tipo-tempo. Suponha que

$$
f(v)=\alpha_{1} u_{1}+\ldots+\alpha_{n-1} u_{n-1}+\alpha^{n} v,
$$

note que os elementos $\alpha_{i}$ compõe a $n$-ésima coluna da matriz de $f$. Além disso,

$$
\langle v, f(v)\rangle=-\alpha_{n}
$$

e, por hipótese, $\langle v, f(v)\rangle<0$, logo $\alpha_{n}>0$ e portanto vale o resultado.

$i v . \Rightarrow i$. Note que a Observação 3.16 vale para qualquer base ortonormal de $\mathbb{L}^{n}$, não somente a base canônica.

\subsubsection{O grupo de Lorentz em dimensão 2}

No que segue, iremos determinar o grupo de isometrias $O_{1}(2)$, isto é, queremos determinar todas as matrizes $2 \times 2$ tais que

$$
A^{t} \cdot\left[\begin{array}{cc}
1 & 0 \\
0 & -1
\end{array}\right] \cdot A=\left[\begin{array}{cc}
1 & 0 \\
0 & -1
\end{array}\right]
$$

Seja $\mathcal{B}_{0}=\left\{e_{1}, e_{2}\right\}$ a base canônica de $\mathbb{L}^{2}=\left(\mathbb{R}^{2},\langle\cdot, \cdot\rangle\right)$ e consideremos a base $\mathcal{B}=\{u, v\}$, onde

$$
u=\frac{1}{\sqrt{2}}\left(e_{1}+e_{2}\right) \quad \text { e } \quad v=\frac{1}{\sqrt{2}}\left(-e_{1}+e_{2}\right)
$$

Note que $u$ e $v$ são vetores do tipo-luz, de fato

$$
\begin{gathered}
\langle u, u\rangle=\left\langle\frac{1}{\sqrt{2}}\left(e_{1}+e_{2}\right), \frac{1}{\sqrt{2}}\left(e_{1}+e_{2}\right)\right\rangle=\frac{1}{2}\left(\left\langle e_{1}, e_{1}\right\rangle+\left\langle e_{2}, e_{2}\right\rangle\right)=0 \\
\langle v, v\rangle=\left\langle\frac{1}{\sqrt{2}}\left(-e_{1}+e_{2}\right), \frac{1}{\sqrt{2}}\left(-e_{1}+e_{2}\right)\right\rangle=\frac{1}{2}\left(\left\langle e_{1}, e_{1}\right\rangle+\left\langle e_{2}, e_{2}\right\rangle\right)=0 .
\end{gathered}
$$

Além disso, $u$ e $v$ estão no mesmo cone causal, pois

$$
\langle u, v\rangle=\frac{1}{2}\left\langle e_{1}+e_{2},-e_{1}+e_{2}\right\rangle=\frac{1}{2}\left(-\left\langle e_{1}, e_{1}\right\rangle+\left\langle e_{2}, e_{2}\right\rangle\right)=-1 .
$$


Tomando tal base temos então que

$$
M_{\mathcal{B}}(\langle\cdot, \cdot\rangle)=\left[\begin{array}{cc}
0 & -1 \\
-1 & 0
\end{array}\right]
$$

E ainda fica definida a matriz de mudança de base $\mathcal{B}_{0} \leftarrow \mathcal{B}, P$,

$$
P=\frac{1}{\sqrt{2}}\left[\begin{array}{cc}
1 & 1 \\
1 & -1
\end{array}\right] \quad \text { e } \quad P^{-1}=\frac{1}{\sqrt{2}}\left[\begin{array}{cc}
1 & 1 \\
-1 & 1
\end{array}\right]
$$

Para explicitar os elementos de $O_{1}(2)$ vamos obter a matriz de cada $f \in \operatorname{Iso}\left(\mathbb{L}^{2}\right)$ na base $\mathcal{B}$ e então na base $\mathcal{B}_{0}$ usando a mudança $M\left(f, \mathcal{B}_{0}\right)=P \cdot M(f, \mathcal{B}) \cdot P^{-1}$.

Logo, escrevendo $f(u)=a u+b v$ e $f(v)=\bar{a} u+\bar{b} v$, com $a, b, \bar{a}, \bar{b} \in \mathbb{R}$, teremos, como $f$ é isometria,

$$
\begin{aligned}
& 0=\langle u, u\rangle=\langle f(u), f(u)\rangle=-2 a b, \\
& 0=\langle v, v\rangle=\langle f(v), f(v)\rangle=-2 \bar{a} \bar{b} .
\end{aligned}
$$

Agora temos dois caso exclusivos: Caso I: $b=0$ ou Caso II: $a=0$.

- Caso I: nesse caso $f(u)=\lambda u(\lambda \neq 0)$. Como $f \in \operatorname{Iso}\left(\mathbb{L}^{2}\right)$ então

$$
-1=\langle u, v\rangle=\langle f(u), f(v)\rangle=\langle\lambda u, \bar{a} u+\bar{b} v\rangle=-\lambda \bar{b},
$$

nesse caso temos que $\bar{b}=\frac{1}{\lambda}$ e, necessariamente, pela equação $3.5, \bar{a}=0$. Dessa forma, $f(v)=\frac{1}{\lambda} v$ e então

$$
M(f, B)=\left[\begin{array}{ll}
\lambda & 0 \\
0 & \frac{1}{\lambda}
\end{array}\right] .
$$

Portanto $f \in \operatorname{Iso}^{+}\left(\mathbb{L}^{2}\right)$ (pois $\operatorname{det} f=1$ ) e como $f(u)=\lambda u$ teremos que $\langle v, f(u)\rangle=\langle v, \lambda u\rangle=-\lambda$, portanto $f(u)$ está no mesmo cone de $u$ e $v$, ou seja, $f \in \operatorname{Iso}^{\uparrow}\left(\mathbb{L}^{2}\right)$, se, e somente se, $\lambda>0$.

- Caso II: nesse caso $f(u)=\lambda v$. Como no caso anterior, usando a equação 3.4, teremos que $f(v)=\frac{1}{\lambda} u$ e então

$$
M(f, B)=\left[\begin{array}{cc}
0 & \frac{1}{\lambda} \\
\lambda & 0
\end{array}\right] .
$$

Assim, $f \in \operatorname{Iso}^{-}\left(\mathbb{L}^{2}\right)(\operatorname{det} f=-1)$ e da mesma forma, como $f(u)=\lambda v$ teremos $\langle u, f(u)\rangle=\langle u, \lambda v\rangle=-\lambda$, portanto $f(u)$ está no mesmo cone de $u$ e $v$, ou seja, $f \in \operatorname{Iso}^{\uparrow}\left(\mathbb{L}^{2}\right)$, se, e somente se, $\lambda>0$.

Podemos agora determinar como são os elementos de $O_{1}(2)$. Logo, se $f \in \operatorname{Iso}\left(\mathbb{L}^{2}\right)$, então

$$
A_{f} \in O_{1}^{+\uparrow}(2) \Leftrightarrow M(f, \mathcal{B})=\left[\begin{array}{cc}
\lambda & 0 \\
0 & \frac{1}{\lambda}
\end{array}\right],
$$


para $\lambda>0$. Como vimos acima, $M\left(f, \mathcal{B}_{0}\right)=P \cdot M(f, \mathcal{B}) \cdot P^{-1}$, assim

$$
M\left(f, \mathcal{B}_{0}\right)=\frac{1}{2}\left[\begin{array}{cc}
\lambda+\frac{1}{\lambda} & \lambda-\frac{1}{\lambda} \\
\lambda-\frac{1}{\lambda} & \lambda+\frac{1}{\lambda}
\end{array}\right] .
$$

Tomando $\theta=\ln \lambda \in \mathbb{R}$ teremos então

$$
\lambda+\frac{1}{\lambda}=e^{\theta}+e^{-\theta} \quad \text { e } \quad \lambda-\frac{1}{\lambda}=e^{\theta}-e^{-\theta},
$$

portanto

$$
M\left(f, \mathcal{B}_{0}\right)=\left[\begin{array}{cc}
\cosh \theta & \sinh \theta \\
\sinh \theta & \cosh \theta
\end{array}\right]
$$

Da mesma forma, consideremos agora $f \in O_{1}^{+\downarrow}(2)$. Então, $\operatorname{det} f=1$, logo

$$
M(f, \mathcal{B})=\left[\begin{array}{ll}
\lambda & 0 \\
0 & \frac{1}{\lambda}
\end{array}\right],
$$

para $\lambda<0$. Assim,

$$
M\left(f, \mathcal{B}_{0}\right)=\frac{1}{2}\left[\begin{array}{cc}
\lambda+\frac{1}{\lambda} & \lambda-\frac{1}{\lambda} \\
\lambda-\frac{1}{\lambda} & \lambda+\frac{1}{\lambda}
\end{array}\right]
$$

e tomando $\theta=\ln -\lambda$ teremos

$$
\lambda+\frac{1}{\lambda}=-\left(e^{\theta}+e^{-\theta}\right) \quad \text { e } \quad \lambda-\frac{1}{\lambda}=-\left(e^{\theta}-e^{-\theta}\right),
$$

portanto

$$
M\left(f, \mathcal{B}_{0}\right)=\left[\begin{array}{ll}
-\cosh \theta & -\sinh \theta \\
-\sinh \theta & -\cosh \theta
\end{array}\right] .
$$

Tomemos agora $f \in O_{1}^{-\uparrow}, \operatorname{logo} \operatorname{det} f=-1$. Então

$$
M(f, \mathcal{B})=\left[\begin{array}{cc}
0 & \frac{1}{\lambda} \\
\lambda & 0
\end{array}\right],
$$

para $\lambda>0$. Assim,

$$
M\left(f, \mathcal{B}_{0}\right)=\frac{1}{2}\left[\begin{array}{cc}
-\lambda-\frac{1}{\lambda} & -\lambda+\frac{1}{\lambda} \\
\lambda-\frac{1}{\lambda} & \lambda+\frac{1}{\lambda}
\end{array}\right]
$$

e tomando $\theta=\ln \lambda$ teremos

$$
\lambda+\frac{1}{\lambda}=e^{\theta}+e^{-\theta} \quad \text { e } \quad \lambda-\frac{1}{\lambda}=e^{\theta}-e^{-\theta},
$$


portanto

$$
M\left(f, \mathcal{B}_{0}\right)=\left[\begin{array}{cc}
-\cosh \theta & -\sinh \theta \\
\sinh \theta & \cosh \theta
\end{array}\right]
$$

Semelhante ao que fizemos com $O_{1}^{+\downarrow}$, se $f \in O_{1}^{-\downarrow}$, então

$$
M\left(f, \mathcal{B}_{0}\right)=\left[\begin{array}{cc}
\cosh \theta & \sinh \theta \\
-\sinh \theta & -\cosh \theta
\end{array}\right]
$$

Em resumo, temos que os elementos de $O_{1}(2)$, separados nas suas 4 componentes, podem ser dos seguintes tipos:

$$
\begin{array}{cc}
S O_{1}^{\uparrow}(2):\left[\begin{array}{cc}
\cosh \theta & \sinh \theta \\
\sinh \theta & \cosh \theta
\end{array}\right] & O_{1}^{+\downarrow}(2):\left[\begin{array}{cc}
-\cosh \theta & -\sinh \theta \\
-\sinh \theta & -\cosh \theta
\end{array}\right] \\
O_{1}^{-\uparrow}(2):\left[\begin{array}{cc}
-\cosh \theta & -\sinh \theta \\
\sinh \theta & \cosh \theta
\end{array}\right] & O_{1}^{-\downarrow}(2):\left[\begin{array}{cc}
\cosh \theta & \sinh \theta \\
-\sinh \theta & -\cosh \theta
\end{array}\right]
\end{array}
$$

Observação 3.20. $\quad i$. Observe que se $\theta=0$ então as matrizes acima ficam reduzidas às seguintes formas

$$
\begin{array}{cc}
S O_{1}^{\uparrow}(2):\left[\begin{array}{ll}
1 & 0 \\
0 & 1
\end{array}\right] & O_{1}^{+\downarrow}(2):\left[\begin{array}{cc}
-1 & 0 \\
0 & -1
\end{array}\right] \\
O_{1}^{-\uparrow}(2):\left[\begin{array}{cc}
-1 & 0 \\
0 & 1
\end{array}\right] & O_{1}^{-\downarrow}(2):\left[\begin{array}{cc}
1 & 0 \\
0 & -1
\end{array}\right]
\end{array}
$$

O que é compatível com o que vimos no Exemplo 3.18.

ii. É possível ver pela própria construção feita no Caso I que para qualquer $\theta \in \mathbb{R}$ as isometrias de $S O_{1}^{\uparrow}$ e $O_{1}^{+\downarrow}$ são diagonalizáveis com mesma base de autovetores do tipoluz e, a menos de sinal, os mesmos autovalores.

iii. Consideremos então um elemento de $O_{1}^{-\uparrow}$, com $\theta \neq 0$, e calculemos seus autovetores. Seja

$$
(A-\lambda \mathrm{Id})=\left[\begin{array}{cc}
-\cosh \theta-\lambda & -\sinh \theta \\
\sinh \theta & \cosh \theta-\lambda
\end{array}\right]
$$

Então

$$
\operatorname{det}(A-\lambda \mathrm{Id})=-(\cosh \theta+\lambda) \cdot(\cosh \theta-\lambda)+\sinh ^{2} \theta
$$

e fazendo $\operatorname{det}(A-\lambda \mathrm{Id})=0$ teremos que o polinômio característico de $A$ é

$$
c_{A}(\lambda)=\lambda^{2}-1
$$

pois $\cosh ^{2} \theta-\sinh ^{2} \theta=1$. Logo, os autovalores de $A$ são $+1 e-1$.

Para $\lambda=1$ temos o seguinte sistema

$$
\left\{\begin{array}{c}
-(\cosh \theta+1) x-\sinh \theta y=0 \\
\sinh \theta x+(\cosh \theta-1) y=0
\end{array}\right.
$$

donde obtemos o autovetor

$$
\left(1,-\frac{(\cosh \theta+1)}{\sinh \theta}\right) .
$$


Para $\lambda=-1$ temos o seguinte sistema

$$
\left\{\begin{array}{c}
(-\cosh \theta+1) x-\sinh \theta y=0 \\
\sinh \theta x+(\cosh \theta+1) y=0
\end{array}\right.
$$

donde obtemos o autovetor

$$
\left(1, \frac{-\cosh \theta+1}{\sinh \theta}\right)
$$

E é possivel ver que tais autovetores são linearmente independetes entre si. Portanto, os elementos de $O_{1}^{-\uparrow}$ são diagonalizáveis e, por consequência, os elementos de $O_{1}^{-\downarrow}$ também o são.

\subsubsection{Grupos de Lorentz em dimensões maiores}

Sabendo explicitamente quais são os elementos do grupo de Lorentz em dimensão 2, podemos obter algumas propriedades do grupo de Lorentz para dimensão arbitrária $n, O_{1}(n)$.

Proposição 3.21. Seja $A \in O_{1}(n)$, tomando a métrica canônica de $\mathbb{L}^{n}$ $\langle\cdot, \cdot\rangle=-d x_{n}^{2}+\sum d x_{i}^{2}$, então:

i. Os autovetores não tipo-luz de A, se existem, estão associados aos autovalores 1 ou -1 .

ii. O produto de autovalores associados a dois autovetores tipo-luz linearmente independentes é igual a 1.

iii. Se $V$ é um autoespaço de $A$ associado a um autovetor não tipo-luz, então qualquer outro autoespaço é ortogonal a $V$.

iv. Se $V$ é um subespaço A-invariante, então $V^{\perp}$ também é A-invariante.

Demonstração. $\quad i$. Seja $v$ um autovetor não tipo-luz de $A, \operatorname{logo} A . v=\lambda v$. Então, como $A$ é isometria, temos

$$
\langle v, v\rangle=\langle A . v, A . v\rangle=\lambda^{2}\langle v, v\rangle
$$

e então, como $\langle v, v\rangle \neq 0, \lambda= \pm 1$.

ii. Sejam $v$ e $w$ autovetores tipo-luz de $A$ linenarmente independentes, logo $A . v=\alpha v$ e $A . v=\beta v$. Então

$$
\langle v, w\rangle=\langle A . v, A . w\rangle=\alpha \beta\langle v, w\rangle
$$

e, pela Proposição 3.6, $\langle v, w\rangle \neq 0$. Portanto, $\alpha \beta=1$.

iii. Seja $V$ o autoespaço associado ao autovetor não tipo-luz de $A, v$. Pelo item i., temos que $A . v=\varepsilon v$, onde $\varepsilon= \pm 1$. Seja $w$ um autovetor de $A$ associado ao autovalor $\lambda$ tal que $\lambda \neq \varepsilon$. Então

$$
\langle v, w\rangle=\langle A . v, A . w\rangle=\varepsilon \lambda\langle v, w\rangle .
$$

Nessa caso, se $\langle v, w\rangle \neq 0$, então $\varepsilon \lambda=1$ o que implica $\lambda=\varepsilon$, contradizendo a hipótese feita para $\lambda$. Portanto, $\langle v, w\rangle=0$ e os autoespaços gerados são ortogonais.

iv. Por hipótese, $V$ é $A$-invariante, $\log o A(V) \subset V$, porém, como $A$ é isometria, então $A(V)=V$. Dessa forma, temos que $A^{-1}(V)=V$. Seja $w \in V^{\perp}$, então, para todo $v \in V$

$$
\langle A . w, v\rangle=\left\langle w, A^{-1} \cdot v\right\rangle=0
$$


ou seja, A.w $\in V^{\perp}$. Portanto, $A\left(V^{\perp}\right) \subset V$ e $V^{\perp}$ é $A$-invariante.

A Definição 3.8 propõe uma abordagem através de subespaços para estudar as propriedades de um espaço Lorentziano, separando-os em caráteres causais, como feito com vetores. Fizemos isso nas proposições 3.10 e 3.11. Podemos fazer algo semelhante ao estudar o grupo de Lorentz, tomando seus elementos restritos aos subespaços tipo-tempo e tipo-luz.

Definição 3.22. Seja $\pi$ um subconjunto de $\mathbb{L}^{n}$, com $n \geq 2$. Dizemos que $\pi$ é um plano se $\pi$ for um subespaço vetorial de $\mathbb{L}^{n}$ e $\operatorname{dim} \pi=2$.

Proposição 3.23. Considere $A \in O_{1}(n)$. Seja $\pi \subset \mathbb{L}^{n}$ um plano A-invariante tipo-tempo. Então somente uma das seguintes possibilidades ocorre:

i. $\left.A\right|_{\pi} e^{\prime} \pm \operatorname{Id}_{n}$.

ii. $\left.A\right|_{\pi}$ admite dois autovetores tipo-luz linearmente independentes com autovalores $\lambda e$ $\frac{1}{\lambda}, \operatorname{com} \lambda \neq \pm 1$.

iii. $\left.A\right|_{\pi}$ admite dois autovetores não tipo-luz linearmente independentes sendo um deles associado a 1 e o outro associado a -1 .

Demonstração. Como $\operatorname{dim} \pi=2$, então $\left.A\right|_{\pi} \in O_{1}(2)$. Dessa forma, a prova desse resultado segue diretamente da Observação 3.20.

Se tomarmos $\theta=0$, teremos que $\left.A\right|_{\pi}$ é igual a

$$
\left[\begin{array}{ll}
1 & 0 \\
0 & 1
\end{array}\right] \quad \text { ou } \quad\left[\begin{array}{cc}
-1 & 0 \\
0 & -1
\end{array}\right]
$$

dependendo se $\left.A\right|_{\pi} \in S O_{1}^{\uparrow}(2)$ ou $\left.A\right|_{\pi} \in O_{1}^{+\downarrow}(2)$, respectivamente, o que contempla a possibilidade $i$.

Ainda $\operatorname{com} \theta=0$, se $\left.A\right|_{\pi} \in O_{1}^{-\uparrow}(2)$ ou $\left.A\right|_{\pi} \in O_{1}^{-\downarrow}(2)$, então temos

$$
\left[\begin{array}{cc}
-1 & 0 \\
0 & 1
\end{array}\right] \quad \text { ou } \quad\left[\begin{array}{cc}
1 & 0 \\
0 & -1
\end{array}\right]
$$

respectivamente. Ambas as matrizes estão escritas nas bases canônicas com um autovetor tipo-espaço e um autovetor tipo-tempo; e autovalores 1 e - 1 . O que contempla a possibilidade iii.

Tomamos agora $\theta \neq 0$. Para os casos $\left.A\right|_{\pi} \in S O_{1}^{\uparrow}$ e $\left.A\right|_{\pi} \in O_{1}^{+\downarrow}$, já vimos que ambas são diagonalizáveis com autovetores tipo-luz e autovalores associados $\lambda$ e $\frac{1}{\lambda}$, a menos de sinal entre os casos. Além disso, como estamos supondo $\theta \neq 0$, teremos:

- $\lambda \neq 1$, para o caso $\left.A\right|_{\pi} \in S O_{1}^{\uparrow}$, pois fizemos $\theta=\ln \lambda$;

- $\lambda \neq-1$, para o caso $\left.A\right|_{\pi} \in O_{1}^{+\downarrow}$, pois fizemos $\theta=\ln -\lambda$.

Isso contempla a possibilidade $i$.

Para os casos $\left.A\right|_{\pi} \in O_{1}^{-\uparrow}(2)$ ou $\left.A\right|_{\pi} \in O_{1}^{-\downarrow}(2)$ vimos que em ambos os autovalores são 1 e -1 , para os mesmos autovetores, a menos de sinal. Resta provar apenas que não são tipo-luz. 
- Para o autovetor $u=\left(1,-\frac{(\cosh \theta+1)}{\sinh \theta}\right)$ temos

$$
\langle u, u\rangle=1-\frac{(\cosh \theta+1)^{2}}{\sinh ^{2} \theta}=\frac{\sinh ^{2} \theta}{\sinh ^{2} \theta}-\frac{\cosh ^{2} \theta+2 \cosh \theta+1}{\sinh ^{2} \theta}=\frac{-2(\cosh \theta+1)}{\sinh ^{2} \theta},
$$

$\log 0\langle u, u\rangle=0 \Leftrightarrow \cosh \theta=-1$, mas $\cosh \theta>0$, para qualquer $\theta \in \mathbb{R}$. Portanto, $u$ é tipo-tempo.

- Para o autovetor $v=\left(1, \frac{-\cosh \theta+1}{\sinh \theta}\right)$ temos

$$
\langle v, v\rangle=1-\frac{(-\cosh \theta+1)^{2}}{\sinh ^{2} \theta}=\frac{\sinh ^{2} \theta}{\sinh ^{2} \theta}-\frac{\cosh ^{2} \theta-2 \cosh \theta+1}{\sinh ^{2} \theta}=\frac{2(\cosh \theta-1)}{\sinh ^{2} \theta},
$$

$\operatorname{logo}\langle v, v\rangle=0 \Leftrightarrow \cosh \theta=1$, mas $\cosh \theta=1 \Leftrightarrow \theta=0$. Portanto, $v$ é tipo-espaço.

Isso contempla a possibilidade $i$ ii.

Proposição 3.24. Considere $A \in O_{1}(n)$. Seja $\pi \subset \mathbb{L}^{n}$ um plano A-invariante tipo-luz. Então $\left.A\right|_{\pi}$ admite um autovetor tipo-luz $v$, com $A . v=\lambda v$. Se $\lambda \neq \pm 1$, então $\left.A\right|_{\pi}$ admite também um autovetor tipo-epsço.

Demonstração. Pela Proposição 3.11, os vetores nulos (tipo-luz e vetor zero) de $\pi$ formam um subespaço de dimensão 1 . Seja $v \in \pi$, um vetor tipo-luz, considere $A . v$ e, como $A$ é uma isometria, temos que $A . v$ também é tipo-luz, então $A . v=\lambda v$, para algum $\lambda \in \mathbb{R}$. Seja $w \in \pi$ um vetor não tipo-luz, como $\pi$ é um subespaço tipo-luz, então obrigatoriamente $w$ é tipo-espaço, já que não há vetores tipo-tempo em $\pi$ (Proposição 3.11, item ii.). Dessa forma, $A . w=\alpha w+\beta v$, para algum $\alpha, \beta \in \mathbb{R}$. Então, como $A$ é isometria,

$$
\langle w, w\rangle=\langle A . w, A . w\rangle=\langle\alpha w+\beta v, \alpha w+\beta v\rangle=\alpha^{2}\langle w, w\rangle+2 \alpha \beta\langle w, v\rangle+\beta^{2}\langle v, v\rangle=\alpha^{2}\langle w, w\rangle .
$$

$\mathrm{E}$ assim, $\alpha= \pm 1$. Note que $\{w, v\}$ é base de $\pi$ e como $A . w= \pm w+\beta v$ e $A \cdot v=\lambda v$ temos

$$
\left.A\right|_{\pi}=\left[\begin{array}{cc} 
\pm 1 & 0 \\
\beta & \lambda
\end{array}\right]
$$

De onde tiramos que o polinômio característico é

$$
c_{\left.A\right|_{\pi}}(\delta)=(\delta \mp 1)(\delta-\lambda) .
$$

Portanto, se $\lambda \neq \pm 1$ o polinômio característico tem duas raízes distintas e $\left.A\right|_{\pi}$ é diagonalizável com um autovetor tipo-espaço.

Observação 3.25. As proposições 3.23 e 3.24 mostram que se $\pi$ é um plano não tipoespaço, então $\left.A\right|_{\pi}$ sempre admite um autovetor causal $e$ ambas as raízes do polinômio característico são reais.

Lema 3.26. Seja $A \in O_{1}(n)$. Se o polinômio característico de A admite uma raiz complexa não real $\alpha$, então existe um plano A-invariante $\pi$ do tipo-espaço tal que as raízes de $\left.A\right|_{\pi}$ são $\alpha$ e seu conjugado, $\bar{\alpha}$.

Demonstração. Seja $z \in \mathbb{C}$ um autovetor complexo de $A$ associado ao autovalor complexo $\alpha, \log A . z=\alpha z$. Dessa forma, temos que $A \cdot \bar{z}=\bar{\alpha} \bar{z}$, pois $A$ é uma matriz com elementos 
reais. Sabemos que $\{z, \bar{z}\}$ é um conjunto linearmente independente, então geram o plano complexo $\operatorname{span}_{\mathbb{C}}(z, \bar{z})$. Denominamos então

$$
x=\operatorname{Re}(z)=\frac{z+\bar{z}}{2} \quad \text { e } \quad y=\operatorname{Im}(z)=\frac{z-\bar{z}}{2 i} .
$$

E temos que $\{x, y\}$, com $x, y \in \mathbb{R}^{n}$, é um conjunto linearmente independente, não só tomando escalares reais, mas também escalares complexos. Logo, podemos considerar o plano

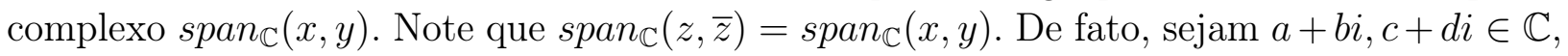
então

$$
\begin{aligned}
(a+b i) z+(c+d i) \bar{z}=(a+b i) & (x+y i)+(c+d i)(x-y i) \\
= & a x+a u i+b x i-b y+c x-c y i+d x i+d y \\
= & {[(a+c)+(b+d) i] x+[(d-b)+(a-c) i] y . }
\end{aligned}
$$

Defina $\pi=\operatorname{span}_{\mathbb{R}}(x, y)$. Sejam $a, b \in \mathbb{R}$ e $\alpha=\gamma i$, então

$$
\begin{array}{r}
a A . x+b A \cdot y=a\left(\frac{A \cdot z+A \cdot \bar{z}}{2}\right)+b\left(\frac{A \cdot z-A \cdot \bar{z}}{2 i}\right)=\left(\frac{a-b i}{2}\right) A \cdot z+\left(\frac{a+b i}{2}\right) A \cdot \bar{z} \\
=\left(\frac{a-b i}{2}\right) \alpha z+\left(\frac{a+b i}{2}\right) \bar{\alpha} \bar{z}=\frac{1}{2}[(a \gamma i+b \gamma)(x+y i)+(-a \gamma i+b \gamma)(x-y i)] \\
\quad=\frac{1}{2}[a \gamma x i-a \gamma y+b \gamma x+b \gamma y i-a \gamma x i-a \gamma y+b \gamma x-b \gamma y i]=(b \gamma) x-(a \gamma) y .
\end{array}
$$

Então $\pi$ é $A$-invariante. Como $\mathcal{S}=\operatorname{span}_{\mathbb{C}}(x, y)=\operatorname{span}_{\mathbb{C}}(z, \bar{z})$, então os polinômios característicos são iguais, ou seja,

$$
c_{\left.A\right|_{\mathcal{S}}}(T)=(T-\alpha)(T-\bar{\alpha}),
$$

portanto $c_{A \mid \pi}$ é irredutível em $\mathbb{R}$ e tem raízes $\alpha$ e $\bar{\alpha}$. Por fim, pela Observação $3.25, \pi$ é um plano tipo-espaço, pois, caso contrário, o polinômio característico de $\left.A\right|_{\pi}$ teria ambas as raízes reais.

Lema 3.27. Seja $A \in O_{1}(n)$, com $n \geq 2$. Se $A$ admite um autovetor, então $A$ admite um autovetor causal.

Demonstração. Provaremos o resultado fazendo indução sobre $n$. Suponha $n=2$, nesse caso, estamos nas hipóteses da Proposição 3.23 e segue o resultado. Suponha, por hipótese de indução, que o resultado vale para todo $k<n$ e vamos provar para $k=n$. Seja $\lambda \neq 0$ uma raiz do polinômio característico, logo:

- se $\lambda \in \mathbb{R}$, então $c_{A}(x)=(x-\lambda) p(x), \operatorname{deg} p(x)=n-1$, e $A$ admite um autovetor $v$ associado à $\lambda$. Se $v$ é tipo-causal, então segue o resultado. Se $v$ é tipo-espaço, então considere o subespaço $\operatorname{span}(v)^{\perp}$. Tal espaço é Lorentziano e $A$-invariante, pela Proposição 3.21 item iv. Além disso, $\operatorname{dim} \operatorname{span}(v)^{\perp}=n-1$ e, pela hipótese de indução, vale o resultado.

- se $\lambda \in \mathbb{C} \backslash \mathbb{R}$, então consideremos o plano $\pi$ tipo-espaço, proveniente do Lema 3.26. Nesse caso, $c_{A}(x)=p(x) \cdot q(x)$, onde $\operatorname{deg} q(x)=n-2$ e $p(x)=c_{A \mid \pi}$ é irredutível em $\mathbb{R}$ com raízes $\lambda$ e $\bar{\lambda}$. 
Para esse caso específico, vamos mostrar que o resultado vale para $n=3$. Tomemos $\pi^{\perp}, \operatorname{dim} \pi^{\perp}=1$, como $\pi$ é tipo-espaço, então $\pi^{\perp}$ é Lorentziano e $c_{A \mid \pi^{\perp}}(x)=x-\delta$, $\delta \in \mathbb{R}$, portanto $A$ admite um autovetor tipo-tempo.

Para o caso $n>3$, basta aplicar a hipótese de indução em $\pi^{\perp}$ e vale o resultado.

Lema 3.28. Seja $A \in O_{1}(n), n \geq 2$. Se A admite um autovetor tipo-luz $v$ associado a um autovalor $\lambda \neq \pm 1$, então $A$ admite um segundo autovetor tipo-luz $w$ associado ao autovalor $\frac{1}{\lambda}$. Além disso existe um plano A-invariante tipo-tempo $\Sigma$, com $v \in \Sigma$.

Demonstração. Por hipótese, $A$ admite um autovetor tipo-luz, $\log o c_{A}(x)=(x-\lambda) p(x)$, $\operatorname{com} \operatorname{deg} p(x)=n-1$. Novamente faremos por indução sobre $n$. Suponha $n=2$, então $A$ é como descrito na possibilidade ii. da Proposição 3.23, então vale o resultado. Nesse caso, o próprio espaço é o plano $\Sigma$.

Suponha, por hipótese de indução, que o resultado vale para $k<n$ e vamos provar para $k=n$. Sejam $\delta_{2}, \ldots, \delta_{n} \in \mathbb{C}$ as raízes do polinômio $p(x)$, de modo que $c_{A}(x)=(x-\lambda)\left(x-\delta_{2}\right) \ldots\left(x-\delta_{n}\right)$. Logo,

- se $\lambda, \delta_{2}, \ldots, \delta_{n} \in \mathbb{R}$, então $A$ é diagonalizável e $\operatorname{det} A=\lambda \cdot \delta_{2} \ldots \delta_{n}= \pm 1$ (Observação 3.14). Como $\lambda \neq \pm 1$, então deve existir $\delta_{i} \neq \pm 1, \delta_{i} \neq \lambda$, caso contrário $\operatorname{det} A= \pm \lambda$. Então, pela Proposição 3.21 item i., o autovetor $w$ associado à $\delta_{i}$ é tipo-luz e, pela Proposição 3.21 item ii.,

$$
1=\lambda . \delta_{i} \Rightarrow \delta_{i}=\frac{1}{\lambda}
$$

Como $\{v, u\}$ é linearmente independente, pois estão associados a autovalores distintos, então, pela Proposição 3.10, $\operatorname{span}(v, u)$ é o plano $\Sigma$ desejado.

- se existe $i \in\{2, \ldots, n\}$ tal que $\delta_{i} \in \mathbb{C} \backslash \mathbb{R}$, então existe $j \neq i$ tal que $\delta_{j} \in \mathbb{C} \backslash \mathbb{R}$ e $\delta_{j}=\overline{\delta_{i}}$, pelo Lema 3.26. Assim, basta tomar o plano $A$-invariante tipo-espaço $\pi$ e basta aplicar a hipótese de indução para $\left.A\right|_{\pi^{\perp}}$.

Observação 3.29. Uma importante observação deve ser feita ao Lema 3.28 para quando $n=3$. Diante de tal situação onde $n=3$ o resultado não é válido caso alguma das raízes do polinômio característico esteja em $\mathbb{C} \backslash \mathbb{R}$. Se o polinônimo se fatora inteiramente em $\mathbb{R}$, então o resultado segue normalmente.

Porém se o polinômio característico é irredutivel em $\mathbb{R}$, temos que se fatora em $\mathbb{C} d a$ seguinte forma $c_{A}(x)=(x-\lambda)(x-\alpha)(x-\bar{\alpha})$, como visto no Lema 3.26. Assim, podemos ver que A não admite um segundo autovetor, tipo-luz ou não.

Observação 3.30. A Proposição 3.23 mostra que se $n=2$, então A não admite um autovetor tipo-luz associado ao autovalor $\varepsilon$.

Levando em consideração as ressalvas feitas nas Observações 3.29 e 3.30, podemos enunciar o seguinte teorema que caracteriza todos os elementos de $O_{1}(n)$.

Teorema 3.31. Sejam $A \in O_{1}(n)$ e $f_{A} \in \operatorname{Iso}\left(\mathbb{L}^{n}\right)$ a transformação de Lorentz associada à A. Então uma das três possibilidades mutuamente exclusivas ocorre: 
i. A admite um autovetor tipo-tempo. Nesse caso, existe uma base ortonormal $\mathcal{B}$ tal que

$$
M\left(f_{A}, \mathcal{B}\right)=\left[\begin{array}{c|c}
R_{n-1} & 0 \\
\hline 0 & \pm 1
\end{array}\right]
$$

onde $R_{n-1} \in O(n-1)$.

ii. A admite um autovetor tipo-luz associado a autovalor $\lambda \neq \pm 1$. Nesse caso, existe uma base $\mathcal{B}$ tal que

$$
M\left(f_{A}, \mathcal{B}\right)=\left[\begin{array}{c|c}
R_{n-2} & 0 \\
\hline 0 & R
\end{array}\right]
$$

onde $R_{n-2} \in O(n-2)$ e $R \in O_{1}(2)$.

iii. A admite um único autovetor tipo-luz independente associado a autovalor $\lambda= \pm 1$.

Demonstração. Verificamos incialmente que as possibilidades acima são as únicas. Fazendo por indução em $n$, se $n=2$ o resultado segue da Proposição 3.23 e, pela Observação 3.30, a possibilidade iii. não pode acontecer.

Supondo que o resultado valha para $k<n$, veremos quando $k=n$. Pela proposição 3.27 $A$ admite um autovetor causal, $A . v=\lambda v$. Assim, temos que:

- se $v$ é tipo-tempo, então temos a possibilidade $i$. Além disso, a Proposição 3.21 itens $i$. e $i v$. mostra que $\lambda= \pm 1$ e $\operatorname{span}(v)^{\perp}$ é $A$-invariante e tipo-espaço, de modo que basta tomar $\left\{e_{1}, \ldots, e_{n-1}\right\}$ base ortonormal de $\operatorname{span}(v)^{\perp}$ e fazer $\mathcal{B}=\left\{e_{1}, \ldots, e_{n-1}, \frac{v}{\|v\|}\right\}$ para que

$$
M\left(f_{A}, \mathcal{B}\right)=\left[\begin{array}{c|c}
R_{n-1} & 0 \\
\hline 0 & \pm 1
\end{array}\right]
$$

onde $R_{n-1} \in O(n-1)$.

- se $v$ é tipo-luz e

$* \lambda \neq \pm 1$, então temos a possibilidade ii., pelo Lema 3.28. Seja $w$ o segundo autovetor tipo-luz associado ao autovalor $\frac{1}{\lambda}$, então $\operatorname{span}(v, w)$ é um plano tipotempo e $\operatorname{span}(w, v)^{\perp}$ é tipo espaço (Proposição 3.9). De modo que existe base $\mathcal{B}=\mathcal{B}_{1} \cup \mathcal{B}_{2}$, onde $\mathcal{B}_{1}$, base de $\operatorname{span}(v, w)^{\perp}$, e $\mathcal{B}_{2}$, base de $\operatorname{span}(v, w)$, tal que

$$
M\left(f_{A}, \mathcal{B}\right)=\left[\begin{array}{c|c}
R_{n-2} & 0 \\
\hline 0 & R
\end{array}\right],
$$

onde $R_{n-2} \in O(n-2)$ e $R \in O_{1}(2)$.

$* \lambda= \pm 1$, suponha que a possibilidade iii. não ocorra e, assim, exista um autovetor tipo-luz $w$ associado ao autovalor $\delta$, tal que $\{v, w\}$ é lineramente independente. Pela Proposição 3.21 item $i i ., \lambda . \delta=1$, então $\delta=\lambda$. Desse modo,

$$
\langle v \pm w, v \pm w\rangle=\langle A(v \pm w), A(v \pm w)\rangle= \pm 2\langle v, w\rangle
$$

ou seja, ou $v+w$ ou $v-w$ é tipo-tempo, dependendo do valor de $\langle v, w\rangle$. Além disso,

$$
A(v \pm w)=A \cdot v \pm A \cdot w=\varepsilon(v \pm w) .
$$

Portanto, estamos na possibilidade $i$. 
Vamos mostrar agora que os casos são de fato exclusivos. Suponha que ocorra o caso $i$., $\log$ o existe autovetor tipo-tempo $v$ com autovalor $\lambda= \pm 1$ (Proposição 3.21 item $i$.). Suponha, por absurdo, que exista autovetor tipo-luz $w$ com autovalor associado $\delta$. Como $\langle v, w\rangle \neq 0$, pois $\{v, w\}$ é linearmente independente, então

$$
\langle v, w\rangle=\langle A . v, A . w\rangle=\lambda . \delta\langle v, w\rangle
$$

e $\lambda=\delta= \pm 1$, portanto a possibilidade $i i$. não pode ocorrer. Além disso, como $\pi=\operatorname{span}(v, w)$ é um plano tipo-tempo $A$-invariante, então, pela Proposição $3.23,\left.A\right|_{\pi}$ não tem autovetor tipo-luz com autovalor associado \pm 1 . Portanto, a possibilidade $i$ iii. não pode ocorrer caso $i$. ocorra.

Suponha que ocorra a posibilidade $i i$, logo existem autovetores tipo-luz $v$ e $w$ associados aos autovalores $\lambda \neq \pm 1$ e $\frac{1}{\lambda}$ (Proposição 3.28). A possibilidade iii. não pode ocorrer, caso contrário, existe $w$ autovetor tipo-luz com autovalor \pm 1 e, pela Proposição 3.21 item ii., $\pm 1 . \lambda=1 \operatorname{logo} \lambda= \pm 1$. A possibilidade $i$. também não pode ocorrer, pois $\operatorname{span}(w, v)$ é plano tipo-tempo, então $\operatorname{span}(v, w)^{\perp}$ é tipo-espaço e $\left.A\right|_{\text {span }(v, w)}$ está na condição da Proposição 3.23 item $i$.

E, por fim, é claro que se iii. ocorre, então ii. não pode occorer e nem $i$., pela argumentação feita no caso $i . \not$ iii.

Observação 3.32. No mesmo espírito da Observação 3.29, note que se $n=3$ o Teorema acima continua a valer, com a única diferença de que no caso ii. pode não ser possível obter uma base tal que a matriz de $\left.f\right|_{A}$ se escreva daquela forma quando o polinômio característico não se fatorar inteiramente em $\mathbb{R}$.

A possibilidade iii. do Teorema 3.31 é algo pouco intuitivo, aparentemente improvável de acontecer considerando os resultados obtidos nos Lemas 3.27 e 3.28 e até mesmo no próprio Teorema. Porém, é possível obter, de modo razoavelmente simples, uma forma genérica para os elementos de $A \in O_{1}(3)$ tais que $A$ admite um único autovetor tipo-luz associado ao autovalor \pm 1 .

Considere a seguinte base de $\mathbb{L}^{3}, \mathcal{B}=\{u, e, v\}$, onde $u$ e $v$ são vetores tipo-luz linearmente independentes (garantidos pela Proposição 3.10) e e é um vetor tipo-espaço com $\langle e, e\rangle=1$. Além disso, $\langle u, e\rangle=\langle v, e\rangle=0$ e $\langle u, v\rangle=-1$. Vamos supor que $u$ é um autovetor de $A$ associado ao autovalor $\varepsilon= \pm 1$, ou seja, $A . u=\varepsilon u$.

Vamos escrever A.e $=\alpha u+\beta e+\gamma v$. E dessa forma

$0=\langle e, u\rangle=\langle A . e, A . u\rangle=\varepsilon\langle A . e, u\rangle=\langle\alpha u+\beta e+\gamma v, u\rangle=\alpha\langle u, u\rangle+\beta\langle e, u\rangle+\gamma\langle v, u\rangle=-\gamma$.

Ainda,

$$
1=\langle A . e, A . e\rangle=\langle\alpha u+\beta e, \alpha u+\beta e\rangle=\alpha^{2}\langle u, u\rangle+2 \alpha \beta\langle u, e\rangle+\beta^{2}\langle e, e\rangle=\beta^{2} .
$$

Então $\beta=\eta= \pm 1$ e $A \cdot e=\alpha u+\eta e$.

Escrevamos agora $A \cdot v=a u+b e+c v$. Assim,

$$
\langle u, v\rangle=\langle A . u, A . v\rangle=\varepsilon\langle u, a u+b e+c v\rangle=\varepsilon(a\langle u, u\rangle+b\langle u, e\rangle+c\langle u, v\rangle)=\varepsilon c\langle u, v\rangle,
$$

$\log 0=\varepsilon$. Ainda,

$$
\begin{aligned}
& 0=\langle v, e\rangle=\langle A . v, A . e\rangle=\langle a u+b e+\varepsilon v, \alpha u+\eta e\rangle \\
& \quad=a \alpha\langle u, u\rangle+a \eta\langle u, e\rangle+b \alpha\langle e, u\rangle+b \eta\langle e, e\rangle+\varepsilon \alpha\langle v, u\rangle+\varepsilon \eta\langle v, e\rangle=b \eta-\varepsilon \alpha,
\end{aligned}
$$


donde obtemos $b=\varepsilon \eta \alpha$. Além disso,

$$
\begin{aligned}
& 0=\langle v, v\rangle=\langle A . v, A . v\rangle=\langle a u+\varepsilon \eta \alpha e+\varepsilon v, a u+\varepsilon \eta \alpha e+\varepsilon v\rangle \\
& =a^{2}\langle u, u\rangle+\varepsilon \eta \alpha a\langle u, e\rangle+\varepsilon a\langle u, v\rangle+\varepsilon \eta \alpha a\langle e, u\rangle+\alpha^{2}\langle e, e\rangle \\
& +\eta \alpha\langle e, v\rangle+\varepsilon a\langle v, u\rangle+\eta \alpha\langle v, e\rangle+\langle v, v\rangle=-\varepsilon a+\alpha^{2}-\varepsilon a=\alpha^{2}-2 \varepsilon a,
\end{aligned}
$$

então $a=\frac{\varepsilon \alpha^{2}}{2}$ e $A \cdot v=\frac{\varepsilon \alpha^{2}}{2} u+\varepsilon \eta \alpha e+\varepsilon v$. Portanto,

$$
M\left(f_{A}, \mathcal{B}\right)=\left[\begin{array}{ccc}
\varepsilon & \alpha & \frac{\varepsilon \alpha^{2}}{2} \\
0 & \eta & \varepsilon \eta \alpha \\
0 & 0 & \varepsilon
\end{array}\right]
$$

Semelhante ao que fizemos na Seção 3.5.1, vamos considerar a seguinte base ortonormal

$$
\mathcal{B}_{0}=\left\{\frac{1}{\sqrt{2}}(u+v), e, \frac{1}{\sqrt{2}}(v-u)\right\} .
$$

Temos então que

$$
P=\left[\begin{array}{ccc}
\frac{1}{\sqrt{2}} & 0 & -\frac{1}{\sqrt{2}} \\
0 & 1 & 0 \\
\frac{1}{\sqrt{2}} & 0 & \frac{1}{\sqrt{2}}
\end{array}\right] \quad \text { e } \quad P^{-1}=\left[\begin{array}{ccc}
\frac{1}{\sqrt{2}} & 0 & \frac{1}{\sqrt{2}} \\
0 & 1 & 0 \\
-\frac{1}{\sqrt{2}} & 0 & \frac{1}{\sqrt{2}}
\end{array}\right] .
$$

Logo,

$$
M\left(f_{A}, \mathcal{B}_{0}\right)=P^{-1} \cdot M\left(f_{A}, \mathcal{B}\right) . P
$$

e portanto

$$
M\left(f_{A}, \mathcal{B}_{0}\right)=\left[\begin{array}{ccc}
\varepsilon\left(1+\frac{\alpha^{2}}{4}\right) & \frac{\sqrt{2}}{2} \alpha & \varepsilon \frac{\alpha^{2}}{4} \\
\frac{\sqrt{2}}{2} \varepsilon \eta \alpha & \eta & \frac{\sqrt{2}}{2} \varepsilon \eta \alpha \\
-\varepsilon \frac{\alpha^{2}}{4} & -\frac{\sqrt{2}}{2} \alpha & \varepsilon\left(1-\frac{\alpha^{2}}{4}\right)
\end{array}\right] .
$$

Note que, se $\alpha=0$, então $u$ e $v$ são ambos autovetores de $A$ associados a $\varepsilon= \pm 1$. Porém, nesse caso, teremos que

$$
A\left(\frac{1}{\sqrt{2}}(u+v)\right)=\frac{1}{\sqrt{2}}(A \cdot u+A . v)=\varepsilon\left[\frac{1}{\sqrt{2}}(u+v)\right],
$$

ou seja, $\frac{1}{\sqrt{2}}(u+v)$ é autovetor tipo-tempo de $A$ associado a $\varepsilon$, isso confirma o que fizemos na demonstração do Teorema 3.31. 


\section{Capítulo 4}

\section{Geodésicas fechadas incompletas}

Nesse capítulo estudaremos sobre a completude de variedades semi-Riemannianas homogêneas e as diferenças em relação as variedades Riemannianas, falaremos sobre geodésicas fechadas e exibiremos um exemplo de uma tal geodésica (tipo-luz) fechada, mas incompleta.

\subsection{Um pouco sobre ações de grupo e espaço de órbitas}

Considere $\mathbb{R}^{2}$ munido da seguinte métrica $g_{0}=d u \otimes d v+d v \otimes d u$ (ou seja, estamos tomando $\mathbb{L}^{2}$ com coordenadas tipo-luz, como feito na seção 3.5.1). De fato, se tomarmos

$$
u=\frac{e_{1}+e_{2}}{\sqrt{2}} \quad \text { e } \quad v=\frac{e_{1}-e_{2}}{\sqrt{2}}
$$

então, substituindo em $g_{0}$, teremos

$$
\begin{aligned}
g_{0}= & d\left(\frac{e_{1}+e_{2}}{\sqrt{2}}\right) \otimes d\left(\frac{e_{1}-e_{2}}{\sqrt{2}}\right)+d\left(\frac{e_{1}-e_{2}}{\sqrt{2}}\right) \otimes d\left(\frac{e_{1}+e_{2}}{\sqrt{2}}\right) \\
= & \frac{1}{2}\left[\left(d e_{1}+d e_{2}\right) \otimes\left(d e_{1}-d e_{2}\right)+\left(d e_{1}-d e_{2}\right) \otimes\left(d e_{1}+d e_{2}\right)\right] \\
= & \frac{1}{2}\left[d e_{1}^{2}-\left(d e_{1} \otimes d e_{2}\right)+d e_{2} \otimes d e_{1}-\left(d e_{2}^{2}\right)+d e_{1}^{2}\right. \\
& \left.+d e_{1} \otimes d e_{2}-\left(d e_{2} \otimes d e_{1}\right)-d e_{2}^{2}\right] \\
= & d e_{1}^{2}-d e_{2}^{2} .
\end{aligned}
$$

Consideremos a seguinte aplicação $\Phi(u, v)=\left(2 u, \frac{v}{2}\right)$. Observe que dados quaisquer $\left(u_{1}, v_{1}\right)$ e $\left(u_{2}, v_{2}\right)$ em $\mathbb{L}^{2}$, teremos que

$$
\begin{array}{r}
g_{0}\left(\Phi\left(u_{1}, v_{1}\right), \Phi\left(u_{2}, v_{2}\right)\right)=g_{0}\left(\left(2 u_{1}, \frac{v_{1}}{2}\right),\left(2 u_{2}, \frac{v_{2}}{2}\right)\right)=\left(2 u_{1}\right)\left(\frac{v_{2}}{2}\right)+\left(\frac{v_{1}}{2}\right)\left(2 u_{2}\right) \\
=u_{1} v_{2}+v_{1} u_{2}=g_{0}\left(\left(u_{1}, v_{1}\right),\left(u_{2}, v_{2}\right)\right) .
\end{array}
$$

Portanto $\Phi$ é uma isometria para $g_{0}$. É importante notar que $\Phi$ não é uma isometria para a métrica usual Riemanniana $g=d x^{2}+d y^{2}$; de fato, tomando $\left(u_{1}, v_{1}\right)$ e $\left(u_{2}, v_{2}\right)$ em $\mathbb{R}^{2}$ então

$$
g\left(\Phi\left(u_{1}, v_{1}\right), \Phi\left(u_{2}, v_{2}\right)\right)=4 u_{1} u_{2}+\frac{v_{1} v_{2}}{4} \neq u_{1} u_{2}+v_{1} v_{2}=g\left(\left(u_{1}, v_{1}\right),\left(u_{2}, v_{2}\right)\right)
$$


A partir de $\Phi$ formamos o grupo $G=\left\{\Phi^{k} \mid k \in \mathbb{Z}\right\}$. Vamos mostrar, por indução, que $\Phi^{k}(u, v)=\left(2^{k} u, \frac{v}{2^{k}}\right)$. Considere $k \geq 0$, se $k=0$ então $\Phi^{0}=\mathrm{Id}$. Suponha que o resultado seja válido para um $k$ qualquer, vamos mostrar que vale para $k+1$,

$$
\Phi^{k+1}(u, v)=\Phi\left(\Phi^{k}(u, v)\right)=\Phi\left(2^{k} u, \frac{v}{2^{k}}\right)=\left(2\left(2^{k} u\right), \frac{1}{2}\left(\frac{v}{2^{k}}\right)\right)=\left(2^{k+1} u, \frac{v}{2^{k+1}}\right) .
$$

O resultado é análogo para o caso $k<0$.

Nosso objetivo nesse capítulo é mostrar a existência de uma geodésica fechada incompleta no quociente $M / G$, onde $M=\mathbb{R}^{+} \times \mathbb{R}$, munido com a métrica $g_{0}$.

Definição 4.1. Sejam $M$ uma variedade e $G$ um grupo. Uma ação a esquerda de $G$ em $M$ é uma aplicação suave $: G \times M \rightarrow M$, definido por $(g, x) \mapsto g \cdot x$ e satisfazendo:

1. $e \cdot x=x$, para qualquer $x \in M$ e sendo e o elemento neutro do grupo $G$;

2. $g_{1} \cdot\left(g_{2} \cdot x\right)=\left(g_{1} g_{2}\right) \cdot x$, para quaisquer $g_{1}, g_{2} \in G$ e qualquer $x \in M$.

A menos que seja necessário, o símbolo · que representa a ação será omitida.

Definição 4.2. Sejam $M$ uma variedade, $G$ um grupo $e \cdot: G \times M \rightarrow M$ uma ação a esquerda de $G$ em $M$. Para cada $x \in M$, definimos a órbita de $x$ como sendo o conjunto $G \cdot x=\{g \cdot x \mid g \in G\}$.

Definição 4.3. Considere uma ação a esquerda como definida acima, $: G \times M \rightarrow M$. Definimos o grupo de isotropia de $x \in M, G_{x}$, como sendo o conjunto dos elementos de $G$ que fixam $x$, ou seja, $G_{x}=\{g \in G \mid g x=x\}$.

Dizemos que uma ação é livre se para qualquer $x \in M, G_{x}=\{e\}$, ou seja, se para qualquer ponto de $M$ o único elemento de $G$ que o fixa é o elemento neutro.

Definição 4.4. Seja $: G \times M \rightarrow M$ uma ação a esquerda. Dizemos que tal ação é:

- descontínua, se para qualquer sequência de elementos distintos $\left(g_{n}\right) \subset G$ a sequência de pontos em $M,\left(g_{n} x\right)$, não converge, para qualquer $x \in M$.

- propriamente descontínua, se valem as seguintes condições:

i. para quaisquer $x, x^{\prime}$ que não estejam na mesma órbita, ou seja, $x^{\prime} \neq$ gx, para qualquer $g \in G$, existem vizinhanças $U \ni x, U^{\prime} \ni x^{\prime}$ tais que $g U \cap U^{\prime}=\emptyset$, para qualquer $g \in G$;

ii. o grupo de isotropia $G_{x}$ de todo ponto $x \in M$ é finito;

iii. para qualquer $x \in M$, existe $U \ni x$, aberto, tal que $g U \cap U=\emptyset$, para todo $g \in G / G_{x}$.

Para construir o exemplo proposto vamos analisar algumas propriedades da ação de $G=\left\{\Phi^{k} \mid k \in \mathbb{Z}\right\}$, o grupo construído acima, e o conjunto $\mathbb{R}_{*}^{2}=\mathbb{R}^{2} \backslash\{(0,0)\}$.

Exemplo 4.5. Vamos tomar o grupo de isometria em relação à $g=d u^{2}-d v^{2}$, $G=\left\{\Phi^{k} \mid k \in \mathbb{Z}\right\}$, sendo $\Phi^{k}(u, v)=\left(2^{k} u, \frac{v}{2^{k}}\right)$. Observe que se tomarmos a ação de $G$ em $\mathbb{R}^{2}$ tal ação não é livre, já que o grupo de isotropia de $(0,0)$ é todo $G$. De fato,

$$
\Phi^{k}(0,0)=\left(2^{k} \cdot 0, \frac{0}{2^{k}}\right)=(0,0)
$$


para todo $k \in \mathbb{Z}$. Logo $G_{(0,0)}=G$. Entretanto ao tomarmos a ação de $G$ em $\mathbb{R}_{*}^{2}$ teremos uma ação livre, pois

$$
\Phi^{k}(u, v)=(u, v) \Leftrightarrow\left(2^{k} u, \frac{v}{2^{k}}\right)=(u, v) \Leftrightarrow k=0,
$$

para quaisquer $u, v$ não simultaneamente nulos.

$A$ órbita de um ponto $(u, v) \in \mathbb{R}_{*}^{2}$ por $G$ é o conjunto $G \cdot(u, v)=\left\{\Phi^{k}(u, v) \mid k \in \mathbb{Z}\right\}$. Dessa forma, é possivel ver que as órbitas de pontos fora das linhas coordenadas estão contidas em hipérboles, enquanto órbitas de pontos nas linhas coordenadas estão contidas nas próprias linhas coordenadas, embora nunca atinjam a origem. Ou seja,

- se $(u, v) \in \mathbb{R}_{*}^{2}$, com $u, v \neq 0$, então $\Phi^{k}(u, v)=\left(2^{k} u, \frac{v}{2^{k}}\right)$ e a órbita $G \cdot(u, v)=\left\{\left(2^{k} u, \frac{v}{2^{k}}\right) \mid k \in \mathbb{Z}\right\}$.

- se $(u, v) \in \mathbb{R}_{*}^{2}$, com $u=0$ ou $v=0$, então $\Phi^{k}(0, v)=\left(0, \frac{v}{2^{k}}\right)$ ou $\Phi^{k}(u, 0)=\left(2^{k} u, 0\right)$ e a órbita é $G \cdot(0, v)=\left\{\left(0, \frac{v}{2^{k}}\right) \mid k \in \mathbb{Z}\right\}$ ou $G \cdot(u, 0)=\left\{\left(2^{k} u, 0\right) \mid k \in \mathbb{Z}\right\}$.

Como exemplo, na figura abaixo estão representadas alguns pontos das órbitas de $(1,1)$, $(-1,1),(-1,-1)$ e $(1,-1)$.

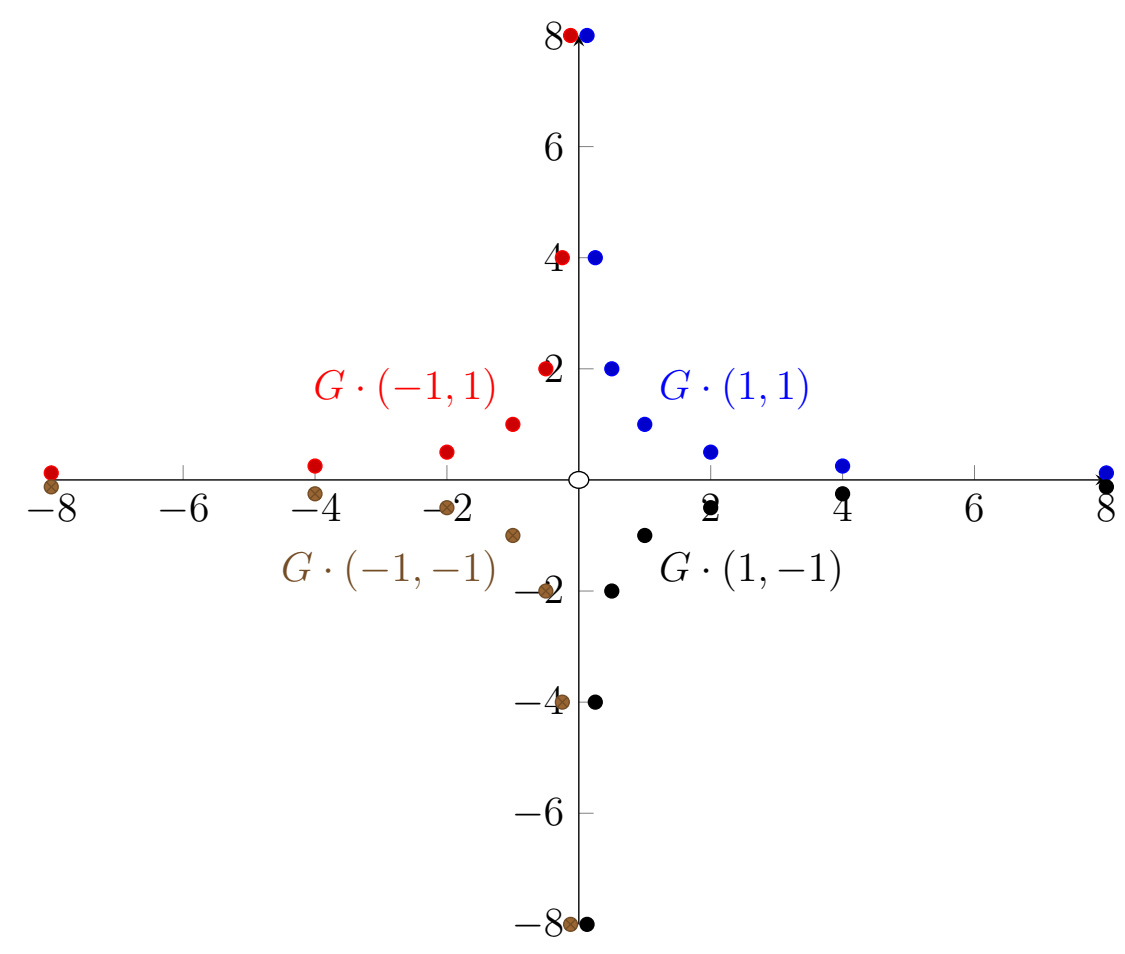

Consideremos uma sequência distinta de elementos de $G,\left(\Phi^{k}\right)_{k \in U} \subset G$, qualquer, sendo $U \subset \mathbb{N}$ um conjunto limitado inferiormente. Dado qualquer ponto $(u, v) \in \mathbb{R}_{*}^{2}$, tome a sequência $\left(\Phi^{k}(u, v)\right)_{k \in U}$. Note que tal sequência está inteiramente contida na órbita de $(u, v)$. Além disso, podemos ver que a órbita de qualquer ponto é um conjunto discreto, logo não há ponto de acumulação; portanto nenhuma sequência $\left(\Phi^{k}(u, v)\right)_{k \in U}$ converge, para qualquer sequência $\left(\Phi^{k}\right)_{k \in U}$ e qualquer $(u, v) \in \mathbb{R}_{*}^{2}$.

Portanto a ação $G \times \mathbb{R}_{*}^{2} \rightarrow \mathbb{R}_{*}^{2}$ é descontínua.

Definição 4.6. Sejam $X$ um espaço topológico e $G$ um grupo de isometrias de $X$, considere a ação natural $G \times X \rightarrow X$. O espaço de órbitas, denotado por $X / G$, é o espaço topológico formado pela órbitas da ação, ou seja, $X / G=\{G \cdot x \mid x \in X\}$. 
Sabemos o que é o espaço de órbitas dado uma ação por isometrias, precisamos entender a topologia desse espaço, em especial, como são os abertos de tal conjunto.

Definição 4.7. Sejam $X$ e $Y$ espaços topológicos. Seja $\rho: X \rightarrow Y$ uma aplicação sobrejetora. Dizemos que $\rho$ é uma aplicação quociente se vale que um subconjunto $U \subset Y e ́$ aberto em $Y$ se, e somente se, $\rho^{-1}(U) \subset X$ é um aberto de $X$.

Definição 4.8. Sejam $X$ um espaço topológico e A um conjunto qualquer. Se $\rho: X \rightarrow A$ é uma aplicação sobrejetora, então existe uma topologia $\mathfrak{T}$ em A na qual $\rho$ é uma aplicação quociente. Definimos $\mathfrak{T}$ como sendo a topologia quociente induzida por $\rho$.

A topologia $\mathfrak{T}$ é definida tomando os subconjuntos $U$ de $A$ tais que $\rho^{-1}(U)$ é um aberto de $X$.

Dessa forma, podemos munir $X / G=\{G \cdot x \mid x \in X\}$ com a topologia quociente. Isso significa que um aberto ao redor de um ponto de $X / G$ é a união de abertos em $X$ dos pontos que compõem tal órbita.

De modo mais explícito, seja $G \cdot x \in X / G$ a órbita de $x$ por $G$, ou seja, $G \cdot x=\{g \cdot x \mid g \in G\}$. Tome $U_{g x}$ um aberto de $X$ ao redor de $g \cdot x$ (dessa forma para $g=e, U_{x}$ é um aberto ao redor do ponto $x$ ). Então um aberto $\mathrm{U}$ de $X / G$ ao redor de $G \cdot x$ é escrito como

$$
\mathbf{U}=\bigcup_{g \in G} U_{g x}
$$

Exemplo 4.9. No exemplo anterior vimos que a ação de $G=\left\{\Phi^{k} \mid k \in \mathbb{Z}\right\}$ sobre $\mathbb{R}_{*}^{2}$ é livre e descontínua. Podemos agora determinar e analisar o seu espaço de órbitas $\mathbb{R}_{*}^{2} / G$ composto pelas órbitas $G \cdot(u, v)$.

Sabemos que os abertos de $\mathbb{R}_{*}^{2} / G$ ao redor de órbitas são uniões de abertos em $\mathbb{R}_{*}^{2}$ ao redor de todos os pontos pertencentes a tal órbita. De modo que os abertos são da seguinte forma:

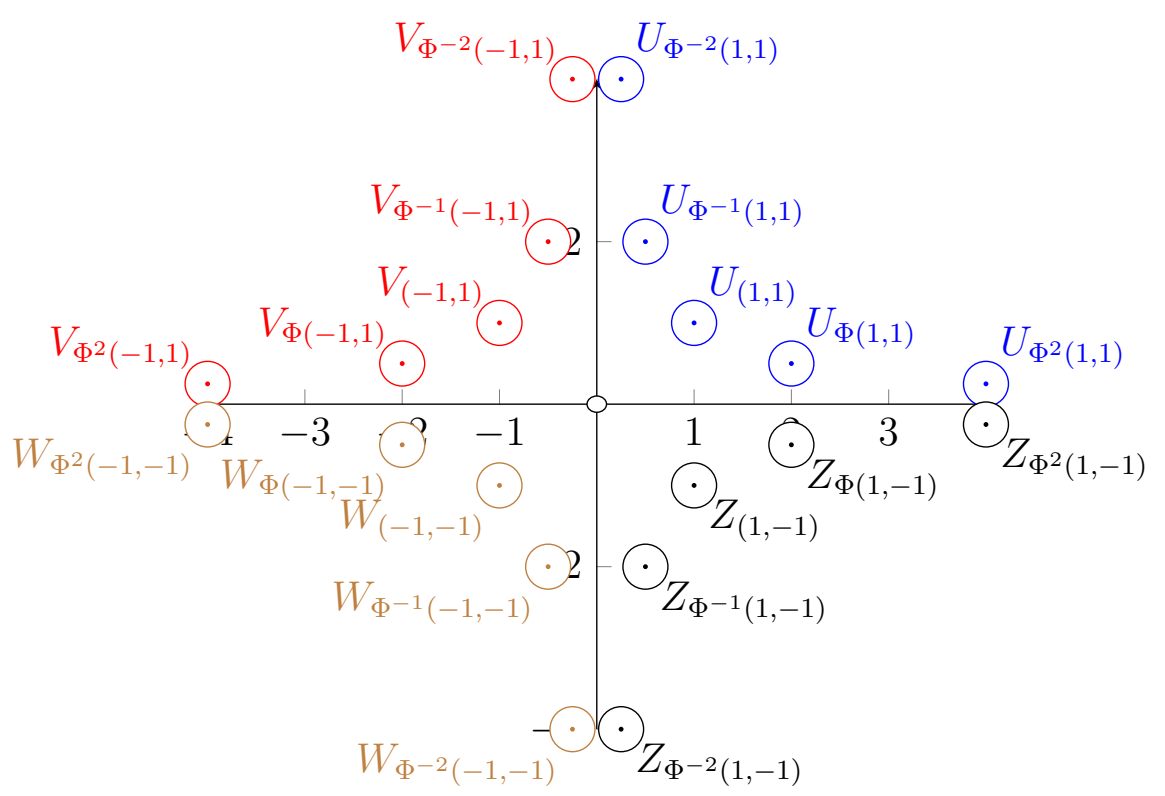

A união dos conjuntos do tipo $U, V, W$ e $Z$, separadamente, formam abertos ao redor das órbitas de $(1,1),(-1,1),(-1,-1) e(1,-1)$, respectivamente.

Agora fica fácil ver que $\mathbb{R}_{*}^{2} / G$ é não-Hausdorff. Para isso, basta considerar as órbitas $G \cdot(1,1)$ e $G \cdot(1,0)$ e os abertos desses pontos em $\mathbb{R}_{*}^{2} / G$; explicitamente, $U_{\Phi^{k}(1,1)}$ e $V_{\Phi^{k}(1,0)}$ 
são abertos de $\mathbb{R}_{*}^{2}$ ao redor de $\Phi^{k}(1,1)$ e $\Phi^{k}(1,0)$, respectivamente, para todo $k \in \mathbb{Z}$, assim

$$
\mathbf{U}=\bigcup_{k \in \mathbb{Z}} U_{\Phi^{k}(1,1)} \quad e \quad \mathbf{V}=\bigcup_{k \in \mathbb{Z}} V_{\Phi^{k}(1,0)}
$$

abertos de $G \cdot(1,1)$ e $G \cdot(1,0)$ em $\mathbb{R}_{*}^{2} / G$, respectivamente. Representados como segue:

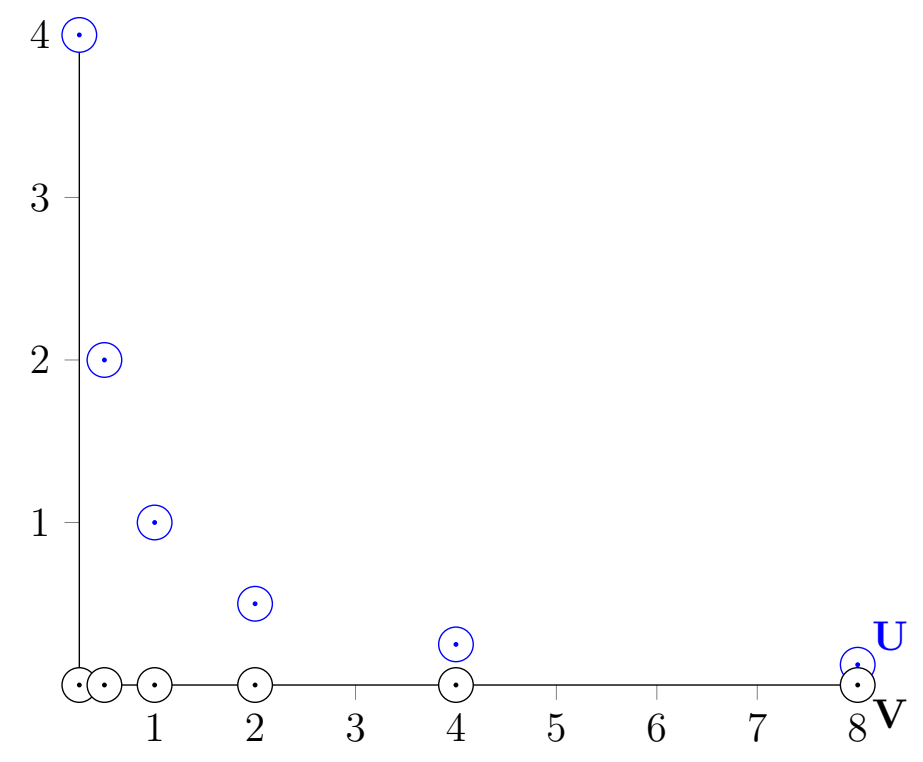

Note que os elementos das órbitas, escritos como $\left(2^{k}, \frac{1}{2^{k}}\right)$ e $\left(2^{k}, 0\right)$, quando $k \rightarrow+\infty$, estão tão pertos quando se queira. Então, para qualquer conjunto de abertos $U_{\Phi^{k}(1,1)}$ e $V_{\Phi^{k}(1,0)}$, existe $k_{0}>0$ tal que $U_{\Phi^{k_{0}(1,1)}} \cap V_{\Phi^{k_{0}(1,0)}} \neq 0$, logo $\mathbf{U} \cap \mathbf{V} \neq 0$. Portanto $\mathbb{R}_{*}^{2} / G$ é nãoHausdorff.

Os exemplos 4.5 e 4.9 exibem um caso simples que evidencia a diferença entre as geometrias Riemanniana e Lorentziana. Como mostrado acima, tomando $\mathbb{R}_{*}^{2}$ com a métrica $g_{0}=d u \otimes d v+d v \otimes d u$ teremos que $\mathbb{R}_{*}^{2} / G$ é uma variedade Lorentziana não-Hausdorff.

Proposição 4.10. Seja $H$ um grupo de isometrias de um espaço métrico $M$. Se a ação $H \times M \rightarrow M$ é descontínua, então é propriamente descontínua.

Demonstração. Note primeiramente que a órbita de qualquer ponto $x \in M$ é fechado em $M$ já que $H \cdot x=\operatorname{Im}(H \times\{x\} \rightarrow M)$. Assim, tome um ponto $y \in M$ tal que $y \notin H \cdot x$ e considere um raio $r$ tal que $2 r<d(y, H \cdot x)$; isso é possível pois $H \cdot x$ é fechado. Basta então tomar os abertos $U \ni x$ e $V \ni y$ ambos de raio $r$, desse modo $g U \cap V=\emptyset$, para qualquer $g \in H$; isso demonstra a condição 1 . da definição de propriamente descontínua.

A segunda condição vem do fato da ação de $H$ em $M$ ser descontínua. De fato, se existesse $x \in M$ tal que o grupo de isotropia $G_{x}=\{g \in H \mid g x=x\}$ fosse infinito existiria então uma sequência de elementos distintos de $H$ tal que $\left(g_{n} x\right)$ converge, pois $g_{n} x=x$.

Assim como na condição $i$, para mostrar a terceira, basta tomar o raio $r$ sendo menor que a distância entre $x$ e $H \cdot x-\{x\}$, que ainda é fechado, e considerar o aberto $U \ni x$ de raio $r$. Então, $g U \cap U=\emptyset$.

Proposição 4.11. Seja $H$ um grupo de transformações diferenciáveis de uma variedade diferenciável $M$. Se a ação $H \times M \rightarrow M$ é livre e propriamente descontínua, então o espaço quociente $M / H$ é uma variedade diferenciável tal que a projeção $\pi: M \rightarrow M / H$ é diferenciável. 
Demonstração. Por hipótese a ação de $H$ em $M$ é livre, logo para qualquer ponto $x \in M$ teremos que $H_{x}=\{e\}$. Tome $x \in M$, como a ação é propriamente descontínua existe $U \ni x$, aberto em $M$, tal que $g U \cap U=\emptyset$ para qualquer $g \in H$. Denotemos $g U$ por $U_{g}$. Dessa forma, como dito antes, teremos que $\mathbf{U}=\bigcup_{g \in H} U_{g}$ é um aberto em $M / H$ ao redor de $H \cdot x$. Note que cada $U_{g}$ é conexo e assim cada um é uma componente conexa de $\pi^{-1}(\mathbf{U})$. Consideremos a projeção $\pi: M \rightarrow M / H, x \mapsto H \cdot x$, do modo como $\mathbf{U}$ foi construído podemos ver que a restrição de cada componente conexa de $\pi^{-1}(\mathbf{U})$ é homeomorfa à $\mathbf{U}$.

Suponhamos agora $\mathbf{U}$ e $\mathbf{V}$ abertos de $M / H$ ao redor de $x$ e $y$, ambos em $M$, tais que $\mathbf{U} \cap \mathbf{V} \neq 0$. Logo, existe uma órbita $G \cdot z \in \mathbf{U} \cap \mathbf{V}$, para algum $z \in M$. Então $g z \in U_{g} \cap V_{g}$, para todo $g \in H$ e, por consequência, $U_{g} \cap V_{g} \neq 0$, para todo $g \in H$.

Agora, para cada $g \in H$, podemos tomar $\psi: U_{g} \rightarrow \mathbb{R}^{n}$ e $\xi: V_{g} \rightarrow \mathbb{R}^{n}$, cartas de $M$. Note que podemos considerar a projeção $\pi$ nas seguintes formas

$$
\begin{aligned}
& \left.\pi\right|_{\mathbf{U}} ^{-1}: \mathbf{U} \rightarrow U_{g} \\
& \left.\pi\right|_{\mathbf{V}} ^{-1}: \mathbf{V} \rightarrow V_{g}
\end{aligned}
$$

e tomamos as funções

$$
\begin{aligned}
& \Psi: \mathbf{U} \rightarrow \mathbb{R}^{n}, \quad \Psi=\left.\psi \circ \pi\right|_{\mathbf{U}} ^{-1} \\
& \Xi: \mathbf{V} \rightarrow \mathbb{R}^{n}, \quad \Xi=\left.\xi \circ \pi\right|_{\mathbf{V}} ^{-1}
\end{aligned}
$$

Logo, na intersecção $\mathbf{U} \cap \mathbf{V}$ teremos

$$
\Psi \circ \Xi^{-1}=\left(\left.\psi \circ \pi\right|_{\mathbf{U}} ^{-1}\right) \circ\left(\left.\xi \circ \pi\right|_{\mathbf{V}} ^{-1}\right)^{-1}=\left.\left.\psi \circ \pi\right|_{\mathbf{U}} ^{-1} \circ \pi\right|_{\mathbf{V}} \circ \xi^{-1}=\psi \circ \xi^{-1},
$$

com

$$
\Psi \circ \Xi^{-1}: \operatorname{Im}(\xi) \rightarrow \operatorname{Im}(\psi)
$$

diferenciável, pois $\psi$ e $\xi$ são cartas de $M$. Portanto, $M / G$ é uma variedade diferenciável.

Ao se tratar de ações por isometrias, como falamos acima, a evolução natural é pensar em variedades tais que todos os pontos podem ser levados a qualquer outro ponto da variedade por isometrias. Ou seja, que a ação é transitiva.

Definição 4.12. Sejam $M$ uma variedade diferenciável e $G$ um grupo de isometrias de $M$. A ação $G \times M \rightarrow M$ é dita transitiva se para quaisquer pontos $x, y \in M$ existe $g \in G$ tal que $g x=y$.

Dessa forma, podemos estabelecer que sendo $(M, g)$ uma variedade semi-Riemanniana e $G$ um grupo de isometrias de $M$, se a ação $G \times M \rightarrow M$ é transitiva dizemos que $M$ é um espaço semi-Riemanniano homogêneo. Os espaços homogêneos ofecerem um exemplo simples que mais uma vez evidencia a diferença entre os casos Riemanniano e semi-Riemanniano. A diferença nesse caso é que tal distinção está na completude das geodésicas, assunto principal desse trabalho.

\subsection{Espaços homogêneos e geodésicas fechadas}

Definição 4.13. Seja $M$ uma variedade diferenciável. Dizemos que $M$ é geodesicamente completa se a exponencial $\exp _{p}$ está definida em todo espaço tangente $\mathrm{T}_{p} M$, para todo $p \in M$. Ou seja, se qualquer geodésica $\gamma: I \rightarrow M$ pode ser definida em toda a reta $\mathbb{R}$.

A completude de geodésicas em variedades Riemannianas é praticamente regida e explicitada pelo Teorema de Hopf-Rinow. Enunciada em [HR31] da seguinte forma: 
Teorema 4.14 (Hopf-Rinow). Seja $M$ uma variedade Riemanniana. Então $M$ é geodesicamente completa se, e somente se, todo conjunto fechado e limitado de $M$ é compacto.

Proposição 4.15. Toda variedade Riemanniana homogênea é completa.

Demonstração. Seja $\rho:[0, b[\rightarrow M$ uma geodésica, vamos mostrar que existe uma extensão de $\rho$ por $b$. Seja $p \in M$ o ponto limite onde $\rho$ não está definida. Considere $B(p, \delta)$ uma vizinhança normal em $p$, ou seja, para qualquer vetor tangente $w \in \mathrm{T}_{p} M$ existe uma geodésica $\mu:\left[0, \delta\left[\rightarrow M\right.\right.$ inteiramente contida em $B(p, \delta)$ tal que $\mu(0)=p$ e $\mu^{\prime}(0)=w$. Tome o ponto $\rho\left(b-\frac{\delta}{2}\right) \in M$ e como $M$ é homogênea, considere a isometria $\phi$ que leva $p$ em $\rho\left(b-\frac{\delta}{2}\right)$; então existe $v \in \mathrm{T}_{p} M$ tal que $d \phi(v)=\rho^{\prime}\left(b-\frac{\delta}{2}\right)$. Assim, $\gamma_{v}$ é a geodésica tal que $\gamma_{v}(0)=p$ e $\gamma_{v}^{\prime}(0)=v$, portanto $\phi \circ \gamma_{v}$ é uma extensão geodésica de $\rho$ passando por $b$.

O resultado acima não é válido para o caso semi-Riemanniano. A demonstração acima não pode ser aplicada pois o vetor $v \in \mathrm{T}_{p} M$ pode ter caráter causal diferente dos vetores tangentes da geodésica $\gamma$, de modo que tal extensão não seria geodésica.

Para o caso semi-Riemanniano, o resultado só é válido caso a variedade seja homogênea e compacta. Tal resultado foi inicialmente provado em [Mar73], mas demonstraremos o resultado seguindo [O’N83].

Teorema 4.16. Seja $M$ uma variedade semi-Riemanniana compacta. Seja $G$ um grupo de Lie agindo transitivamente em $M$ por isometrias. Então $M$ é geodesicamente completa.

Demonstração. Consideremos a geodésica $\gamma:[0, b[\rightarrow M$ e vamos mostrar que é extendível por $b$. Para isso, seja $\left(s_{n}\right)$ uma sequência em $\left[0, b\left[\right.\right.$ tal que $\left(s_{n}\right) \rightarrow b$. Tomando agora a sequência $\gamma\left(s_{n}\right)$ e como $M$ é compacto, então existe uma subsequência $\gamma\left(s_{k}\right) \in M$ tal que $\gamma\left(s_{k}\right) \rightarrow p$, para algum $p \in M$.

Seja $\left\{v_{1}, \ldots, v_{n}\right\}$ uma base de $\mathrm{T}_{p} M$. Para cada vetor $v_{i}$ dessa base podemos estender um campo de Killing $X_{i}$ em $M$, isso é possível pois, por hipotése, $M$ é homogênea e assim podemos transladar cada vetor $v_{i}$ através dos espaços tangentes. Por 2.78, teremos que $\left\langle\gamma^{\prime}(t), X_{i}(\gamma(t))\right\rangle=c_{i}$, para todo $t \in[0, b[$ e $i=1, \ldots, n$.

Afirmação: a sequência $\left(\gamma^{\prime}\left(s_{k}\right)\right) \in \mathrm{T} M$ converge para um vetor $v \in \mathrm{T}_{p} M$.

De fato, como $\left(\gamma\left(s_{k}\right)\right) \rightarrow p$ então, para $k$ suficientemente grande, teremos que $\gamma\left(s_{k}\right) \in \mathcal{X}$, onde $\mathcal{X}$ é uma vizinhança de $p$ tal que $\left\{X_{1}(q), \cdots, X_{n}(q)\right\}$ é base para cada $\mathrm{T}_{q} M$, com $q \in \mathcal{X}$. Se tomarmos $h_{i j}=\left\langle X_{i}, X_{j}\right\rangle$, então det $h \neq 0$ e, supondo $\gamma^{\prime}\left(s_{k}\right)=\sum a^{i} X_{i}$,

$$
\left\langle\gamma^{\prime}\left(s_{k}\right), X_{j}\right\rangle=c_{j} \Rightarrow\left\langle\sum a^{i} X_{i}, X_{j}\right\rangle=c_{j} \Rightarrow \sum a^{i} h_{i j}=c_{j},
$$

gerando o seguinte sistema

$$
\left\{\begin{array}{ccc}
a^{1} h_{11}+\cdots+a^{n} h_{n 1} & = & c_{1} \\
\vdots & & \vdots \\
a^{1} h_{1 n}+\cdots+a^{n} h_{n n} & = & c_{n}
\end{array}\right.
$$

ou ainda

$$
\left[\begin{array}{ccc}
h_{11} & \cdots & h_{1 n} \\
\vdots & \ddots & \vdots \\
h_{n 1} & \cdots & h_{n n}
\end{array}\right]\left[\begin{array}{c}
a^{1} \\
\vdots \\
a^{n}
\end{array}\right]=\left[\begin{array}{c}
c_{1} \\
\vdots \\
c_{n}
\end{array}\right]
$$


$\log 0$

$$
\left\{\begin{array}{ccc}
a^{1} & = & h^{11} c_{1}+\cdots+h^{1 n} c_{n} \\
\vdots & & \vdots \\
a^{n} & = & h^{n 1} c_{1}+\cdots+h^{n n} c_{n}
\end{array}\right.
$$

Portanto,

$$
a^{i}=\sum h^{i j} c_{j} \quad \text { e } \quad \gamma^{\prime}\left(s_{k}\right)=\sum h^{i j} c_{j} X_{i} .
$$

Dessa forma, podemos ver que $\left(\gamma^{\prime}\left(s_{k}\right)\right)$ converge, pois, mais explicitamente,

$$
\gamma^{\prime}\left(s_{k}\right)=\sum h^{i j}\left(\gamma\left(s_{k}\right)\right) c_{j} X_{i}\left(\gamma\left(s_{k}\right)\right) \rightarrow \sum h^{i j}(p) c_{j} v_{i}=v \in \mathrm{T}_{p} M .
$$

Pela Proposição 2.64, $\gamma^{\prime}:[0, b[\rightarrow$ T $M$ é uma curva integral do campo geodésico $G$. E pela Proposição 2.72, $\gamma^{\prime}$ tem uma extensão através de $b$ como curva integral. Portanto, de novo pela 2.64, essa extensão da curva integral projeta por $\pi$ uma geodésica em $M$ que estende $\gamma$.

Muitos exemplos podem ser construídos afim de mostrar que uma variedade homogênea e não compacta é incompleta. A seguir construiremos um desses exemplos usando curvas fechadas, mas outros exemplos podem ser construídos de modo sistemático, deixamos [DI93] como referência.

Vamos começar vendo algumas propriedades de geodésicas fechadas em variedades semiRiemannianas.

Definição 4.17. Sejam $(M, g)$ uma variedade Semi-Riemanniana e $\gamma: I \rightarrow M$ uma geodésica não constante. Dizemos que $\gamma$ é fechada se existem $a, b \in I$ e $\lambda \in \mathbb{R}$ tal que $\gamma(a)=\gamma(b) e$

$$
\gamma^{\prime}(b)=\lambda \gamma^{\prime}(a)
$$

Em particular, se $\lambda=1$ dizemos que $\gamma$ é periódica.

Note que se $\gamma: I \rightarrow M$ é uma geodésica fechada, então $\lambda$ não pode ser menor que 0 . De fato, suponha que $\lambda<0$. Sem perda de generalidade tomemos $\lambda=-1$ e então $\gamma^{\prime}(b)=-\gamma^{\prime}(a)$. Temos assim a seguinte situação:

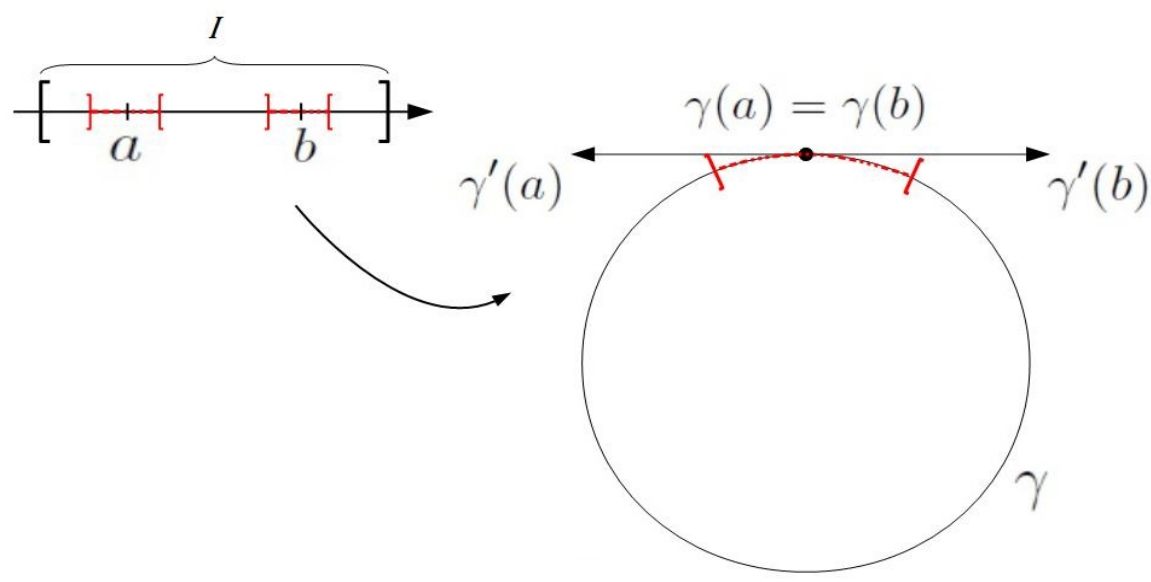

Figura 4.1: Representação de $\gamma \operatorname{com} \gamma^{\prime}(b)=-\gamma^{\prime}(a)$

Pela unicidade da geodésica, existe $\varepsilon>0$ tal que $\gamma(a+t)=\gamma(b-t)$, para todo $t \in[-\varepsilon, \varepsilon]$. Agora, vamos escrever $\gamma(a)=\gamma(b)=p$ e $\gamma^{\prime}(a)=-\gamma^{\prime}(b)=v$. Tomemos então $\alpha$ e $\beta$, as 
geodésicas definidas com as seguintes condições iniciais

$$
\left\{\begin{array} { c } 
{ \alpha ( 0 ) = p } \\
{ \alpha ^ { \prime } ( 0 ) = v }
\end{array} \quad \text { e } \quad \left\{\begin{array}{c}
\beta(0)=p \\
\beta^{\prime}(0)=-v
\end{array}\right.\right.
$$

Obtemos assim, uma situação semelhante a seguinte:

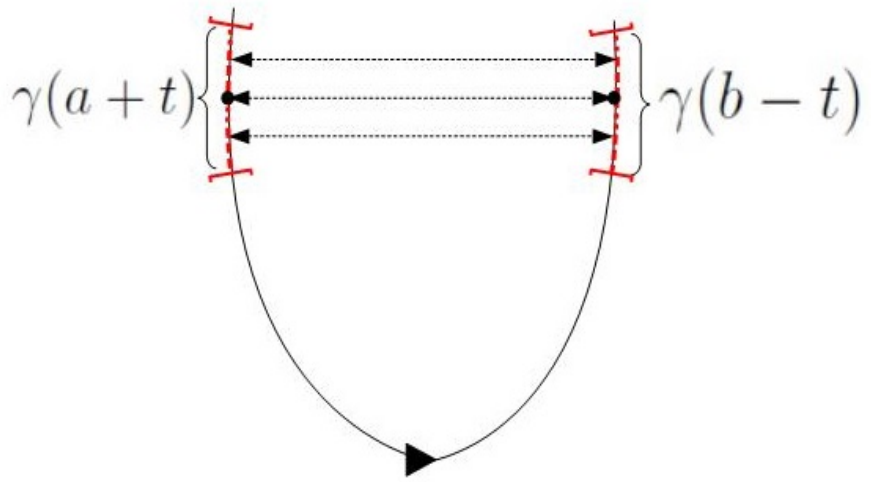

(a)

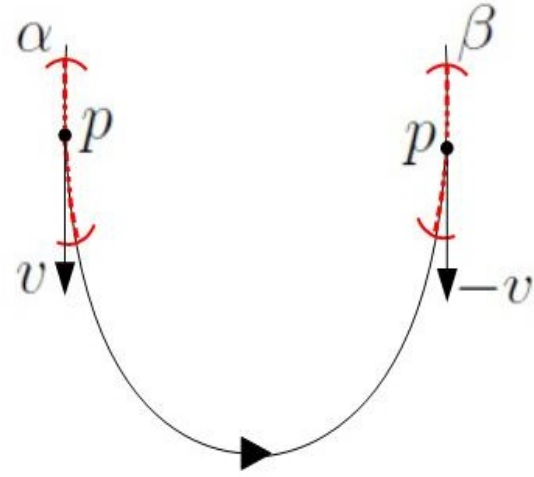

(b)

Figura 4.2: Representação "aberta" de $\gamma$ e reparametrização por $\alpha$ e $\beta$

Assim, podemos definir $\alpha$ e $\beta$ em uma intervalo $]-\delta, \delta[$ e mais uma vez pela unicidade da geodésica temos, ajustando os intervalos, se necessário, com $t \in[0, \delta[$ :

$$
\left\{\begin{array}{l}
\alpha(s)=\gamma(a+s) \\
\beta(s)=\gamma(b-s)
\end{array}\right.
$$

O que implica que existe $s_{0} \in[a, b]$ tal que $\gamma^{\prime}\left(s_{0}\right)=-\gamma^{\prime}\left(s_{0}\right)$.

Proposição 4.18. Seja $\gamma: I \rightarrow M$ uma geodésica fechada em uma variedade semiRiemanniana $(M, g)$.

i. Se $\gamma$ não é do tipo-luz, então é periódica.

ii. $\gamma$ é completa se, e somente se, é periódica.

Demonstração. i. Como $\gamma$ é fechada, $\gamma^{\prime}(b)=\lambda \gamma^{\prime}(a)$, logo

$$
g\left(\gamma^{\prime}(b), \gamma^{\prime}(b)\right)=\lambda^{2} g\left(\gamma^{\prime}(a), \gamma^{\prime}(a)\right)
$$

e como $\gamma$ não é do tipo-luz temos $g\left(\gamma^{\prime}, \gamma^{\prime}\right) \neq 0$. Portanto, $\lambda^{2}=1$ e então $\lambda=1$.

ii. $(\Leftarrow)$ Por hipótese, $\gamma^{\prime}(b)=\gamma^{\prime}(a)$, ou seja, $\gamma$ é periódica e além disso $\gamma$ tem período $T=b-a$, logo é possível obter uma extensão geodésica $\tilde{\gamma}$ definida em $\mathbb{R}$ tal que $\tilde{\gamma}(t)=\gamma(t+n T)$, para qualquer $t \in \mathbb{R}$ e qualquer $n \in \mathbb{N}$.

$(\Rightarrow)$ Suponha que $\lambda<1$, vamos mostrar que $\gamma$ é incompleta na diração de $+\infty$ (de modo análogo é possível mostar que se $\lambda<1$, então $\gamma$ é incompleta na direção de $-\infty)$.

Seja $b_{1}:=b$ e $T=b-a$. Como $\gamma^{\prime}\left(b_{1}\right)=\lambda \gamma^{\prime}(a)$, então consideremos uma extensão geodésica $\gamma_{1}$ de $\gamma$ com domínio $\left[a, b_{2}\right]$, onde $b_{2}:=b_{1}+\frac{T}{\lambda}$, tal que $\gamma_{1}(a)=\gamma_{1}\left(b_{1}\right)=\gamma_{1}\left(b_{2}\right)$ e $\gamma_{1}^{\prime}\left(b_{2}\right)=\lambda \gamma_{1}^{\prime}\left(b_{1}\right)=\lambda^{2} \gamma_{1}^{\prime}(a)$. Da mesma forma, estendemos de modo que $b_{i+1}=b_{i}+\frac{T}{\lambda^{i}}$ e 
$\gamma_{i}^{\prime}\left(b_{i+1}\right)=\lambda^{i+1} \gamma_{i}^{\prime}(a)$. Assim, considerando essa sequência

$$
\lim _{i \rightarrow \infty} b_{i}=b+\left[\sum_{i=0}^{\infty} \frac{T}{\lambda}\left(\frac{1}{\lambda^{i}}\right)\right]=b+\frac{T}{\lambda-1}<+\infty,
$$

de modo que não possível definir $\gamma\left(b+\frac{T}{\lambda-1}\right)$. Portanto $\gamma$ não pode ser estendida até $+\infty$.

Como vimos anteriormente, uma curva tal que cada volta é feita em tempos distintos, é uma curva não periódica e, por consequência, não é completa. Assim, queremos agora construir uma geodésica fechada tipo-luz que mostraremos ser incompleta mais tarde. Para essa construção tomaremos a variedade $\mathbb{R}^{+} \times \mathbb{R}$ e a partir dela construir um cilindro onde definiremos a geodésica descrita.

Considerando o grupo $G=\left\{\Phi^{k} \mid k \in \mathbb{Z}\right\}$ definido como na seção anterior, com as isometrias $\Phi^{k}(u, v)=\left(2^{k} u, \frac{v}{2^{k}}\right)$, vamos mostrar que o espaço de órbitas $\left(\mathbb{R}^{+} \times \mathbb{R}\right) / G$ pode ser visto como a faixa em $\mathbb{R}^{2}$ :

$$
C=\left\{(x, y) \in \mathbb{R}^{2} \mid x \in[1,2]\right\} .
$$

Para isso, precisamos mostrar que os pontos de fora de $C$ caem em $C$ para algum $k \in$ $\mathbb{Z}$ e $\Phi^{k} \in G$, dessa forma podemos tomar os elementos de $C$ como os representantes da órbitas. Observe que podemos identificar as retas $x=1$ e $x=2$ pois $\Phi(1, y)=\left(2, \frac{y}{2}\right)$, logo desconsideramos esses pontos momentaneamente. Em resumo, iremos mostrar que para qualquer órbita de $\left(\mathbb{R}^{+} \times \mathbb{R}\right) / G$ existe um único elemento pertencente a $C$, com exceção das retas descritas acima.

Vamos tomar um ponto $(u, v) \in \mathbb{R}^{+} \times \mathbb{R}, \operatorname{com} u \neq 1,2$. Então $\Phi^{k}(u, v)=\left(2^{k} u, \frac{v}{2^{k}}\right)$, para $k \in \mathbb{Z}$. Para que $\left(2^{k} u, \frac{v}{2^{k}}\right) \in C$ precisamos que

$$
2^{k} u>1 \Leftrightarrow 2^{k}>\frac{1}{u} \Leftrightarrow k \ln 2>-\ln u \Leftrightarrow k>-\frac{\ln u}{\ln 2}
$$

e ainda

$$
2^{k} u<2 \Leftrightarrow 2^{k-1}<\frac{1}{u} \Leftrightarrow(k-1) \ln 2<-\ln u \Leftrightarrow k<-\frac{\ln u}{\ln 2}+1 .
$$

Logo, $\left(2^{k} u, \frac{v}{2^{k}}\right) \in C$ se, e somente se,

$$
-\frac{\ln u}{\ln 2}<k<-\frac{\ln u}{\ln 2}+1
$$

Portanto existe um único $k \in \mathbb{Z}$ tal que $\Phi^{k}(u, v) \in C$.

Note que os valores de $k$ mudam dependendo do ponto em $\mathbb{R}^{+} \times \mathbb{R}$, de modo que

$$
\left\{\begin{array}{l}
\frac{\ln u}{\ln 2}<0, \quad \text { se } u<1 \\
\frac{\ln u}{\ln 2}>1, \quad \text { se } u>2
\end{array}\right.
$$

e assim, $k>0$, se $u<1$, e $k<0$, se $u>2$. Os pontos $(u, v)$ com $1<u<2$ já estão em $C$, 
ainda

$$
2^{k}<2^{k} u<2^{k+1} \Rightarrow \begin{cases}2^{k} u>2, & \text { se } k \geq 1 \\ 2^{k} u<1, & \text { se } k<1\end{cases}
$$

$\operatorname{logo} \Phi^{k}(u, v) \notin C$, para $1<u<2$ e qualquer $k \in \mathbb{Z}$.

Para obtermos a variedade $M$ basta que tomemos a seguinte relação $(1, y) \sim\left(2, \frac{y}{2}\right)$, o que esclarece o motivo por termos deixado de lado as retas $x=1$ e $x=2$, para que as usassemos como pontos de colagem agora. Perceba que a relação $\sim$ consiste em simplesmente aplicar $\Phi$ à reta $x=1$, que invarialvemente cairá na reta $x=2$.

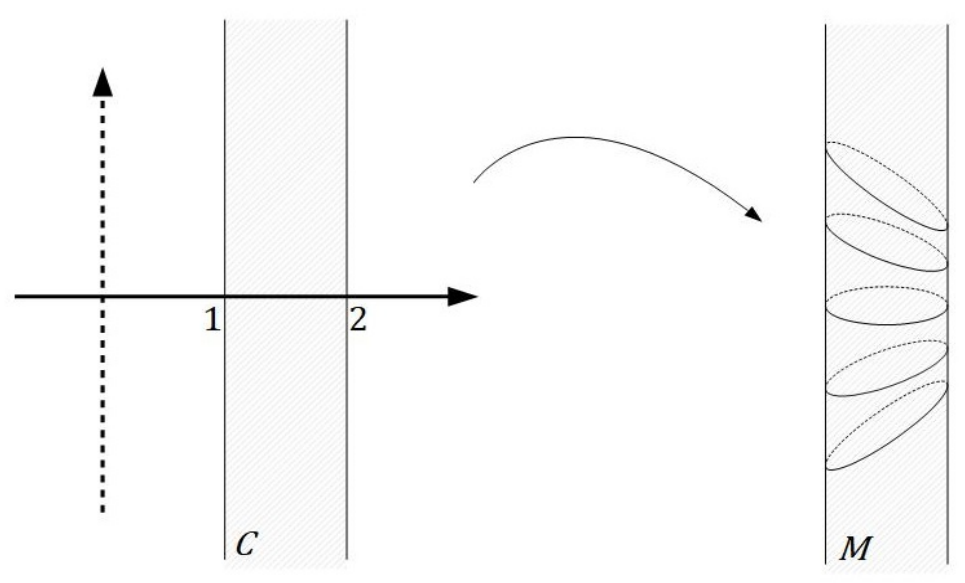

Figura 4.3: Transformação de $C$ em $M$ a partir do grupo $G$ e relação de equivalência $~$

Fizemos essa construção para mostrar que $C / \sim$ é o cilindro $M$. Consideremos as seguintes curvas

$$
\left\{\begin{array}{cc}
\tilde{\gamma}(s)=(-s, 0), & \text { geodésica em } \mathbb{R}^{+} \times \mathbb{R} \\
\gamma, & \text { projeção da geodésica } \gamma \text { em } \mathrm{C}=\left(\mathbb{R}^{+} \times \mathbb{R}\right) / \mathrm{G} .
\end{array}\right.
$$

O que significa que os ponto de $\gamma$ são órbitas de pontos de $\tilde{\gamma}$ e é claro que $\gamma$ também é geodésica já que $\tilde{\gamma}$ o é. Além disso, $\gamma$ é uma geodésica incompleta, pois é não periódica. De fato, ao considerar a geodésica $\tilde{\gamma}$, estamos tomando $s<0$. Dessa forma, tome $s_{1}<0$, algum instante dentro do domínio de $\tilde{\gamma}$, e seja $s_{2}=\frac{s_{1}}{2}$ de modo que $T=s_{2}-s_{1}>0$. Vamos olhar por um momento o que foi dito sob a perspectiva de $\gamma$. Temos que

$$
\tilde{\gamma}\left(s_{2}\right)=\tilde{\gamma}\left(\frac{s_{1}}{2}\right)=\left(-\frac{s_{1}}{2}, 0\right)=\Phi^{-1}\left(-s_{1}, 0\right)=\Phi^{-1}\left(\tilde{\gamma}\left(s_{1}\right)\right),
$$

onde $\Phi^{-1}(u, v)=\left(\frac{u}{2}, v\right), \operatorname{com} \Phi^{-1} \in G$. Isso significa que $\tilde{\gamma}\left(s_{2}\right) \in G \cdot \tilde{\gamma}\left(s_{1}\right)$, ou seja, $\gamma\left(s_{1}\right)=\gamma\left(s_{2}\right)$.

Analogamente, seja $s_{3}=\frac{s_{2}}{2}, \operatorname{com} s_{3}-s_{2}=\frac{s_{2}}{2}-\frac{s_{1}}{2}=\frac{T}{2}$. Então

$$
\tilde{\gamma}\left(s_{3}\right)=\left(-\frac{s_{1}}{4}, 0\right)=\frac{1}{4} \tilde{\gamma}\left(s_{1}\right)=\Phi^{-2}\left(\tilde{\gamma}\left(s_{1}\right)\right)
$$

assim, $\gamma\left(s_{3}\right)=\gamma\left(s_{2}\right)=\gamma\left(s_{1}\right)$. Em resumo, temos a seguinte situação

$\rightarrow$ O percurso de $\gamma\left(s_{1}\right)$ até $\gamma\left(s_{2}\right)$ demorou uma quantidade $T$ de tempo; 
$\rightarrow$ O percurso de $\gamma\left(s_{2}\right)$ até $\gamma\left(s_{3}\right)$ demorou uma quantidade $T / 2$ de tempo;

$\rightarrow$ Como $\gamma\left(s_{3}\right)=\gamma\left(s_{2}\right)=\gamma\left(s_{1}\right)$, então é claro que $\gamma^{\prime}\left(s_{3}\right)=\lambda \gamma^{\prime}\left(s_{1}\right)$, com $\lambda>1$.

E, de fato, a $k$-ésima volta completa de $\gamma$ leva $T / 2^{k-1}$ de tempo para ser efetuada e assim $\gamma^{\prime}\left(s_{k+1}\right)=\lambda^{k-1} \gamma\left(s_{1}\right)$, com $\lambda>1$.

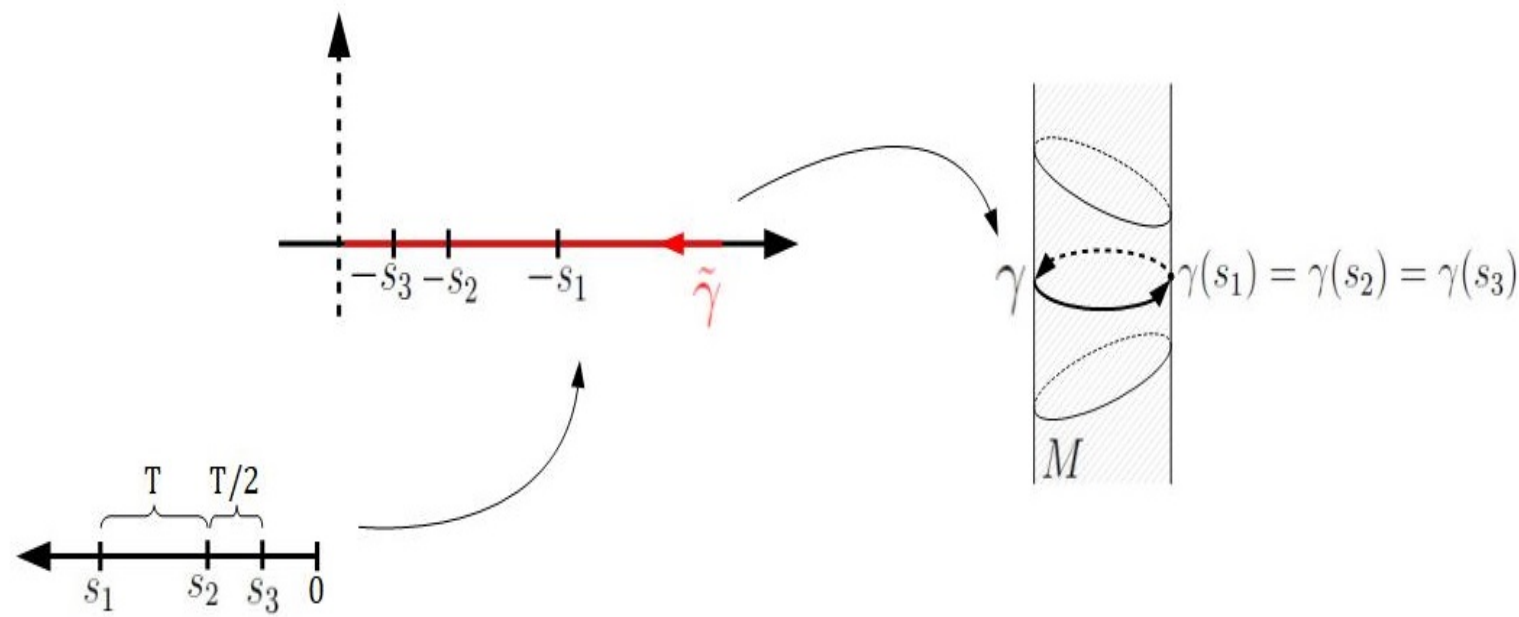

Figura 4.4: Transformação de $\tilde{\gamma}$ em $\gamma$ pelo grupo $G$ e relação de equivalência $\sim$

Portanto, pela Proposição 4.18, $\gamma$ é incompleta. O que prova que somente a homogeneidade não é suficiente para garantir que a variedade é geodesicamente completa. 


\section{Capítulo 5}

\section{Completude por caráter causal}

\subsection{Métricas conformes e pré-geodésicas}

Definição 5.1. Sejam $(M, g)$ e $(N, h)$ duas variedades semi-Riemannianas e $\Phi: M \rightarrow N$ um difeomorfismo. Dizemos que $\Phi$ é uma transformação conforme se existe uma função $\Lambda \in \mathcal{F}(M)$ tal que

$$
\begin{aligned}
& \Phi^{*} \cdot h=\Lambda \cdot g, \\
& \begin{aligned}
& \text { ou seja, } h\left(d \Phi_{p}\left(X_{p}\right), d \Phi_{p}\left(Y_{p}\right)\right)= \Lambda(p) g\left(X_{p}, Y_{p}\right), \text { para qualquer } p \in M \text { e quaisquer } \\
& X_{p}, Y_{p} \in \mathrm{T}_{p} M .
\end{aligned}
\end{aligned}
$$

Observação 5.2. 1. É possível definir a transformação conforme local caso $\Phi$ seja um difeomorfismo local entre $M$ e $N$.

2. Note que se $\Lambda \equiv 1$, então $\Phi$ é uma isometria.

Definição 5.3. Sejam $g$ e $g^{*}$ duas métricas semi-Riemannianas em uma variedade M. Dizemos que $g$ e $g^{*}$ são conformes se a função identidade Id $:(M, g) \rightarrow\left(M, g^{*}\right)$ é uma transformaçãa conforme. Ou seja, $g$ e $g^{*}$ são conformes se existe $\Lambda \in \mathcal{F}(M)$ tal que $g^{*}=\Lambda \cdot g$.

Observação 5.4. Sendo $g$ e $g^{*}$ métricas conformes, por vezes diremos que $g^{*}$ é conforme $\grave{a} g$ quando $g^{*}=\Lambda \cdot g$.

Um primeiro resultado interessante que surge dessa definição vem diretamente do Teorema 2.79 .

Corolário 5.5. Sejam g e $g^{*}$ duas métricas semi-Riemannianas em uma variedade $M$ e suponha que g é uma métrica indefinida. Então $g^{*}$ é conforme à $g$ se, e somente se, os vetores tipo-luz para $g$ são vetores tipo-luz para $g^{*}$.

Demonstração. Basta aplicar o Teorema 2.79 em cada $p \in M$ e nas métricas $g_{p}$ e $g_{p}^{*}$.

Outro resultado de grande relevância quando lidamos com métricas conformes e usaremos durante a seção é sobre a relação entre as conexões de Levi-Civita de tais métricas.

Teorema 5.6. Sejam $g$ e $g^{*}$ métricas semi-Riemannianas conformes em uma variedade de dimensão $n, M$, ou seja, $g^{*}=\Lambda \cdot g$, com $\Lambda \in \mathcal{F}(M)$ e $\Lambda>0$. E sejam $\nabla$ e $\nabla^{*}$ as conexões de Levi-Civita associadas à $g$ e $g^{*}$ respectivamente. Então

$$
2 \Lambda \nabla_{X}^{*} Y=2 \Lambda \nabla_{X} Y+X(\Lambda) Y+Y(\Lambda) X-g(X, Y) \nabla \Lambda
$$


para quaisquer $X, Y \in \mathfrak{X}(M)$.

Demonstração. Diretamente da fórmula de Koszul, se $X, Y, W \in \mathfrak{X}(M)$,

$$
\begin{aligned}
2 g^{*}\left(\nabla_{X}^{*} Y, W\right)=X g^{*}(Y, W)+Y g^{*}(W, X)-W g^{*}(X, Y) & \\
& -g^{*}(X,[Y, W])+g^{*}(Y,[W, X])+g^{*}(W,[X, Y]) .
\end{aligned}
$$

Como as métricas são conformes

$$
\begin{gathered}
2 \Lambda g\left(\nabla_{X}^{*} Y, W\right)=\quad X(\Lambda g(Y, W))+Y(\Lambda g(W, X))-W(\Lambda g(X, Y)) \\
-\Lambda[g(X,[Y, W])-g(Y,[W, X])-g(W,[X, Y])] \\
=\quad X(\Lambda) g(Y, W)+\Lambda X g(Y, W)+Y(\Lambda) g(W, X) \\
\quad+\Lambda Y g(W, X)-W(\Lambda) g(X, Y)-\Lambda W g(X, Y) \\
\quad-\Lambda[g(X,[Y, W])-g(Y,[W, X])-g(W,[X, Y])] \\
=\quad X(\Lambda) g(Y, W)+Y(\Lambda) g(W, X)-W(\Lambda) g(X, Y) \\
\quad+\Lambda[X g(Y, W)+Y g(W, X)-W g(X, Y) \\
\\
\quad-g(X,[Y, W])+g(Y,[W, X])+g(W,[X, Y])] \\
=\quad X(\Lambda) g(Y, W)+Y(\Lambda) g(W, X)-W(\Lambda) g(X, Y) \\
\quad+2 \Lambda g\left(\nabla_{X} Y, W\right) .
\end{gathered}
$$

Note que $W(\Lambda) g(X, Y)=g(g(X, Y) \nabla \Lambda, W)$. De fato, seja $\left\{\partial_{1}, \ldots, \partial_{n}\right\}$ e suponha que $X=\sum_{i} X^{i} \partial_{i}, Y=\sum_{j} Y^{j} \partial_{j}$ e $W=\sum_{k} W^{k} \partial_{k}, \operatorname{com} \nabla \Lambda=\sum_{p, q} g^{p q} \partial_{p}(\Lambda) \partial_{q}$. Então

$$
\begin{aligned}
g(g(X, Y) \nabla \Lambda, W) & =g\left(\sum_{i, j, p, q} X^{i} Y^{j} g_{i j} g^{p q} \partial_{p}(\Lambda) \partial_{q}, \sum_{k} W^{k} \partial_{k}\right) \\
& =\sum_{i, j, k, p, q} X^{i} Y^{j} W^{k} \partial_{p}(\Lambda) g_{i j} g^{p q} g_{q k} \\
& =\sum_{i, j, k} X^{i} Y^{j} W^{k} \partial_{k}(\Lambda) g_{i j} \\
& =\left[\sum_{k} W^{k} \partial_{k}(\Lambda)\right] g\left(\sum_{i} X^{i} \partial_{i}, \sum_{j} Y^{j} \partial_{j}\right) \\
& =W(\Lambda) g(X, Y),
\end{aligned}
$$

pois $\sum_{q} g^{p q} g_{q k}=\delta_{p k}$. Logo

$$
\begin{aligned}
2 \Lambda g\left(\nabla_{X}^{*} Y, W\right)= & X(\Lambda) g(Y, W)+Y(\Lambda) g(W, X) \\
& \quad-g(g(X, Y) \nabla \Lambda, W)+2 \Lambda g\left(\nabla_{X} Y, W\right) \\
= & g\left(2 \Lambda \nabla_{X} Y+X(\Lambda) Y+Y(\Lambda) X-g(X, Y) \nabla \Lambda, W\right),
\end{aligned}
$$

para todo $W \in \mathfrak{X}(M)$. Portanto,

$$
2 \Lambda \nabla_{X}^{*} Y=2 \Lambda \nabla_{X} Y+X(\Lambda) Y+Y(\Lambda) X-g(X, Y) \nabla \Lambda
$$


Definição 5.7. Seja $\gamma$ uma curva em $M$. Dizemos que $\gamma$ é uma pré-geodésica se existe uma reparametrização $\tilde{\gamma}$ tal que seja uma geodésica.

Lema 5.8. Sejam $M$ uma variedade munida de uma métrica semi-Riemanniana $g$ e $\gamma: I \rightarrow M$ uma curva tal que $\gamma^{\prime}(t) \neq 0$, para todo $t \in I$. Se

$$
\frac{D \gamma^{\prime}}{d t}=f(t) \gamma^{\prime}(t)
$$

para todo $t \in I$ e para alguma função $f: I \rightarrow \mathbb{R}$, então $\gamma$ é uma pré-geodésica de $g$.

Demonstração. Considere a reparametrização $\tilde{\gamma}(s)=\gamma(t(s))$, então derivando em $s$, $\tilde{\gamma}^{\prime}(s)=t^{\prime}(s) \gamma^{\prime}(t(s))$ e tomando a derivada covariante nos campos $\tilde{\gamma}^{\prime}$ e $\gamma^{\prime}$

$$
\frac{D}{d s} \tilde{\gamma}^{\prime}=t^{\prime \prime}(s) \gamma^{\prime}(t(s))+t^{\prime}(s)^{2} \frac{D}{d t} \gamma^{\prime}(t(s))=\left[t^{\prime \prime}(s)+t^{\prime}(s)^{2} f(t(s))\right] \gamma^{\prime}(t(s))
$$

onde a última igualdade vem da equação 5.2. Então $\tilde{\gamma}$ é uma geodésica se, e somente se, $t^{\prime \prime}(s)+t^{\prime}(s)^{2} f(t(s))=0$, para todo $s \in J$.

Vamos mostrar agora que se vale 5.2, então sempre é possível obter tal reparametrização. Considere $\tilde{\gamma}(s)=\gamma(t(s))$, onde $t: J \rightarrow I$ é a função tal que sua inversa $s: I \rightarrow J$ possui derivada

$$
s^{\prime}(t)=s^{\prime}\left(t_{0}\right) \cdot e^{\int_{t_{0}}^{t} f(x) d x},
$$

para algum $t_{0} \in \mathbb{R}$ fixado e $s^{\prime}\left(t_{0}\right)>0$. Vamos mostrar que $t^{\prime \prime}(s)+f(t(s)) \cdot t^{\prime}(s)^{2}=0$.

De fato, como $t$ e $s$ são inversas, então

$$
t^{\prime}(s)=\frac{1}{s^{\prime}(t(s))} .
$$

Derivando teremos que

$$
t^{\prime \prime}(s)=-s^{\prime}(t(s))^{-2} \cdot s^{\prime \prime}(t(s)) \cdot t^{\prime}(s)=\frac{-s^{\prime \prime}(t(s))}{s^{\prime}(t(s))^{3}} .
$$

Derivando a equação 5.3,

$$
s^{\prime \prime}(t)=s^{\prime}\left(t_{0}\right) \cdot e^{\int_{t_{0}}^{t} f(x) d x} \cdot f(t)=s^{\prime}(t) \cdot f(t),
$$

então

$$
t^{\prime \prime}(s)=\frac{-f(t(s))}{s^{\prime}(t(s))^{2}}
$$

Das equações 5.4 e 5.5,

$$
t^{\prime \prime}(s)+f(t(s)) \cdot t^{\prime}(s)^{2}=\frac{-f(t(s))}{s^{\prime}(t(s))^{2}}+f(t(s)) \cdot \frac{1}{s^{\prime}(t(s))^{2}}=0 .
$$

Portanto, $\tilde{\gamma}$ é uma geodésica.

O teorema 5.6 mostra uma relação entre as conexões de Levi-Civita de métricas conformes e é de se esperar então que exista uma relação entre geodésicas. E é o que veremos a seguir.

Teorema 5.9. Sejam $g$ e $g^{*}$ duas métricas conformes em $M$ tais que $g^{*}=\Lambda \cdot g$, com $\Lambda>0$. Então as pré-geodésicas tipo-luz de $g$ e $g^{*}$ coincidem. 
Demonstração. Só precisamos mostrar que se $\gamma$ é uma geodésica tipo-luz para $g$, então $\gamma$ é uma pré-geodésica tipo-luz para $g^{*}$, a recíproca é análoga. Embora a hipótese seja sobre pré-geodésicas, basta tomar a reparametrização geodésica e a demonstração segue.

Note que

$$
g^{*}\left(\gamma^{\prime}, \gamma^{\prime}\right)=0 \Leftrightarrow \Lambda \cdot g\left(\gamma^{\prime}, \gamma^{\prime}\right)=0 \Leftrightarrow g\left(\gamma^{\prime}, \gamma^{\prime}\right)=0 .
$$

Tomando o teorema 5.6 e supondo $X=Y=\gamma^{\prime}$, teremos então

$$
2 \Lambda \cdot \frac{D^{*}}{d t} \gamma^{\prime}=2 \Lambda \cdot \frac{D}{d t} \gamma^{\prime}+2 \gamma^{\prime}(\Lambda) \cdot \gamma^{\prime}-g\left(\gamma^{\prime}, \gamma^{\prime}\right) \nabla \Lambda
$$

Por hipótese, $\gamma$ é uma geodésica tipo-luz para $g$, então

$$
\frac{D}{d t} \gamma^{\prime}=0 \quad \text { e } \quad g\left(\gamma^{\prime}, \gamma^{\prime}\right)=0
$$

Logo, 5.6 fica escrito como

$$
\frac{D^{*}}{d t} \gamma^{\prime}=\frac{\gamma^{\prime}(\Lambda)}{\Lambda} \cdot \gamma^{\prime}
$$

Agora, basta aplicar o Lema 5.8 para $g^{*} \operatorname{com} f=\frac{\gamma^{\prime}(\Lambda)}{\Lambda}$ e portanto $\gamma$ é uma pré-geodésica tipo-luz para $g^{*}$.

\subsection{Independência de caráter de completude}

Quando falamos sobre completude de geodésicas em variedades semi-Riemannianas sempre precisamos levar em consideração o caráter causal das geodésicas.

Como vimos anteriormente, se $\gamma$ é uma geodésica em uma variedade semi-Riemanniana, então $g\left(\gamma^{\prime}(t), \gamma^{\prime}(t)\right)$ é constante para todo $t$. De modo que podemos classificar as geodésicas pelo caráter causal do campo $\gamma^{\prime}$. Por causa disso a completude de uma variedade precisa estar relacionda a um tipo de geodésica.

Definição 5.10. Seja $M$ uma variedade semi-Riemanniana. Dizemos que $M$ é geodesicamente completa tipo-espaço (respectivamente, tipo-tempo e tipo-luz) se toda geodésica tipo-espaço (respectivamente, tipo-tempo e tipo-luz) em M é completa.

Existem exemplos que mostram uma independência entre os três tipos de completude. Veremos nessa seção um exemplo de uma variedade completa tipo-tempo e tipo-luz e incompleta tipo-espaço. Esse exemplo pode ser encontrado em [Ger68].

Considere $\mathbb{L}^{2}$ com a métrica canônica $g=d x^{2}-d y^{2}$ e seja $g^{*}$ a métrica conforme definida por $g^{*}=e^{2 u(x, y)}\left(d x^{2}-d y^{2}\right)$, onde $u: \mathbb{R}^{2} \rightarrow \mathbb{R}$ é tal que

i. $\partial_{y} u(x, 0)=0$, para todo $x \in \mathbb{R}$;

ii. $u(x, y)=0$, se $|y|>1$;

iii. $\int_{-\infty}^{\infty} e^{u(x, 0)} d x<\infty$.

Note que estamos tomando nesse exemplo $\Lambda=e^{2 u}$. Pela Demonstração do Teorema 5.6 e usando que $\partial_{i}\left(e^{2 u}\right)=2 e^{2 u} \partial_{i} u$, temos:

$$
\nabla \Lambda=\sum_{i, j} g^{i j} \partial_{i} \Lambda \partial_{j}=\sum_{i, j} g^{i j} \partial_{i}\left(e^{2 u}\right) \partial_{j}=\sum_{i, j} 2 g^{i j} e^{2 u} \partial_{i} u \partial_{j}=2 \Lambda \nabla u
$$


Além disso, sendo $X \in \mathfrak{X}(M)$, segue que $X(\Lambda)=X\left(e^{2 u}\right)=2 \Lambda X(u)$. Então, a equação 5.1 pode ser reescrita como

$$
\begin{array}{r}
2 \Lambda \nabla_{X}^{*} Y=2 \Lambda \nabla_{X} Y+X(\Lambda) Y+Y(\Lambda) X-g(X, Y) \nabla \Lambda \\
=2 \Lambda \nabla_{X} Y+X(\Lambda) Y+Y(\Lambda) X-2 \Lambda g(X, Y) \nabla u \\
=2 \Lambda \nabla_{X} Y+2 \Lambda X(u) Y+2 \Lambda Y(u) X-2 \Lambda g(X, Y) \nabla u
\end{array}
$$

isto é,

$$
\nabla_{X}^{*} Y=\nabla_{X} Y+X(u) Y+Y(u) X-g(X, Y) \nabla u .
$$

Temos ainda que a equação 5.6 pode ser escrita como

$$
\frac{D^{*}}{d t} \gamma^{\prime}=\frac{D}{d t} \gamma^{\prime}+2 \gamma^{\prime}(u) \cdot \gamma^{\prime}-g\left(\gamma^{\prime}, \gamma^{\prime}\right) \nabla u
$$

Voltando ao exemplo anterior, podemos mostrar que $\left(\mathbb{L}^{2}, g^{*}\right)$ é incompleta tipo-espaço. De fato, tome a curva $\gamma(t)=(t, 0)$, com $t \in \mathbb{R}$, geodésica tipo-espaço para $g$. Então

$$
\gamma^{\prime}(u)=\sum_{i} \frac{d \gamma^{i}}{d t} \partial_{i} u=\partial_{x} u, \quad \nabla u=\sum_{i, j} g^{i j} \partial_{i} u \partial_{j}=\left(\partial_{x} u,-\partial_{y} u\right)
$$

e, da equação 5.7,

$$
\frac{D^{*}}{d t} \gamma^{\prime}=2\left(\partial_{x} u(t, 0), 0\right)-\left(\partial_{x} u(t, 0),-\partial_{y} u(t, 0)\right)=\left(\partial_{x} u(t, 0), \partial_{y} u(t, 0)\right) .
$$

Como $\partial_{y} u(x, 0)=0$, pela propriedade $i$. da função $u$, então

$$
\frac{D^{*}}{d t} \gamma^{\prime}(t)=\left(\partial_{x} u(t, 0), 0\right)=\partial_{x} u(t, 0) \cdot \gamma^{\prime}
$$

Tome $f(t)=\partial_{x} u(t, 0)$ e então, pelo Lema 5.8, temos que $\tilde{\gamma}$ é uma geodésica para $g^{*}$.

Note que, como vimos no Lema $5.8, \tilde{\gamma}(s)=(t(s), 0)$, onde

$$
s^{\prime}(t)=s^{\prime}\left(t_{0}\right) \cdot e^{\int_{t_{0}}^{t} f(x) d x}=C \cdot e^{u(t, 0)} .
$$

Dessa forma, note que

$$
s(t)-s\left(t_{0}\right)=\int_{t_{0}}^{t} s^{\prime}(x) d x=C \int_{t_{0}}^{t} e^{u(x, 0)} d x \leq C \int_{-\infty}^{\infty} e^{u(x, 0)} d x<\infty,
$$

onde a última desigualdade vem da propriedade iii. de $u$; então temos que $s$ é uma função limitada. Como $t$ e $s$ são funções inversas então o domínio de $t$ é um intervalo limitado, suponha $]-\xi, \xi[$. Além disso, como

$$
t^{\prime}(s)=\frac{1}{s^{\prime}(t(s))}
$$

então $\left|t^{\prime}(x)\right| \leq K$, para algum $K \in \mathbb{R}$ e para todo $\left.x \in\right]-\xi, \xi[$.

Assim, temos que $t$ é Lipschitziana e então, para qualquer $x \in]-\xi, \xi[$, temos que

$$
\lim _{y \rightarrow \xi}|t(x)-t(y)| \leq K \lim _{y \rightarrow \xi}|x-y|<2 K \xi
$$


Logo, $t$ é uma função limitada e não pode ser definida em $\mathbb{R}$. Portanto, $\left(\mathbb{R}^{2}, g^{*}\right)$ é incompleta tipo-espaço.

Suponha agora uma geodésica para $g^{*}$ do tipo causal, $\alpha(t)=(x(t), y(t))$, definida em um intervalo $I \subset \mathbb{R}$. Logo, $\alpha^{\prime}(t)=\left(x^{\prime}(t), y^{\prime}(t)\right)$ e

$$
0 \geq g^{*}\left(\alpha^{\prime}, \alpha^{\prime}\right)=e^{2 u\left(x^{\prime}, y^{\prime}\right)} \cdot g\left(\alpha^{\prime}, \alpha^{\prime}\right)=e^{2 u\left(x^{\prime}, y^{\prime}\right)} \cdot\left[\left(x^{\prime}\right)^{2}-\left(y^{\prime}\right)^{2}\right],
$$

então, como $e^{4 u(x, y)}>0$, para quaisquer $x, y \in \mathbb{R}$,

$$
\left(y^{\prime}\right)^{2} \geq\left(x^{\prime}\right)^{2} \Leftrightarrow\left|y^{\prime}(t)\right| \geq\left|x^{\prime}(t)\right|
$$

para $t \in I$. Além disso, $y^{\prime}(t) \neq 0$, para todo $t \in I$. Dessa forma, podemos reparametrizar $\alpha$ pela imagem de $y$. Seja $J=y(I)$ e tome a reparametrização $\tilde{\alpha}: J \rightarrow \mathbb{R}^{2}$ dada por $\tilde{\alpha}(y)=(x(y), y)$. Então $\tilde{\alpha}^{\prime}(y)=\left(x^{\prime}(y), 1\right)$ e, como $g^{*}=e^{2 u} g$,

$$
0 \geq g^{*}\left(\tilde{\alpha}^{\prime}, \tilde{\alpha}^{\prime}\right) \Rightarrow 0 \geq g\left(\tilde{\alpha}^{\prime}, \tilde{\alpha}^{\prime}\right)=\left(\frac{d x}{d y}\right)^{2}-1 \Rightarrow\left(\frac{d x}{d y}\right)^{2} \leq 1 \Rightarrow\left|\frac{d x}{d y}\right| \leq 1 .
$$

Para mostrar que $\left(\mathbb{R}^{2}, g^{*}\right)$ é completa tipo-causal, precisamos mostrar que $\alpha$ é completa. Observe primeiramente que a propriedade $i i$. da função $u$ definida acima delimita uma faixa fechada em $\mathbb{R}^{2}$ tal que

$$
g^{*}((x, y),(x, y))=g((x, y),(x, y)),
$$

para $|y|>1$. Ou seja, $g^{*}$ e $g$ coincidem fora da faixa. Nessa região, as geodésicas são retas, como visto no exemplo 2.60 , logo podem ser estendidas indefinidamente.

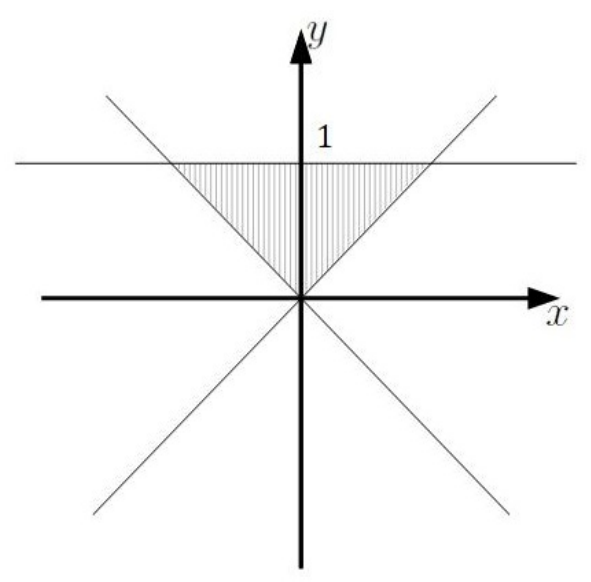

(a)

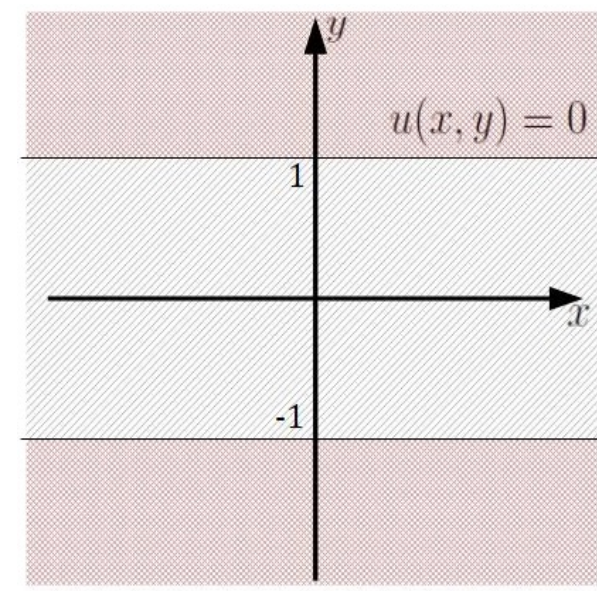

(b)

Figura 5.1: Local de definição dos vetores tangentes e representação da imagem da função u

Se $\alpha$ é uma geodésica iniciada fora da faixa, então é uma reta e pode ser estendida indefinidamente, ou seja, é completa. Se $\alpha$ inicia dentro da faixa, então note que saíra da faixa, pois, pela equação 5.9, os vetores tangentes estão direcionados para a reta $y=1$, como pode ser visto na Figura 5.3. Logo ao cruzar essa reta pode ser extendida indefinidamente.

A figura abaixo mostra uma situação em que a geodésica $\alpha$ inicia dentro da faixa, mas saíra da faixa em algum momento. 


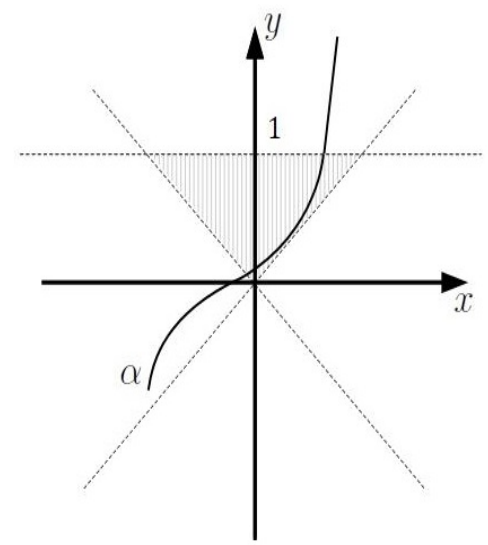

Figura 5.2: Esboço aproximado de uma geodésica $\alpha$

Ainda segundo [Ger68] é possível obter exemplos de variedades semi-Riemannianas nas seguintes situações

1. Completa tipo-tempo, incompleta tipo-espaço e tipo-luz;

2. Completa tipo-espaço, incompleta tipo-tempo e tipo-luz;

3. Completa tipo-luz, incompleta tipo-tempo e tipo-espaço;

4. Completa tipo-tempo e tipo-luz, incompleta tipo-espaço;

5. Completa tipo-espaço e tipo-luz, incompleta tipo-tempo.

De modo que, segundo ele, não é conhecido nenhum exemplo de uma variedade semiRiemanniana completa tipo-espaço e tipo-tempo e incompleta tipo-luz.

Quanto ao caso 5, considere a mesma métrica $g^{*}=e^{2 u} g$ e sendo $u$ uma função tal que

i. $\partial_{x} u(0, y)=0$, para todo $y \in \mathbb{R}$;

ii. $u(x, y)=0$, se $|x|>1$;

iii. $\int_{-\infty}^{\infty} e^{u(0, y)} d x<\infty$.

Dessa forma é possível mostrar de modo análogo ao caso anterior que $\left(\mathbb{L}^{2}, g^{*}\right)$ é completa tipo-espaço e tipo-luz e incompleta tipo-tempo.

Tomemos a curva $\gamma(t)=(0, t)$, geodésica tipo-tempo para $g$. Então

$$
\gamma^{\prime}(u)=\partial_{y} u \quad \text { e } \quad \nabla u=\left(\partial_{x} u,-\partial_{y} u\right) .
$$

Assim, pela equação 5.7,

$$
\frac{D^{*}}{d t} \gamma^{\prime}=2\left(0, \partial_{y} u(0, t)\right)+\left(\partial_{x} u(0, t),-\partial_{y} u(0, t)\right)=\left(\partial_{x} u(0, t), \partial_{y} u(0, t)\right) .
$$

Pela propriedade $i$. de $u, \partial_{x} u(0, t)=0$ e

$$
\frac{D^{*}}{d t} \gamma^{\prime}=\left(0, \partial_{y} u(0, t)\right)=\partial_{y} u(0, t) \cdot \gamma^{\prime} .
$$


Tomemos $f(t)=\partial_{y} u(0, t)$ e, pelo Lema $5.8, \tilde{\gamma}(s)=(0, t(s))$ é geodésica para $g^{*}$. Assim

$$
s^{\prime}(t)=s^{\prime}\left(t_{0}\right) \cdot e^{\int_{t_{0}}^{t} f(x) d x}=C \cdot e^{u(0, t)}
$$

e ainda

$$
s(x)=\int_{-\infty}^{\infty} s^{\prime}(x) d x=C \int_{-\infty}^{\infty} e^{u(0, x)} d x<\infty .
$$

O resto segue análogo ao caso anterior e temos que $t$ é limitada, portanto $\left(\mathbb{R}^{2}, g^{*}\right)$ é incompleta tipo-tempo.

O procedimento para mostrar a completude de $\alpha(t)=(x(t), y(t))$, geodésica não tipotempo para $g^{*}$, é análogo ao caso anterior, exceto que agora, sendo $\alpha^{\prime}(t)=\left(x^{\prime}(t), y^{\prime}(t)\right)$,

$$
\left(x^{\prime}\right)^{2}(t)-\left(y^{\prime}\right)^{2}(t) \geq 0 \Leftrightarrow\left|x^{\prime}(t)\right| \geq\left|y^{\prime}(t)\right|,
$$

para todo $t \in I$. E ao tomar a reparametrização $\tilde{\alpha}(x)=(x, y(x))$ teremos $\tilde{\alpha}^{\prime}(x)=\left(1, y^{\prime}(x)\right)$ e

$$
0 \leq 1-\left(\frac{d y}{d x}\right)^{2} \Rightarrow\left|\frac{d y}{d x}\right| \leq 1 .
$$

Assim, pelos cálculos acima e pela propriedade $i i$, teremos as seguintes situações

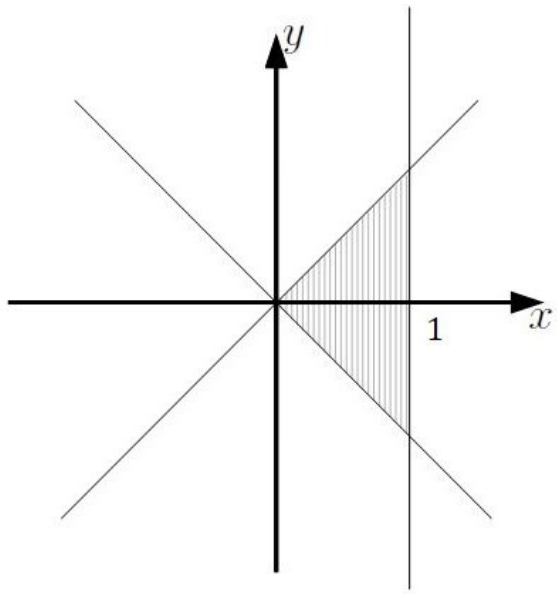

(a)

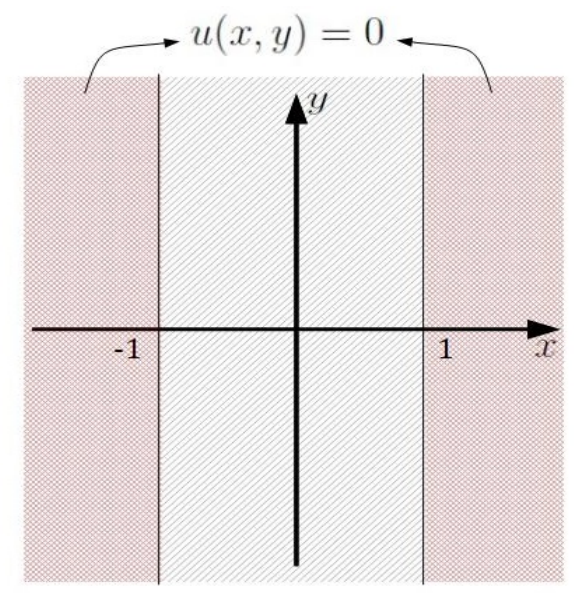

(b)

Figura 5.3: Local de definição dos vetores tangentes e representação da imagem da função u

De modo que a curva $\alpha$, se iniciada dentro da faixa, será do seguinte tipo

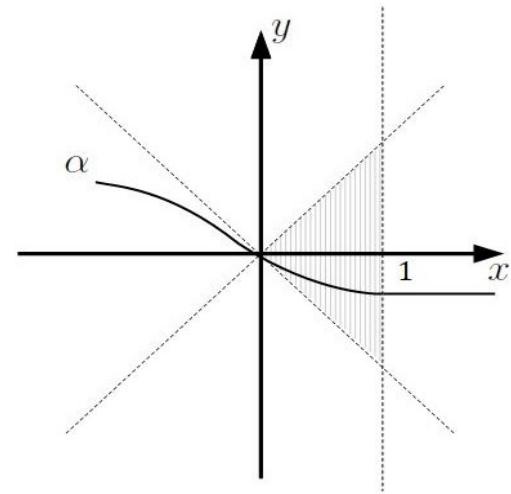

Figura 5.4: Esboço aproximado de uma geodésica $\alpha$ 
Exemplos mais genéricos e feitos de modo mais sistemático podem ser encontrados em [Kun63]. 


\section{Capítulo 6}

\section{Variedades compactas incompletas}

No Teorema 4.16 verificamos que para uma variedade semi-Riemanniana ser completa é necessário e suficiente que ela seja completa e homogênea e ainda no Capítulo 4 exibimos uma variedade homogênea e não compacta, e, por consequência, não completa. Nesse capítulo mostraremos uma variedade compacta, não homogênea e não completa.

\subsection{Família de métricas com campo de Killing}

Considere a seguinte métrica definida em $\mathbb{R}^{2}$

$$
g_{(x, y)}=E(x) d x^{2}+F(x)(d x \otimes d y+d y \otimes d x)-G(x) d y^{2},
$$

onde $E, F, G \in \mathcal{F}(\mathbb{R})$ satisfazem as seguinte condições:

i. $E(x) G(x)+F(x)^{2}>0$, para todo $x \in \mathbb{R}$;

ii. $E, G$ e $F$ são funções periódicas de período 1 .

Note que para pontos $p=\left(x_{0}, y_{0}\right),\left(x_{1}, y_{1}\right)$ e $\left(x_{2}, y_{2}\right)$ de $\mathbb{R}^{2}, g_{p}$ é calculado da seguinte forma

$$
g_{p}\left(\left(x_{1}, y_{1}\right),\left(x_{2}, y_{2}\right)\right)=E\left(x_{0}\right) x_{1} x_{2}+F\left(x_{0}\right)\left(x_{1} y_{2}+y_{1} x_{2}\right)-G\left(x_{0}\right) y_{1} y_{2},
$$

e agora podemos ver mais claramente que $g$ é uma métrica em $\mathbb{R}^{2}$. De fato, para todo $p \in M$,

- $g_{p}$ é bilinear. Sendo $\alpha, \beta \in \mathbb{R}$ quaisquer,

$$
\begin{array}{r}
g_{p}\left(\alpha\left(x_{1}, y_{1}\right)+\beta\left(x_{2}, y_{2}\right),(x, y)\right)=g_{p}\left(\left(\alpha x_{1}+\beta x_{2}, \alpha y_{1}+\beta y_{2}\right),(x, y)\right) \\
=E\left(x_{0}\right)\left(\alpha x_{1}+\beta x_{2}\right) x+F\left(x_{0}\right)\left[\left(\alpha x_{1}+\beta x_{2}\right) y+\left(\alpha y_{1}+\beta y_{2}\right) x\right]-G\left(x_{0}\right)\left(\alpha y_{1}+\beta y_{2}\right) y \\
=E\left(x_{0}\right)\left(\alpha x_{1} x+\beta x_{2} x\right)+F\left(x_{0}\right)\left[\alpha x_{1} y+\beta x_{2} y+\alpha y_{1} x+\beta y_{2} x\right]-G\left(x_{0}\right)\left(\alpha y_{1} y+\beta y_{2} y\right) \\
=\alpha\left[E\left(x_{0}\right) x_{1} x+F\left(x_{0}\right)\left(x_{1} y+y_{1} x\right)-G\left(x_{0}\right) y_{1} y\right] \\
+\beta\left[E\left(x_{0}\right) x_{2} x+F\left(x_{0}\right)\left(x_{2} y+y_{2} x\right)-G\left(x_{0}\right) y_{2} y\right] \\
=\alpha g_{p}\left(\left(x_{1}, y_{1}\right),(x, y)\right)+\beta g_{p}\left(\left(x_{2}, y_{2}\right),(x, y)\right)
\end{array}
$$




$$
\begin{array}{r}
g_{p}\left((x, y),\left(\alpha\left(x_{1}, y_{1}\right)+\beta\left(x_{2}, y_{2}\right)\right)\right)=g_{p}\left((x, y),\left(\alpha x_{1}+\beta x_{2}, \alpha y_{1}+\beta y_{2}\right)\right) \\
=E\left(x_{0}\right) x\left(\alpha x_{1}+\beta x_{2}\right)+F\left(x_{0}\right)\left[x\left(\alpha y_{1}+\beta y_{2}\right)+\left(\alpha x_{1}+\beta x_{2}\right) y\right]-G\left(x_{0}\right) y\left(\alpha y_{1}+\beta y_{2}\right) \\
=\alpha\left[E\left(x_{0}\right) x x_{1}+F\left(x_{0}\right)\left(x y_{1}+x_{1} y\right)-G\left(x_{0}\right) y y_{1}\right] \\
+\beta\left[E\left(x_{0}\right) x x_{2}+F\left(x_{0}\right)\left(x y_{2}+x_{2} y\right)\right]-G\left(x_{0}\right) y y_{2} \\
=\alpha g_{p}\left((x, y),\left(x_{1}, y_{1}\right)\right)+\beta g_{p}\left((x, y),\left(x_{2}, y_{2}\right)\right)
\end{array}
$$

- $g_{p}$ é simétrica, o que é fácil ver pela definição de $g_{p}$.

- $g_{p}$ é não degenerado. De fato, suponha que exista $(u, v)$ tal que $g_{p}((\bar{x}, \bar{y}),(x, y))=0$, para qualquer $(x, y) \in \mathbb{R}^{2}$. Logo,

$$
\left\{\begin{array}{l}
0=g_{p}((\bar{x}, \bar{y}),(1,0))=E\left(x_{0}\right) \bar{x}+F\left(x_{0}\right) \bar{y} \\
0=g_{p}((\bar{x}, \bar{y}),(0,1))=F\left(x_{0}\right) \bar{x}-G\left(x_{0}\right) \bar{y}
\end{array} .\right.
$$

Assim obtemos o seguinte sistema

$$
\left[\begin{array}{cc}
E\left(x_{0}\right) & F\left(x_{0}\right) \\
F\left(x_{0}\right) & -G\left(x_{0}\right)
\end{array}\right]\left[\begin{array}{l}
\bar{x} \\
\bar{y}
\end{array}\right]=\left[\begin{array}{l}
0 \\
0
\end{array}\right] .
$$

Então, pela propriedade $i$. das funções $E, F$ e $G, \bar{x}=\bar{y}=0$ e $g_{p}$ é não degenerada.

Das propriedades $i$. e $i i$. de $g_{p}$ podemos obter os seguintes resultados:

i. $g_{p}$ é uma métrica Lorentziana. De fato, como visto na Observação 2.32,

$$
(-1)^{\nu} \operatorname{det}\left[g_{p}\right]>0
$$

sendo $\left[g_{p}\right]$ a matriz de $g_{p}$, que indepente da base (como mostrado na Observação). Então, como, para $p=(x, y)$,

$$
\left[g_{p}\right]=\left[\begin{array}{cc}
E(x) & F(x) \\
F(x) & -G(x)
\end{array}\right]
$$

teremos que $\operatorname{det}\left[g_{p}\right]=-\left(E G+F^{2}\right)<0, \operatorname{logo}(-1)^{\nu}<0$ e $\nu=1$. Portanto, $g_{p}$ é métrica Lorentziana.

ii. $g_{p}$ é naturalmente induzida em um toro $\mathrm{T}^{2}=\mathbb{R}^{2} / \mathbb{Z}^{2}$. De fato isso vale pois as translações $T_{(1,0)}$ e $T_{(0,1)}$, nas direções dos eixos $x$ e $y$, respectivamente, são isometrias de $g_{p}$. Para mostrar isso vamos mostrar que os campos vetorias $\partial_{x}$ e $\partial_{y}$ são de Killing.

De fato, considere as curvas integrais em relação ao campo $\partial_{y}$ definidas como $\alpha: \mathbb{R} \rightarrow \mathbb{R}^{2}$, com

$$
\left\{\begin{array}{c}
\alpha(0)=p \\
\alpha^{\prime}(0)=\partial_{y}
\end{array}\right.
$$

Sendo $p=\left(x_{0}, y_{0}\right)$ e $\partial_{y}=(0,1)$, então $\alpha(t)=\left(x_{0}, y_{0}+t\right)$. Agora podemos definir o fluxo de $\partial_{y}$ em $\mathbb{R}^{2}, \psi: \mathbb{R} \times \mathbb{R}^{2} \rightarrow \mathbb{R}^{2}$ como

$$
\psi(t, p)=\alpha_{p}(t)
$$

com $p=(x, y)$ e $\alpha_{p}(t)=(x, y+t)$, uma curva integral de $\partial_{y}$. Dessa forma, podemos obter os estágios do fluxo $\psi_{t}: \mathbb{R}^{2} \rightarrow \mathbb{R}^{2}$. 
Logo,

$$
d \psi_{t}(x, y)=J\left[\psi_{t}\right](x, y)=\left[\begin{array}{ll}
1 & 0 \\
0 & 1
\end{array}\right] \cdot\left[\begin{array}{l}
x \\
y
\end{array}\right]=(x, y)
$$

então temos que

$$
\psi_{t}^{*}(g)\left(\left(x_{1}, y_{1}\right),\left(x_{2}, y_{2}\right)\right)=g\left(d \psi_{t}\left(x_{1}, y_{1}\right), d \psi_{t}\left(x_{2}, y_{2}\right)\right)=g\left(\left(x_{1}, y_{1}\right),\left(x_{2}, y_{2}\right)\right) .
$$

Logo, $\partial_{y}$ é de Killing e, pela Proposição $2.76, \psi_{t}$ é isometria, para qualquer $t \in \mathbb{R}$. Porém, note que $\psi_{t}$ é uma translação na segunda coordenada, então as translações em $y$ são isometrias.

De modo análogo é possível mostrar que translações em $x$ também são isometrias. E como $E, F$ e $G$ são funções de período 1 temos que

$$
g_{(x, y)}=g_{T_{(0, t)}(x, y)}=g_{T_{(t, 0)}(x, y)},
$$

para qualquer $t \in \mathbb{R}$. Portanto, a métrica $g_{p}$ pode ser associada ao toro $\mathrm{T}^{2}=\mathbb{R}^{2} / \mathbb{Z}^{2}$.

Observe que as métricas apresentadas acima formam uma família de métricas que podem ser induzidas no toro $\mathrm{T}^{2}$, variando dependendo da funções $E, F$ e $G$ tomadas. Definimos assim a família $\mathcal{G}$ de métricas do tipo 6.1.

Agora que vimos as propriedades básicas da família $\mathcal{G}$, seja $\gamma: I \subset \mathbb{R} \rightarrow \mathbb{R}^{2}$, dada por $\gamma(t)=(x(t), y(t))$, uma geodésica para $g \in \mathcal{G}$. Então

$$
\begin{array}{rcc}
g\left(\gamma^{\prime}(t), \gamma^{\prime}(t)\right)=\zeta & (\text { Proposição 2.61) } \\
g\left(\gamma^{\prime}(t), \partial_{y}\right)=\eta & (\text { Lema 2.78) }
\end{array}
$$

sendo $\zeta, \eta \in \mathbb{R}$. De $6.2 \mathrm{~b}$ temos que

$$
\eta=g_{\gamma(t)}\left(\left(x^{\prime}(t), y^{\prime}(t)\right),(0,1)\right)=F(x(t)) x^{\prime}(t)-G(x(t)) y^{\prime}(t) .
$$

E de 6.2a segue

$$
\zeta=g_{\gamma(t)}\left(\left(x^{\prime}(t), y^{\prime}(t)\right),\left(x^{\prime}(t), y^{\prime}(t)\right)\right)=E(x(t)) x^{\prime}(t)^{2}+2 F(x(t)) x^{\prime}(t) y^{\prime}(t)-G(x(t)) y^{\prime}(t)^{2},
$$

Logo,

$$
\zeta=E(x(t)) x^{\prime}(t)^{2}+y^{\prime}(t)\left[F(x(t)) x^{\prime}(t)+\eta\right] .
$$

Multiplicando por $G(x(t))$ a última igualdade e usando 6.3 segue

$$
\begin{array}{r}
G(x(t)) \zeta=G(x(t)) E(x(t)) x^{\prime}(t)^{2}+G(x(t)) y^{\prime}(t)\left[F(x(t)) x^{\prime}(t)+\eta\right] \\
=G(x(t)) E(x(t)) x^{\prime}(t)^{2}+\left[F(x(t)) x^{\prime}(t)-\eta\right]\left[F(x(t)) x^{\prime}(t)+\eta\right] \\
=G(x(t)) E(x(t)) x^{\prime}(t)^{2}+F(x(t))^{2} x^{\prime}(t)^{2}-\eta^{2} \\
=\left[G(x(t)) E(x(t))+F(x(t))^{2}\right] x^{\prime}(t)^{2}-\eta^{2} .
\end{array}
$$

Dessa forma

$$
x^{\prime}(t)^{2}=\frac{\eta^{2}+G(x(t)) \zeta}{E(x(t)) G(x(t))+F(x(t))^{2}} .
$$

Observe que qualquer geodésica satifaz as equações 6.3 e 6.4, entretanto nem todas as soluções dessas equações são geodésicas. Isso acontece pela perda de unicidade de soluções fixado condições iniciais. Tal perda de unicidade acontece quando 
i. $x^{\prime}=0$, para algum $t$; nesse caso reescrevemos as equações acima da seguinte forma

$$
\left\{\begin{array}{l}
6.4 \Rightarrow \eta^{2}+G\left(x\left(t_{0}\right)\right) \zeta=0 \\
6.3 \Rightarrow G\left(x\left(t_{0}\right)\right) y^{\prime}\left(t_{0}\right)+\eta=0
\end{array}\right.
$$

onde $t_{0}$ é tal que $x^{\prime}\left(t_{0}\right)=0$.

ii. $G(x)=0$; nesse caso reescrevemos as equações acima da seguinte forma

$$
\left\{\begin{array}{l}
6.4 \quad \Rightarrow \quad x^{\prime}(t)^{2}=\frac{\eta^{2}}{F(x(t))^{2}} \\
6.3 \quad \Rightarrow \quad F(x(t)) x^{\prime}(t)-\eta=0
\end{array}\right.
$$

Para corrigir o problema da perda de unicidade precisamos calcular as equações geodésicas 2.10 para a família $\mathcal{G}$.

Vamos calcular os símbolos de Christoffel. Sendo $\gamma(t)=(x(t), y(t))$ uma curva em T, primeiramente, note que

$$
\left[g_{\gamma(t)}\right]=\left[\begin{array}{cc}
E(x) & F(x) \\
F(x) & -G(x)
\end{array}\right] \quad \text { e } \quad\left[g_{\gamma(t)}\right]^{-1}=\frac{1}{A(x)}\left[\begin{array}{cc}
G(x) & F(x) \\
F(x) & -E(x)
\end{array}\right]
$$

onde $A(x)=\left(E G+F^{2}\right)(x)$. Dessa forma, os símbolos de Christoffel são determinados como segue

$$
\begin{array}{cc}
\Gamma_{11}^{1}(x)=\frac{G(x) E^{\prime}(x)+2 F(x) F^{\prime}(x)}{2\left[E(x) G(x)+F(x)^{2}\right]} & \Gamma_{11}^{2}(x)=\frac{F(x) E^{\prime}(x)-2 E(x) F^{\prime}(x)}{2\left[E(x) G(x)+F(x)^{2}\right]} \\
\Gamma_{12}^{1}(x)=\frac{-F(x) G^{\prime}(x)}{2\left[E(x) G(x)+F(x)^{2}\right]} & \Gamma_{12}^{2}(x)=\frac{E(x) G^{\prime}(x)}{2\left[E(x) G(x)+F(x)^{2}\right]} \\
\Gamma_{22}^{1}(x)=\frac{G(x) G^{\prime}(x)}{2\left[E(x) G(x)+F(x)^{2}\right]} & \Gamma_{22}^{2}(x)=\frac{F(x) G^{\prime}(x)}{2\left[E(x) G(x)+F(x)^{2}\right]}
\end{array}
$$

Omitindo as partículas $x$ e $t$ das funções $E, F$ e $G$ para fins de simplificação, então as equações geodésicas ficam escritas da seguinte forma

$$
\begin{aligned}
& A_{1}(t)=x^{\prime \prime}(t)+\left[\frac{G E^{\prime}+2 F F^{\prime}}{2\left(E G+F^{2}\right)}\right] x^{\prime}(t)^{2}-2\left[\frac{F G^{\prime}}{2\left(E G+F^{2}\right)}\right] x^{\prime}(t) y^{\prime}(t)+\left[\frac{G G^{\prime}}{2\left(E G+F^{2}\right)}\right] y^{\prime}(t)^{2} \\
& A_{2}(t)=y^{\prime \prime}(t)+\left[\frac{F E^{\prime}-2 E F^{\prime}}{2\left(E G+F^{2}\right)}\right] x^{\prime}(t)^{2}+2\left[\frac{E G^{\prime}}{2\left(E G+F^{2}\right)}\right] x^{\prime}(t) y^{\prime}(t)+\left[\frac{F G^{\prime}}{2\left(E G+F^{2}\right)}\right] y^{\prime}(t)^{2}
\end{aligned}
$$

O conjunto de equações 6.5 e as equações 6.4 e 6.3 fornecem tudo que é necessário para determinar as completudes e incompletudes do toro $\mathrm{T}^{2}$ munido de alguma métrica da família $\mathcal{G}$.

Faremos aqui um caso particular. Consideremos então a seguinte métrica

$$
g_{(x, y)}=d x \otimes d y+d y \otimes d x-\tau(x) d y^{2},
$$


onde $\tau: \mathbb{R} \rightarrow \mathbb{R}$ é uma função tal que:

$i$. é periódica de período 1 .

ii. $\tau(0)=0$.

iii. $\tau^{\prime}(0) \neq 0$.

Note que estamos tomando uma métrica de $\mathcal{G}$ e considerando $E \equiv 0, F \equiv 1, G=\tau$ e, além disso, algumas hipóteses extras sobre $\tau$. De modo geral, a métrica opera da seguinte forma em cada ponto $(x, y)$

$$
g_{(x, y)}\left(\left(x_{1}, y_{1}\right),\left(x_{2}, y_{2}\right)\right)=x_{1} \cdot y_{2}+y_{1} \cdot x_{2}-\tau(x) y_{1} \cdot y_{2} .
$$

Calculando as matrizes de $g$,

$$
\left[g_{(x, y)}\right]=\left[\begin{array}{cc}
0 & 1 \\
1 & -\tau(x)
\end{array}\right] \quad \text { e } \quad\left[g_{(x, y)}\right]^{-1}=\left[\begin{array}{cc}
\tau(x) & 1 \\
1 & 0
\end{array}\right],
$$

teremos os seguintes símbolos de Christoffel

$$
\begin{array}{lll}
\Gamma_{11}^{1}=0 & \Gamma_{11}^{2}=0 \\
\Gamma_{12}^{1}=\frac{1}{2} \tau^{\prime}(x) & \Gamma_{12}^{1}=0 \\
\Gamma_{22}^{1}=\frac{1}{2} \tau(x) \tau^{\prime}(x) & \Gamma_{22}^{2}=\frac{1}{2} \tau^{\prime}(x) .
\end{array}
$$

Consideremos uma curva $\gamma(t)=(x(t), y(t))$, com $t \in I \subset \mathbb{R}$, logo as equações geodésicas ficam escritas da seguinte forma

$$
\begin{gathered}
A_{1}(t)=x^{\prime \prime}(t)+\frac{1}{2} \tau^{\prime}(x(t)) x^{\prime}(t) y^{\prime}(t)+\frac{1}{2} \tau(x(t)) \tau^{\prime}(x(t)) y^{\prime}(t)^{2} \\
A_{2}(t)=y^{\prime \prime}(t)+\frac{1}{2} \tau^{\prime}(x(t)) y^{\prime}(t)^{2}
\end{gathered}
$$

Tomemos a curva $\gamma(t)=(0, y(t))$, com $t \in I \subset \mathbb{R}$, que parametriza o eixo $y$ em $\mathbb{R}^{2}$, assim

$$
g_{\gamma(t)}\left(\gamma^{\prime}(t), \gamma^{\prime}(t)\right)=g_{(0, y(t))}\left(\left(0, y^{\prime}(t)\right),\left(0, y^{\prime}(t)\right)=-\tau(0) y^{\prime}(t)^{2}=0,\right.
$$

onde a última igualdade vem da propriedade $i i$. Logo, os vetores $\gamma^{\prime}(t)$ são do tipo-luz para qualquer função $y: I \rightarrow \mathbb{R}$ e para qualquer $t \in I$. Teremos que $\gamma$ é geodésica se

$$
\begin{aligned}
& A_{1}(t)=\frac{1}{2} \tau(0) \tau^{\prime}(0) y^{\prime}(t)^{2}=0 \\
& A_{2}(t)=y^{\prime \prime}(t)+\frac{1}{2} \tau^{\prime}(0) y^{\prime}(t)^{2}=0
\end{aligned}
$$

e como, pela propriedade $i$. da função $\tau, \tau(0)=0$ temos $A_{1}(t)=0$, para todo $t \in \mathbb{R}$. Portanto, $\gamma$ é geodésica se

$$
y^{\prime \prime}(t)+\frac{1}{2} \tau^{\prime}(0) y^{\prime}(t)^{2}=0 .
$$


Pela propriedade $i i i$. da função $\tau, \tau^{\prime}(0) \neq 0$, então as soluções de 6.6 são do tipo

$$
y(t)=\frac{2}{\tau^{\prime}(0)} \ln \left(\tau^{\prime}(0) t+\alpha\right)+\beta,
$$

para $\alpha, \beta \in \mathbb{R}$. E agora é possível ver que $y$ não pode ser definida em todo $\mathbb{R}$, portanto $\gamma$, como definida acima, é uma geodésica tipo-luz incompleta.

Alguns resultados mais particulares podem ser demonstrados sem muita dificuldade, como veremos a seguir, porém um estudo mais completo sobre a incompletude do toro pode ser encontrada em [Caj97]. Entretanto já é possível observar que a familía de métricas $\mathcal{G}$ não é completa e, por extensão, pelo que fizemos no ínicio da seção, o toro $\mathrm{T}^{2}$ munido dessas métricas não é uma variedade completa.

As proposições a seguir podem ser encontradas em [O'N83], mas provaremos de modo distinto, usando as propriedades das métricas do tipo 6.1 e os resultados geométricos obtidos durante essa seção.

Proposição 6.1. Seja $\mathbb{R}^{2}$ munido de uma métrica $g \in \mathcal{G}$. Seja $\gamma(t)=\left(x_{0}, y(t)\right)$ uma curva em $\mathbb{R}^{2}$ com $x_{0} \in \mathbb{R}$ e $y^{\prime \prime} \equiv 0$. Então, $\gamma$ é uma geodésica se, e somente se, $G^{\prime} \equiv 0$.

Demonstração. Note que

$$
\gamma^{\prime}(t)=\left(0, y^{\prime}(t)\right)=y^{\prime}(t)(0,1)=y^{\prime}(t) \partial_{y} .
$$

Dessa forma,

$$
\frac{D}{d t} \gamma^{\prime}=\frac{D}{d t}\left[\gamma^{\prime}(t) \partial_{y}\right]=y^{\prime \prime}(t) \partial_{y}+y^{\prime}(t) \frac{D}{d t} \partial_{y}=y^{\prime \prime}(t) \partial_{y}+y^{\prime}(t) \nabla_{\gamma^{\prime}} \partial_{y}=y^{\prime}(t)^{2} \nabla_{\partial_{y}} \partial_{y} .
$$

Usando a equação 2.4 e os símbolos de Christoffel calculados anteriormente teremos que

$$
\begin{array}{r}
\nabla_{\partial_{y}} \partial_{y}=\sum_{i, k=1}^{2} X^{i}\left(\partial_{i} Y^{k}+\sum_{j=1}^{2} Y^{j} \Gamma_{i j}^{k}\right) \partial_{k}=X^{2}\left[Y^{2} \Gamma_{22}^{1} \partial_{x}+Y^{2} \Gamma_{22}^{2} \partial_{y}\right] \\
=\frac{1}{2} \cdot\left(\frac{G G^{\prime}}{E G+F^{2}} \partial_{x}+\frac{F G^{\prime}}{E G+F^{2}} \partial_{y}\right) .
\end{array}
$$

Então

$$
\frac{D}{d t} \gamma^{\prime}=\frac{y^{\prime}(t)^{2}}{2}\left(\frac{G G^{\prime}}{E G+F^{2}} \cdot \partial_{x}+\frac{F G^{\prime}}{E G+F^{2}} \cdot \partial_{y}\right)
$$

Suponha que $\gamma$ seja geodésica, então $\frac{D}{d t} \gamma^{\prime}=0$. Logo, como $y^{\prime} \neq 0$, devemos ter $G G^{\prime}=F G^{\prime}=0$, porém note que $G$ e $F$ nunca zeram simultaneamente, caso contrário, $E G+F^{2}=0$, o que contradiz propriedade i. de $g \in \mathcal{G}^{\prime}$. Portanto, $G^{\prime}\left(x_{0}\right)=0$.

Supondo agora que $G^{\prime}\left(x_{0}\right)=0$, é fácil ver que $\nabla_{\gamma^{\prime}} \gamma^{\prime}=0$ e, portanto, $\gamma$ é geodésica.

Proposição 6.2. Seja $\mathbb{R}^{2}$ munido de uma métrica $g \in \mathcal{G}$. Seja $\gamma(t)=\left(x(t), y_{0}\right)$ uma curva em $\mathbb{R}^{2}$ com $y_{0} \in \mathbb{R}$ e $x^{\prime \prime} \equiv 0$. Se $F \equiv 0$, então $\gamma$ é uma pré-geodésica.

Demonstração. Note que

$$
\gamma^{\prime}(t)=\left(x^{\prime}(t), 0\right)=x^{\prime}(t)(1,0)=x^{\prime}(t) \partial_{x} .
$$


De modo semelhante ao que fizemos na Proposição 6.1 calculamos

$$
\frac{D}{d t} \gamma^{\prime}=\frac{D}{d t}\left[x^{\prime}(t) \partial_{x}\right]=x^{\prime \prime}(t) \partial_{x}+x^{\prime}(t) \frac{D}{d t} \partial_{x}=x^{\prime}(t)^{2} \nabla_{\partial_{x}} \partial_{x}
$$

e ainda

$$
\nabla_{\partial_{x}} \partial_{x}=X^{1}\left(Y^{1} \Gamma_{11}^{1} \partial_{x}+Y^{1} \Gamma_{11}^{2} \partial_{y}\right)=\frac{1}{2}\left(\frac{G E^{\prime}+2 F F^{\prime}}{E G+F^{2}} \partial_{x}+\frac{F E^{\prime}-2 E F^{\prime}}{E G+F^{2}} \partial_{y}\right) .
$$

Como, por hipótese, $F \equiv 0$ então $F^{\prime} \equiv 0$, logo

$$
\frac{D}{d t} \gamma^{\prime}=\frac{x^{\prime}(t)^{2}}{2} \cdot \frac{E^{\prime}\left(x_{0}\right)}{E\left(x_{0}\right)} \partial_{x}=\frac{x^{\prime}(t)}{2} \cdot \frac{E^{\prime}\left(x_{0}\right)}{E\left(x_{0}\right)} \cdot \gamma^{\prime}
$$

Se $E^{\prime}\left(x_{0}\right)=0$, então $\frac{D}{d t} \gamma^{\prime}=0$ e $\gamma$ é geodésica, ao que segue o resultado. Se $E^{\prime}\left(x_{0}\right) \neq 0$, então note que

$$
\frac{D}{d t} \gamma^{\prime}=f(t) \cdot \gamma^{\prime}
$$

onde $f(t)=\frac{E^{\prime}\left(x_{0}\right)}{2 E\left(x_{0}\right)} x^{\prime}(t)$, e, pelo Lema 5.8, $\gamma$ é uma pré-geodésica.

\subsection{O toro de Clifton-Pohl}

Um outro exemplo clássico aplicado ao toro é o de Clifton-Pohl. Para construir esse toro vamos tomar primeiramente a seguinte métrica em $\mathbb{R}^{2}$

$$
g_{p}=f(x, y)(d x \otimes d y+d y \otimes d x),
$$

onde $f \in \mathcal{F}\left(\mathbb{R}^{2}\right)$ e $f>0$. Note que tal métrica é semelhante àquela feita na seção 6.1 ao tomarmos $E=G=0$, exceto que estamos tomando agora uma função de duas variáveis. Explicitamente, essa métrica é calculada da seguinte forma para $p=\left(x_{0}, y_{0}\right)$,

$$
g_{\left(x_{0}, y_{0}\right)}\left(\left(x_{1}, y_{1}\right),\left(x_{2}, y_{2}\right)\right)=f\left(x_{0}, y_{0}\right)\left(x_{1} y_{2}+x_{2} y_{1}\right)
$$

De fato, $g_{p}$ é uma métrica, pois

- $\operatorname{sejam}(x, y),\left(x_{1}, y_{1}\right),\left(x_{2}, y_{2}\right) \in \mathbb{R}^{2}$ e $\alpha \in \mathbb{R}$, então

$$
\begin{aligned}
& g_{p}\left(\alpha\left(x_{1}, y_{1}\right)+\left(x_{2}, y_{2}\right),(x, y)\right)=g_{p}\left(\left(\alpha x_{1}+x_{2}, \alpha y_{1}+y_{2}\right),(x, y)\right) \\
& =f\left(x_{0}, y_{0}\right)\left[\left(\alpha x_{1}+x_{2}\right) y+x\left(\alpha y_{1}+y_{2}\right)\right] \\
& =f\left(x_{0}, y_{0}\right)\left[\alpha\left(x_{1} y+x y_{1}\right)+\left(x_{2} y+x y_{2}\right)\right]=\alpha g_{p}\left(\left(x_{1}, y_{1}\right),(x, y)\right)+g_{p}\left(\left(x_{2}, y_{2}\right),(x, y)\right)
\end{aligned}
$$

$\mathrm{e}$

$$
\begin{aligned}
g_{p}\left((x, y), \alpha\left(x_{1}, y_{1}\right)+\left(x_{2}, y_{2}\right)\right)=g_{p}((x, y), & \left.\left(\alpha x_{1}+x_{2}, \alpha y_{1}+y_{2}\right)\right) \\
& =f\left(x_{0}, y_{0}\right)\left[x\left(\alpha y_{1}+y_{2}\right)+\left(\alpha x_{1}+x_{2}\right) y\right] \\
=f\left(x_{0}, y_{0}\right)\left[\alpha\left(x_{1} y+x y_{1}\right)+\left(x_{2} y+x y_{2}\right)\right]= & \alpha g_{p}\left((x, y),\left(x_{1}, y_{1}\right)\right)+g_{p}\left((x, y),\left(x_{2}, y_{2}\right)\right)
\end{aligned}
$$

- suponha que exista $(\bar{x}, \bar{y}) \in \mathbb{R}$ tal que $g_{p}((\bar{x}, \bar{y}),(x, y))=0$, para qualquer $(x, y) \in \mathbb{R}^{2}$. 
Então

$$
\begin{aligned}
& 0=g_{p}((\bar{x}, \bar{y}),(1,0))=f\left(x_{0}, y_{0}\right) \bar{y} \\
& 0=g_{p}((\bar{x}, \bar{y}),(0,1))=f\left(x_{0}, y_{0}\right) \bar{x}
\end{aligned}
$$

Logo, $(\bar{x}, \bar{y})=(0,0)$.

Portanto, $g_{p}$ é métrica.

$\mathrm{Na}$ forma matricial, podemos obter que

$$
\left[g_{p}\right]=\left[\begin{array}{cc}
0 & f \\
f & 0
\end{array}\right] \quad \text { e } \quad\left[g_{p}\right]^{-1}=\left[\begin{array}{cc}
0 & \frac{1}{f} \\
\frac{1}{f} & 0
\end{array}\right] .
$$

Obtemos assim os símbolos de Christoffel de $g_{p}$ ao longo de $\gamma$.

$$
\begin{array}{cc}
\Gamma_{11}^{1}(x, y)=\frac{1}{f(x, y)} \cdot \partial_{x} f(x, y) & \Gamma_{11}^{2}(x, y)=0 \\
\Gamma_{12}^{1}(x, y)=0 & \Gamma_{12}^{2}(x, y)=0 \\
\Gamma_{22}^{1}(x, y)=0 & \Gamma_{22}^{2}(x, y)=\frac{1}{f(x, y)} \cdot \partial_{y} f(x, y)
\end{array}
$$

Consideremos agora a variedade $\mathbb{R}^{2}-\{(0,0)\}$ e a métrica $g_{p}$ definida acima tomando $f(x, y)=\frac{1}{x^{2}+y^{2}}$. O toro de Clifton-Pohl é obtido de modo similar ao que foi feito no decorrer do capítulo 4 , tomando agora o grupo $G=\left\{\mu^{k} \mid k \in \mathbb{Z}\right\}$, sendo $\mu$ a homotetia

$$
\mu(x, y)=(2 x, 2 y) .
$$

De fato, $\mu$ é uma isometria, se $p=\left(x_{0}, y_{0}\right)$ e $\left(x_{1}, y_{1}\right),\left(x_{2}, y_{2}\right) \in \mathbb{R}^{2}-\{(0,0)\}$,

$$
\begin{aligned}
& g_{\mu(p)}\left(\mu\left(x_{1}, y_{1}\right), \mu\left(x_{2}, y_{2}\right)\right)= g_{\left(2 x_{0}, 2 y_{0}\right)}\left(\left(2 x_{1}, 2 y_{1}\right),\left(2 x_{2}, 2 y_{2}\right)\right)=\frac{4\left(x_{1} y_{2}+x_{2} y_{1}\right)}{\left(2 x_{0}\right)^{2}+\left(2 y_{0}\right)^{2}} \\
&=\frac{4\left(x_{1} y_{2}+x_{2} y_{1}\right)}{4\left(x_{0}^{2}+y_{0}^{2}\right)}=\frac{x_{1} y_{2}+x_{2} y_{1}}{x_{0}^{2}+y_{0}^{2}}=g_{p}\left(\left(x_{1}, y_{1}\right),\left(x_{2}, y_{2}\right)\right) .
\end{aligned}
$$

Definimos então o toro de Clifton-Pohl como sendo a variedade $\left(\mathrm{T}=\left(\mathbb{R}^{2}\right)^{*} / G, g_{p}\right)$. Que é obtido a partir do anel formado pelas circuferências de raios 1 e 2 e centro $(0,0)$, sendo que os pontos da fronteira são identificados pela ação do grupo $G$. 


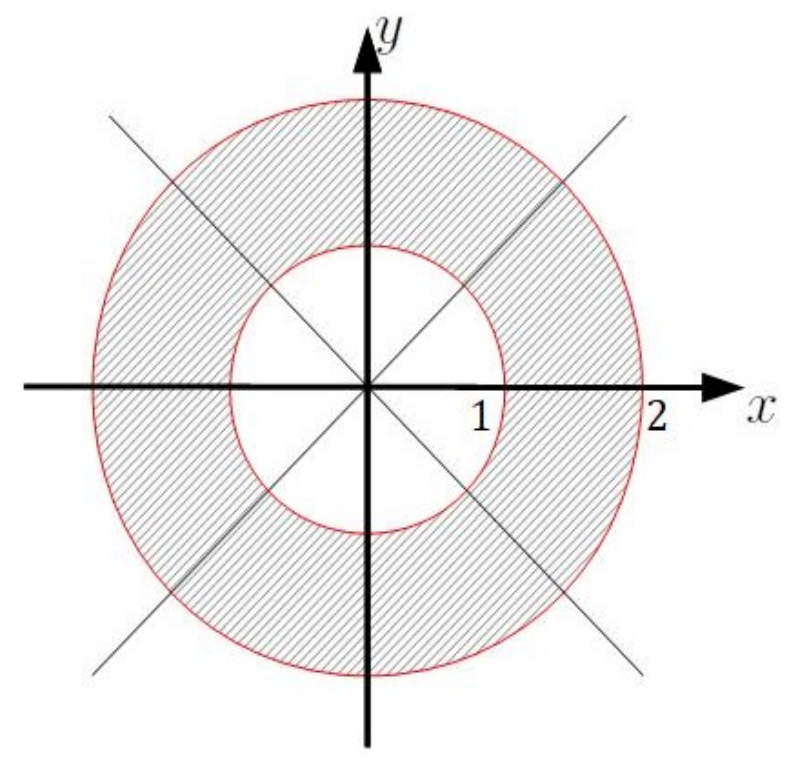

Figura 6.1: O toro de Clifton-Pohl ainda sem a identificação das fronteiras

Como estamos tomando a função $f(x, y)=\frac{1}{x^{2}+y^{2}}$, então temos que

$$
\partial_{x} f(x, y)=\frac{-2 x}{\left(x^{2}+y^{2}\right)^{2}} \quad \text { e } \quad \partial_{y} f(x, y)=\frac{-2 y}{\left(x^{2}+y^{2}\right)^{2}}
$$

e considerando a curva $\gamma: I \subset \mathbb{R} \rightarrow \mathrm{T}$, dada por $\gamma(t)=(x(t), y(t))$, temos que as equações geodésicas ficam determinadas como segue

$$
\begin{aligned}
& A_{1}(t)=x^{\prime \prime}(t)-\frac{2 x(t)}{x(t)^{2}+y(t)^{2}} \cdot x^{\prime}(t)^{2} \\
& A_{2}(t)=y^{\prime \prime}(t)-\frac{2 y(t)}{x(t)^{2}+y(t)^{2}} \cdot y^{\prime}(t)^{2}
\end{aligned}
$$

Para mostrar que $\left(\mathrm{T}, g_{p}\right)$ é incompleto basta tomar as geodésicas tipo-luz. Suponha que $\gamma(t)=(x(t), y(t))$ seja uma geodésica em $\mathrm{T}, \log \mathrm{O}$

$$
g_{\gamma(t)}\left(\gamma^{\prime}(t), \gamma^{\prime}(t)\right)=\frac{2 x^{\prime}(t) y^{\prime}(t)}{x(t)^{2}+y(t)^{2}}
$$

assim, é possível ver que $\gamma$ tipo-luz se, e somente se, $x^{\prime}(t)=0$ ou $y^{\prime}(t)=0$.

Vamos supor incialmente que $y^{\prime}(t)=0$, para todo $t \in I$. Nesse caso, $y(t)=y_{0}$, para alguma constante $y_{0} \in \mathbb{R}$ e $y_{0} \neq 0$. Como $\gamma$ é geodésica então

$$
\begin{gathered}
x^{\prime \prime}(t)-\frac{2 x(t)}{x(t)^{2}+y_{0}^{2}} \cdot x^{\prime}(t)^{2}=0 \\
y^{\prime \prime}(t)=0
\end{gathered}
$$

de onde tiramos que

$$
x(t)=y_{0} \tan \left(\rho y_{0}(\eta+t)\right),
$$

sendo $\rho, \eta \in \mathbb{R}$ constantes. Além disso, se $y_{0}=0$ então $x(t)=\frac{\eta}{\rho+t}$. 
De modo análogo, podemos considerar $x^{\prime}(t)=0$, para todo $t \in I$, em 6.8 , assim $x(t)=x_{0}$ e, se $x_{0} \neq 0$, temos

$$
\begin{gathered}
x^{\prime \prime}(t)=0 \\
y^{\prime \prime}(t)-\frac{2 y(t)}{x_{0}^{2}+y(t)^{2}} \cdot y^{\prime}(t)^{2}=0
\end{gathered}
$$

$\log 0$

$$
y(t)=x_{0} \tan \left(\rho x_{0}(\eta+t)\right)
$$

e, se $x_{0}=0, y(t)=\frac{\eta}{\rho+t}$.

A partir das equações 6.9 e 6.10 já é possível ver que $\left(\mathrm{T}^{2}, g_{p}\right)$ não é completo, pois é incompleto tipo-luz. De fato, dessas equações temos que as geodésicas em ambos os casos se escrevem como

$$
\left\{\begin{array}{cccc}
6.9 & \Rightarrow \gamma(t)=\left(y_{0} \tan \left(\rho y_{0}(\eta+t)\right), y_{0}\right) & \text { ou } & \gamma(t)=\left(\frac{\eta}{\rho+t}, 0\right) \\
6.10 & \Rightarrow \gamma(t)=\left(x_{0}, x_{0} \tan \left(\rho x_{0}(\eta+t)\right)\right) & \text { ou } & \gamma(t)=\left(0, \frac{\eta}{\rho+t}\right)
\end{array}\right.
$$

E agora podemos ver que como $\frac{\eta}{\rho+t}$ e tan $t$ são funções que não podem ser definidas para todo $\mathbb{R}$, então $\gamma$, sob nenhuma circunstância, pode ser estendida para $\mathbb{R}$ e, assim, ser completa.

Entretanto, se mais hipóteses forem feitas sobre a métrica Lorentziana genérica do toro (6.7) é então possível mostrar que T é completo em todos os caráteres causais. Deixamos [CR94] indicado como referência para esse resultado. 


\section{Referências Bibliográficas}

[BEE96] John K. Beem, Paul E. Ehrlich e Kevin L. Easley. Global Lorentzian geometry. CRC Press, 1996.

[Bie19] Rodney Josué Biezuner. Geometria Riemanniana. Disponível em http://150.164.25.15/ rodney/, 2016 (Acessado em 30/05/2019). Notas de Aula.

[Caj97] Miguel Sánchez Caja. Structure of Lorentzian tori with a Killing vector field. Transactions of the American Mathematical Society, 349(3):1063-1080, 1997.

[CC08] Anna Maria Candela e Miguel Sánchez Caja. Geodesics in semi-Riemannian manifolds: geometric properties and variational tools. Em Recent Developments in Pseudo-Riemannian Geometry, páginas 359-418. European Mathematical Society Publishing House, 2008.

[CR94] Yves Carrière e Luc Rozoy. Complétude des Métriques Lorentziennes de $\mathbb{T}^{2}$ et Difféomorphismes du Cercle. Boletim da Sociedade Brasileira de Matemática, 25(2):223-235, 1994.

[DI93] Della Duncan e Edwin Ihrig. Incomplete flat homogeneus geometries. Em Proceedings of Symposia in Pure Mathematics, volume 54.2, páginas 197-202. American Mathematical Society, 1993.

[Ger68] Robert Geroch. What is a Singularity in General Relativity? Annals of Physics, 48:526-540, 1968.

[HR31] Heinz Hopf e Willi Rinow. Ueber den Begriff der vollst ndigen differentialgeometrischen Fläche. Commentarii Mathematici Helvetici, 3(1):209-225, 1931.

[KN63] Shoshichi Kobayashi e Katsumi Nomizu. Foundations of Differential Geometry, volume I. Interscience Publishers, 1963.

[Kun63] Wolfgang Kundt. Note on the Completeness of Spacetimes. Zeitschrift für Physik, 172(5):488-489, 1963.

[Lim17] Elon Lages Lima. Curso de Análise, volume 1. IMPA, 2017.

[Mar73] Jerrold Marsden. On completeness of homogeneous pseudo-riemannian manifols. Indiana University Journal, 22:1065-1066, 1972-73.

[Mun00] James R. Munkres. Topology. Pearson, 2000.

[O’N83] Barrett O’Neill. Semi-Riemannian geometry. Academic Press, 1983.

[Pet06] Peter Petersen. Riemannian Geometry. Springer, 2006. 
[Ste12] Ian Stewart. Uma história da simetria na matemática. Zahar, 2012.

[VC10] Miguel Angel Javaloyes Victoria e Miguel Sánchez Caja. An introduction to Lorentzian Geometry and its Applications. RiMa, 2010. XVI Escola de Geometria Diferencial - USP. 